\title{
Calibrating Low-Frequency Spectrum of Moderate to Large Earthquakes using Stochastic Modelling Techniques
}

by

\section{Stephen Crane}

\author{
A Thesis submitted to \\ the Faculty of Graduate Studies and Research \\ in partial fulfilment of \\ the requirements for the degree of \\ Master of Science \\ in
}

Earth Sciences

Carleton University

Ottawa, Ontario, Canada

September 2011

Copyright (C)

2011 - Stephen Crane 
Library and Archives Canada

Published Heritage Branch

395 Wellington Street Ottawa ON K1A ON4 Canada
Bibliothèque et

Archives Canada

Direction du

Patrimoine de l'édition

395 , rue Wellington

Ottawa ON K1A ON4

Canada
Your file Votre référence

ISBN: 978-0-494-83177-9

Our file Notre référence

ISBN: $978-0-494-83177-9$
NOTICE:

The author has granted a nonexclusive license allowing Library and Archives Canada to reproduce, publish, archive, preserve, conserve, communicate to the public by telecommunication or on the Internet, loan, distribute and sell theses worldwide, for commercial or noncommercial purposes, in microform, paper, electronic and/or any other formats.

The author retains copyright ownership and moral rights in this thesis. Neither the thesis nor substantial extracts from it may be printed or otherwise reproduced without the author's permission.
AVIS:

L'auteur a accordé une licence non exclusive permettant à la Bibliothèque et Archives Canada de reproduire, publier, archiver, sauvegarder, conserver, transmettre au public par télécommunication ou par l'Internet, prêter, distribuer et vendre des thèses partout dans le monde, à des fins commerciales ou autres, sur support microforme, papier, électronique et/ou autres formats.

L'auteur conserve la propriété du droit d'auteur et des droits moraux qui protège cette thèse. $\mathrm{Ni}$ la thèse ni des extraits substantiels de celle-ci ne doivent être imprimés ou autrement reproduits sans son autorisation.
In compliance with the Canadian Privacy Act some supporting forms may have been removed from this thesis.

While these forms may be included in the document page count, their removal does not represent any loss of content from the thesis.
Conformément à la loi canadienne sur la protection de la vie privée, quelques formulaires secondaires ont été enlevés de cette thèse.

Bien que ces formulaires aient inclus dans la pagination, il n'y aura aucun contenu manquant.

\section{Canadä}




\begin{abstract}
Stochastic finite-fault modelling is a powerful tool used to simulate earthquake ground motions. It has proven useful for a number of different applications, those in the higher frequency range (about 1 to $5 \mathrm{~Hz}$ ) are of most interest to engineers. However it does have its shortcomings at the low-frequency range (below $1 \mathrm{~Hz}$ ) of ground motion spectrum, due to the underlying process of summing several smaller subfaults. Although the lowfrequency range has not been of much interest in the past, it is now commonly an integral part of earthquake engineering for larger structures. After calibrating the high-frequency spectrum for recorded earthquakes by adjusting the stress drop $(\Delta \sigma)$, the low-frequency spectrum is appropriately scaled using several different stochastic methods. One of these approaches introduces fractional stress drop as the low-frequency parameter, and the other is a low-frequency coefficient, introduced to EXSIM to create a scaling factor between high and low frequencies.
\end{abstract}




\section{Acknowledgments}

I would first like to thank my supervisor, Dr. Dariush Motazedian for his suggestions and guidance on this research topic. His support and encouragement helped to greatly advance my understanding of this subject. I would also like to thank Dr. David Boore, for his technical support and guidance with the SMSIM programs; the Pacific Earthquake Engineering Research Center, for making the Next Generation Attenuation Models database available; and, Dr. Giorgio Ranalli, Distinguished Research Professor at Carleton University, and Dr. Maurice Lamontagne of the Geological Survey of Canada and Adjunct Research Professor at Carleton University, for providing helpful knowledge of many related topics and processes.

Thank you to my fellow graduate students at Carleton University for their stimulating discussions and suggestions about this topic.

Lastly, I would like to extend my gratitude to my family and friends, especially Ashley, for their patience, support and interest in my studies. I am indebted for their constant encouragement.

This project is part of the Canadian Seismic Research Network (CSRN), which is funded by the Natural Sciences and Engineering Research Council of Canada. 


\section{Table of Contents}

Abstract

Acknowledgments iii

Table of Contents iv

List of Tables vii

List of Figures $\quad$ ix

1 Introduction 1

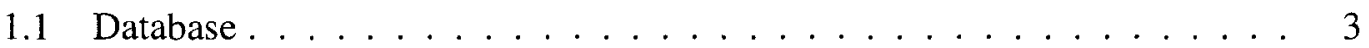

2 Earthquake Modelling 4

2.1 Stochastic Point-Source Modelling . . . . . . . . . . . . . . . 6

2.1 .1 SMSIM . . . . . . . . . . . . . 11

2.2 Stochastic Finite-Fault Modelling . . . . . . . . . . . . 12

2.2 .1 FINSIM . . . . . . . . . . . . . . . 13

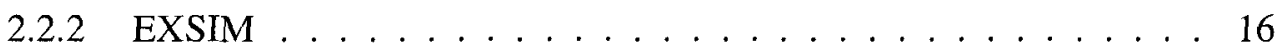

2.2 .3 EXSIM_Beta . . . . . . . . . . . . 18

3 Earthquake Parameters $\quad 20$

3.1 Source Parameters ....................... 21 
3.1 .1 Fault Geometry . . . . . . . . . . . . . . . 22

3.1 .2 Earthquake Size . . . . . . . . . . . . . 23

3.1 .3 Stress Drop . . . . . . . . . . . . . . . . . . . . . 24

3.1 .4 Rupture Process . . . . . . . . . . . . . . . 25

3.1 .5 Source Models . . . . . . . . . . . . . . . . . 26

3.2 Regional Path Effects . . . . . . . . . . . . . . . . 28

3.2 .1 Path Duration . . . . . . . . . . . . . . . . . . 29

3.2.2 Geometric Attenuation . . . . . . . . . . . . . . 30

3.2.3 Anelastic Attenuation and Quality Factor . . . . . . . . . . . 31

3.3 Site Effects . . . . . . . . . . . . . . . . . 32

3.4 Earthquake Spectrum . . . . . . . . . . . . . . 33

3.4.1 Boore's Low Frequency Taper . . . . . . . . . . . . . . . . 35

3.4.2 Motazedian's Low-Frequency Coefficient . . . . . . . . . . . 36

3.4 .3 Fractional Stress Drop . . . . . . . . . . . . . . 37

4 EXSIM Modifications

4.1 Several Hypocentres . . . . . . . . . . . . . . . . 42

4.2 Number of Effective Subfaults . . . . . . . . . . . . . . . . . 42

4.3 Fault Segments . . . . . . . . . . . . . . . . 43

4.4 Fractional Stress Drop . . . . . . . . . . . . . . . . . . 46

5 Stress Drop and Low-Frequency Values 53

5.1 General Residual Analysis . . . . . . . . . . . . . . . 54

5.2 Earthquake Specific Residual Analysis . . . . . . . . . . . . . . 62

6 Conclusion and Future Studies $\quad 70$

6.1 Suggested Future Studies . . . . . . . . . . . . . . . . 72

A Table of Western North America Parameters 
B Earthquake Specific Parameters

$\begin{array}{lll}\text { C } & \text { Table of Generic Earthquake Parameters } & 87\end{array}$

D FORTRAN Program for Calibrating Stress Drop and a Low Frequency Parameter 


\section{List of Tables}

1 A summary of the values contained in the constant of the source function. . 27

2 The reference source models for determining the overall source model contribution. ${ }^{*} \varepsilon$ is a scaling factor used for this model and is not the same value as fractional stress drop. ${ }^{\dagger}$ is the $\omega^{2}$ model . . . . . . . . . . . . 27

3 Corner frequencies and moment ratios. ${ }^{\dagger}$ is the $\omega^{2}$ model . . . . . . . . . 27

4 Generic crustal amplification from Atkinson and Boore (2006) with assumed shear wave velocity of $\geq 2 \mathrm{~km} / \mathrm{s} \ldots \ldots 33$

5 Frequency dependent amplification factors for NEHRP class C sites (Boore and Joyner, 1997) . . . . . . . . . . . . . . . . . . . . . 34

6 Frequency dependent amplification factors for NEHRP class D sites (Boore and Joyner, 1997) . . . . . . . . . . . . . . . . . . . . 34

7 Steps taken when the total residual sum is above the acceptable limit and the residuals above and below $1 \mathrm{~Hz}$ fall into each category . . . . . . . . 56

8 Steps taken when the total residual sum is below the acceptable limit and the residuals above and below $1 \mathrm{~Hz}$ fall into each category . . . . . . . . 56

9 Steps taken when the total residual sum is within the acceptable limit and the residuals above and below $1 \mathrm{~Hz}$ fall into each category . . . . . . . 5 56

10 Results for finding stress drop ( $\sigma$ ) in bars, low frequency coefficient (LFC) and fractional stress drop $(\varepsilon)$, along with the standard deviation of each $\left(\sigma_{s t d}, L F C_{s t d}\right.$, and $\left.\varepsilon_{s t d}\right)$ using a general residual analysis approach. . . . . . 58 
11 Results for finding stress drop $(\sigma)$ in bars, low frequency coefficient (LFC) and fractional stress drop $(\varepsilon)$ using an earthquake specific residual analysis approach. ........................ 64

12 A summary of the parameters used for simulating the earthquakes in Western North America. . . . . . . . . . . . . . . . . . . . . . . 75

13 A summary of the parameters used for simulating the Anza 02 earthquake. . 77

14 A summary of the parameters used for simulating the Coalinga 01 earthquake. 78

15 A summary of the parameters used for simulating the Coalinga 02 earthquake. 79

16 A summary of the parameters used for simulating the Hector Mine earthquake. . . . . . . . . . . . . . . . . . 80

17 A summary of the parameters used for simulating the Landers earthquake. . 81

18 A summary of the parameters used for simulating the Northridge 01 earth-

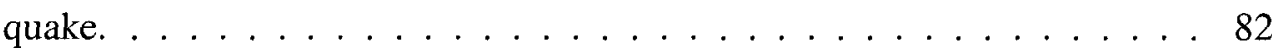

19 A summary of the parameters used for simulating the Northridge 04 earthquake. ....................... 83

20 A summary of the parameters used for simulating the Northridge 05 earthquake. ........................... 84

21 A summary of the parameters used for simulating the Northridge 06 earth-

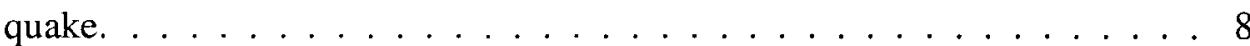

22 A summary of the parameters used for simulating the San Fernando earthquake.

23 The parameters used for simulating the generic earthquakes. The fault length and width were calculated using the magnitude relationships from Wells and Coppersmith (1994). . . . . . . . . . . . . . . . 88 


\section{List of Figures}

1 An illustration of point-source earthquake modelling. The fault plane is represented by a single point located on the fault. . . . . . . . . . 5

2 An illustration of finite-fault modelling. The fault surface is divided into smaller fault segments, and each subfault is treated as a point source. . . . . 6

3 The stochastic method for simulating an earthquake acceleration time series (Boore, 2003). The figures are from an actual simulation using parameters taken from Atkinson and Silva (2000) . . . . . . . . . . . . 8

4 Saragoni-Hart exponential window function and the variables controlling its shape $($ Boore, 2003) . . . . . . . . . . . . . . . . . . . 9

5 Two simulated acceleration time series using EXSIM. The input parameters were all the same except for the type of window applied. . . . . . . . . 11

6 Far-field energy of a fault with different subfault lengths using FINSIM (Motazedian and Atkinson, 2005). The event is an M7.0 earthquake at a distance of $333 \mathrm{~km}$, simulated using FINSIM (a static corner frequency approach) . . . . . . . . . . . . . . . . 15

7 Far-field energy of a fault with different subfault lengths using EXSIM (Motazedian and Atkinson, 2005). The event is an $M 7.0$ earthquake at a distance of $333 \mathrm{~km}$, simulated using the dynamic corner frequency approach. 17

8 An illustration of the fault geometry. . . . . . . . . . . . . . . . 22 
9 The effect on PSA values of adding Boore's taper to an earthquake simulation. The earthquake magnitude is $M 6.6$ at a distance of $55 \mathrm{~km}$ and a stress drop of 33 bars. Other parameters follow the generic earthquake parameters found in Table 23. . . . . . . . . . . . . . . . . . 36

10 The effect on PSA values when Motazedian's low-frequency coefficient $(L F C)$ is adjusted. The earthquake magnitude is $M 6.6$ at a distance of $55 \mathrm{~km}$ and a stress drop of 33bars. Other parameters follow the generic earthquake parameters found in Table 23. . . . . . . . . . . 38

11 The effect on PSA values when fractional stress drop $(F S D)$ is adjusted. The earthquake magnitude is $M 6.6$ at a distance of $55 \mathrm{~km}$ and a stress drop of 33 bars. Other parameters follow the generic earthquake parameters found in Table 23. . . . . . . . . . . . . . . . . . . 40

12 Map of the Landers region showing the location of the 1992 Landers earthquake. This earthquake occurred on three fault segments (Sommerville et al., 1997). . . . . . . . . . . . . . . . 4

13 Average residual of the 2002 Denali M7.9 earthquake using EXSIM and EXSIM_Beta modelling techniques. . . . . . . . . . . . . . . 46

14 A map showing the faults used for the EXSIM simulations along with the actual ruptured fault. The actual fault is projected from the USGS report on the Denali Park, Alaska earthquake of November 3, 2002. . . . . . . . . 47

15 A plot of $F(\varepsilon)$ versus frequency for different values of $\varepsilon \ldots \ldots$. . . . . . . 48

16 Simulated acceleration time series of $M 5$ and $M 7$ earthquakes at a distance of $100 \mathrm{~km}$ with $\varepsilon=1$ using a tapered boxcar window. . . . . . . . . . 50

17 Simulated acceleration time series of $M 5$ and $M 7$ earthquakes at a distance of $100 \mathrm{~km}$ with $\varepsilon=0.1$ using a tapered boxcar window. . . . . . . . . 50

18 Simulated acceleration time series of $M 5$ and $M 7$ earthquakes at a distance of $100 \mathrm{~km}$ with $\varepsilon=1.3$ using a tapered boxcar window. . . . . . . . . . 51 
19 Simulated Fourier acceleration spectrum of $M 5$ and $M 7$ earthquakes at a distance of $100 \mathrm{~km}$ for different values of $\varepsilon \ldots \ldots \ldots 1$

20 Simulated psuedo acceleration spectrum of $M 5$ and $M 7$ earthquakes at a distance of $100 \mathrm{~km}$ for different values of $\varepsilon \ldots \ldots \ldots 1$

21 Simulated psuedo velocity spectrum of $M 5$ and $M 7$ earthquakes at a distance of $100 \mathrm{~km}$ for different values of $\varepsilon \ldots \ldots \ldots 52$

22 Residuals for all earthquakes using the general residual analysis results for Boore's taper along with the average residual for each distance every $5 \mathrm{~km}$; and, the average residual at each frequency. . . . . . . . . . . 59

23 Residuals for all earthquakes using the general residual analysis results for Motazedian's low-frequency coefficient along with the average residual for each distance every $5 \mathrm{~km}$; and, the average residual at each frequency. . . . 60

24 Residuals for all earthquakes using the general residual analysis results for fractional stress drop along with the average residual for each distance every $5 \mathrm{~km}$; and, the average residual at each frequency. . . . . . . . . .

25 Average residuals at each frequency for each method using general residual analysis. . . . . . . . . . . . . . . . . 62

26 Residuals for the Northridge1 earthquake for Boore's taper, Motazedian's low-frequency coefficient and fractional stress drop using a general residual analysis approach. . ................. 63

27 Residuals for the Northridge1 earthquake for Boore's taper, Motazedian's low-frequency coefficient and fractional stress drop using an earthquake specific residual analysis approach with the residuals from the general residual approach included. . . . . . . . . . . . . . . . 63 
28 Residuals for all earthquakes using the earthquake specific residual analysis results for Boore's taper along with the average residual for each distance every $5 \mathrm{~km}$; and, the average residual at each frequency, including the average residual from the general residual analysis. . . . . . . . . . 66

29 Residuals for all earthquakes using the earthquake specific residual analysis results for Motazedian's low-frequency coefficient along with the average residual for each distance every $5 \mathrm{~km}$; and, the average residual at each frequency, including the average residual from the general residual analysis. 67

30 Residuals for all earthquakes using the earthquake specific residual analysis results for fractional stress drop along with the average residual for each distance every $5 \mathrm{~km}$; and, the average residual at each frequency, including the average residual from the general residual analysis. . . . . . . . . . 68

31 Average residuals at each frequency for each method using specific earthquake residual analysis. . . . . . . . . . . . . . . . . . . 69 


\section{Chapter 1}

\section{Introduction}

A large earthquake is a complicated process, where, after the build-up of stress over a long time the fault plane fails and repositions itself into a lower energy state. This can cause a sudden release of a large amount of stored energy, potentially damaging to nearby or even distant structures, depending on the size of the earthquake, the attenuation of the seismic waves with distance, and the local geological conditions. These effects have been consistently observed in the past in regions that have active seismic sources. With an ever increasing population and an increasing development of densely populated areas, the risk of negative impact as a result of an earthquake is constantly increasing. To reduce the risk arising from seismic activities, there are several areas of research, including seismicity rates in regions, possible locations and magnitudes of future earthquakes, and the level of ground motions as a result of these possible earthquakes.

The focus of this thesis is on the level of ground motions from possible earthquakes with an emphasis on scaling the low frequency portion of the spectrum. In order to correctly predict future ground motions, a model of the study region of western North America and earthquake parameters are tested with recorded motions from well studied past earthquakes. Different stochastic finite-fault modelling techniques, including strong motion simulation (SMSIM, Boore, 1996), finite-fault simulation (FINSIM, Beresnev and Atkinson, 1998a), and extended finite-fault simulation (EXSIM, Motazedian and Atkinson, 2005), are used 
to simulate the different earthquakes using data and known earthquake parameters from the Pacific Earthquake Engineering Research Center (PEER) Next Generation of GroundMotion Attenuation Models (NGA) database. The database contains psuedo-spectral acceleration (PSA) values in a wide range of frequencies from 0.1 to $100 \mathrm{~Hz}$, providing a good basis for spectral matching of both high and low frequencies.

One disadvantage of using a stochastic technique for modelling earthquakes is the poor performance of the low-frequency range. Using the data to the fullest extent, it is possible to compare the lower frequency portion of the spectrum to the simulated spectra. To develop a better agreement between the recorded and predicted motions in this range, a low-frequency parameter is included as an additional parameter to previous finite-fault modelling. Two different low-frequency parameters are included and tested to provide a better simulated low-frequency spectrum without drastically changing the rest of the spectrum.

Motazedian introduced a low-frequency coefficient in the new version of EXSIM, which is called EXSIM_Beta, as discussed in Chapter 2 (Section 2.2.3). This new version of EXSIM provides a low-frequency coefficient scaling factor. Fractional stress drop, as introduced by Brune (1970), is also added as an additional function to the EXSIM_Beta routine, providing an additional parameter to control the level of simulated low-frequency ground motions. The low-frequency spectrum is properly scaled after matching the highfrequency range, in order to provide a better match of other parameters, including stress drop, used in stochastic finite-fault modelling.

The stress parameter, $\Delta \sigma$, is an important parameter representing the high-frequency range of ground accelerations, which is the range of frequencies of the most importance for engineering purposes. The stress parameter has been measured or derived in a number of different studies (Brune, 1970; Boore et al., 1992; Atkinson and Boore, 1998; Beresnev and Atkinson, 1998a; Beresnev and Atkinson, 2002; Boore et al., 2010; Moghaddam et al., 2010), to provide some useful values for reproducing earthquake ground motions. These 
values have a wide range, but agree reasonably well when applied for their specific purpose; therefore, care must be taken in selecting an appropriate value when using the stress parameter for any purpose.

\subsection{Database}

The data used is contained in the PEER NGA database. This database contains 3,551 recordings of 175 different earthquakes, each having a range from a single site to 420 sites where values have been recorded. The recorded values include earthquake source parameters, site locations, site parameters, such as National Earthquake Hazard Reduction Program (NEHRP) building codes, $V_{s 30}$ (shear-wave velocity at a depth of 30 metres) values and geological information, distances from the earthquake to the site location, instrumentation types and filtering, peak ground acceleration, velocity and displacement values, and 5\%-damped psuedo-spectral accelerations at 105 different periods, ranging from 0.01 to 10 seconds. The listed ground motion values are computed as the $50 t h$ percent value of the geometric mean over the non-redundant 90 degrees range of rotation and represent the geometric average of two orthogonal horizontal components orientated randomly.

There are several recorded earthquakes used for the simulations, and the names are kept consistent with how they appear in the NGA database. Most of the parameters for these earthquakes used were taken directly from the NGA database, and some had to be inferred. Not all of the earthquake faults were completely described in the database, however they did have names of known California faults associated with them. These faults are all listed in the documentation for the United States National Seismic Hazard Maps (Frankel et al., 2002), and those defined faults were used. 


\section{Chapter 2}

\section{Earthquake Modelling}

There are many different methods and approaches for modelling any particular earthquake, including deterministic models, stochastic point-source models, stochastic finite-fault models and hybrid models. These models all vary in complexity and precision; most of which have different limitations and applications to earthquakes. Each type of modelling has its own purpose, whether it is a simple model that can be calculated quickly, such as pointsource modelling, or more complex models that may take significantly longer to calculate, but are more accurate.

Deterministic methods (Boatwright, 1982; Kamae et al., 1998; Frankel, 2009) use a wide range of known source, path and site parameters with developed mathematical equations to determine the effects of an earthquake. The equations are derived using statistical methods on a large dataset of recorded earthquakes within different regions. The equations can map earthquake effects from a wide variety of source parameters and can provide reasonable mathematical relations from one parameter to another. These models are generally region specific, but can be adapted to other areas for predictive purposes.

An inconvenient limitation, however, occurs with the dataset, which only gives precise results if there is a wide range of data available. This is difficult in most areas as a long time frame, in the order of decades or even hundreds of years, and a large number of seismometer stations are needed to record earthquakes of all magnitudes at a wide variety 


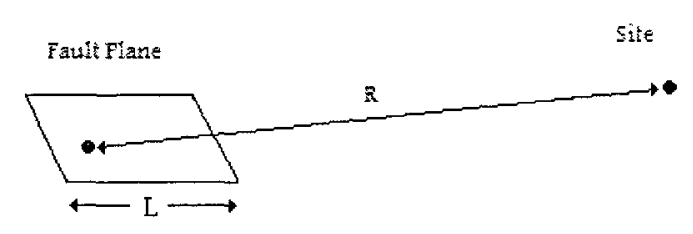

Figure 1: An illustration of point-source earthquake modelling. The fault plane is represented by a single point located on the fault.

of distances.

Stochastic methods incorporate the randomness of ground motion along with earthquake characteristics to simulate possible effects. Similar to deterministic methods, they use simplified mathematical equations to predict the resulting effects of an earthquake, but some random components are added to simulate the complexity of earthquake faulting. There are several different stochastic methods that apply some addition of a random phase at a certain time in the calculation. The stochastic method used here follows Boore, (1983 and 2003), which is based on the works of Hanks and McGuire for the incorporation of the random behaviour of high-frequency motions (Hanks, 1979; McGuire and Hanks, 1980; Hanks and McGuire, 1981; Boore, 2003).

Point-source modelling of an earthquake reduces the entire fault plane into a single point, which is often taken as the location where the most energy is released. This is illustrated in Figure 1, where the fault surface is represented as a single point. Approximating an earthquake as a point greatly reduces the complexity of the source, as there is a reduced amount of geometry and no planar features. Even though earthquake fault planes can be large, on the order of $10 \mathrm{~s}$ or $100 \mathrm{~s}$ of kilometres, this approximation holds well if the site is a large distance away or the earthquake is small. The path length should be much larger than the fault length.

Finite-fault modelling uses the same process as point source models; however, it breaks the fault plane into smaller subfaults. Each of the individual subfaults acts as a separate 


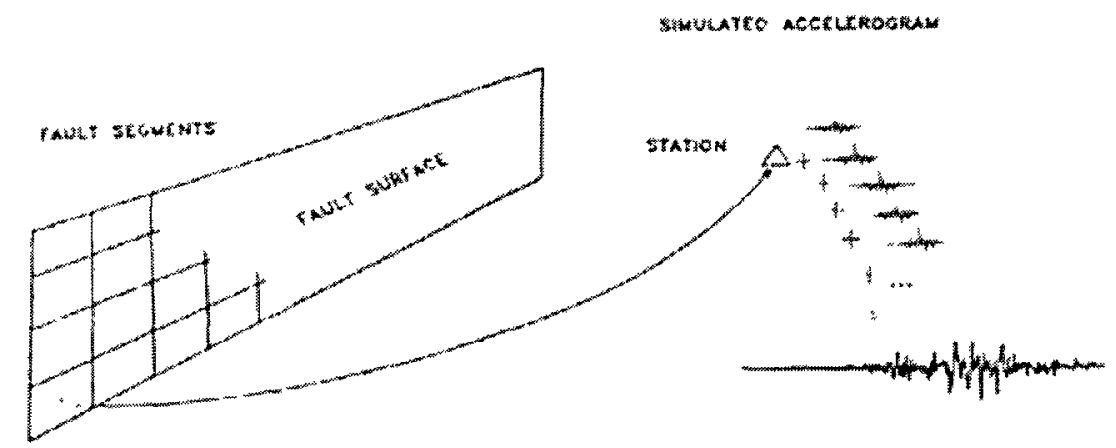

Figure 2: An illustration of finite-fault modelling. The fault surface is divided into smaller fault segments, and each subfault is treated as a point source.

point source; and, the contribution of each subfault, along with an appropriate time delay, is summed to produce the larger desired effects. This type of modelling is illustrated in Figure 2. The complexity of a finite-fault source is a bit more than a point source; however, the accuracy and time required for calculations increases as the total number of subfaults increases.

\subsection{Stochastic Point-Source Modelling}

A simple and powerful tool to simulate earthquake ground motions is a method called stochastic point-source modelling. It essentially combines the simplicity of point-source earthquake model along with the process of stochastic modelling, in order to generate an earthquake time series at a location far from the source. The method used here is the foundation of the earthquake modelling, and is based on the works of Boore (1983 and 2003).

The stochastic method has a very distinct order of process that affects the results if not followed correctly (Safak and Boore, 1988). Figure 3 shows the illustrated steps in order of application. First, a random noise is created over a desired length of time (Figure 3a). A Gaussian white noise is preferred, but other uniformly distributed noises can be used 
instead.

A window is then applied to the noise to simulate the shape of the envelope of the desired earthquake time series (Figure 3b). A Saragoni-Hart window is the best description of a final earthquake time series envelope, but other windows, such as a boxcar with tapered edges, can also be used. The noise is then transformed into the frequency domain (Figure $3 \mathrm{c}$ ) and normalized by the square root of the mean squared amplitude spectrum (Figure $3 \mathrm{~d}$ ).

The frequency spectrum is multiplied by an average desired spectral spectrum described by the target earthquake (Figure 3e). The frequency spectrum is then transformed back into the time domain. This creates a randomly simulated earthquake time series that should contain a frequency spectrum similar to one that is expected from an earthquake of the same size and magnitude (Figure $3 \mathrm{f}$ ).

Most of the steps (i.e. 1, 3, 4 and 6) are purely mathematical, and no prior knowledge of the target earthquake is needed. Step 2, windowing of the random noise, does have a small dependence on the target earthquake, as size of the window is dependent on the desired duration of the earthquake. The shape and parameters of the applied window depends on which window is used. Step 5, multiplication of a normalized frequency spectrum by a target frequency spectrum, contains most of the physics of both the earthquake process and wave propagation. It is usually put in the form of simple equations that incorporate the parameters affecting the earthquake ground motions.

The Saragoni-Hart window has an exponential shape, which is a good representation of a propagating earthquake envelope. The window, following the notation of Boore (2003), is:

$$
w\left(t ; \varepsilon, \eta, t_{\eta}\right)=a\left(\frac{t}{t_{\eta}}\right)^{b} \exp \left(-c\left(\frac{t}{t_{\eta}}\right)\right)
$$

Figure 4 shows the shape of the window function along with the different variables that control the shape of the function. Parameters a, b, and $\mathrm{c}$ are determined in a way that $w(t)$ has a unity peak value when $t=\varepsilon \times t_{\eta}$, and $w(t)=\eta$ when $t=t_{\eta}$. The equations for $\mathrm{a}, \mathrm{b}$, 


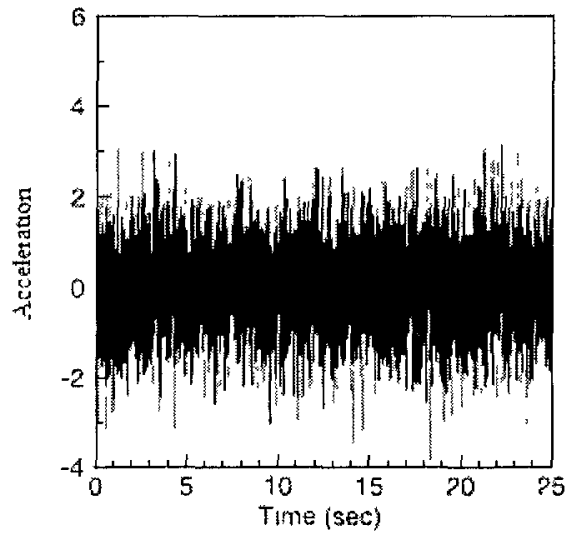

a) Step 1: Create noise

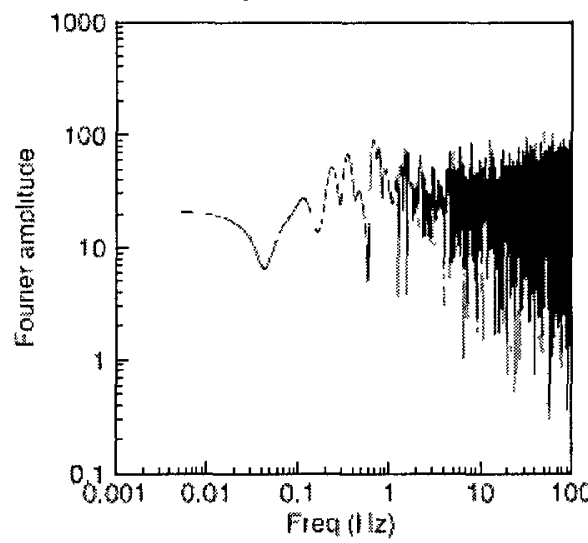

c) Step 3. Transform in frequency domain

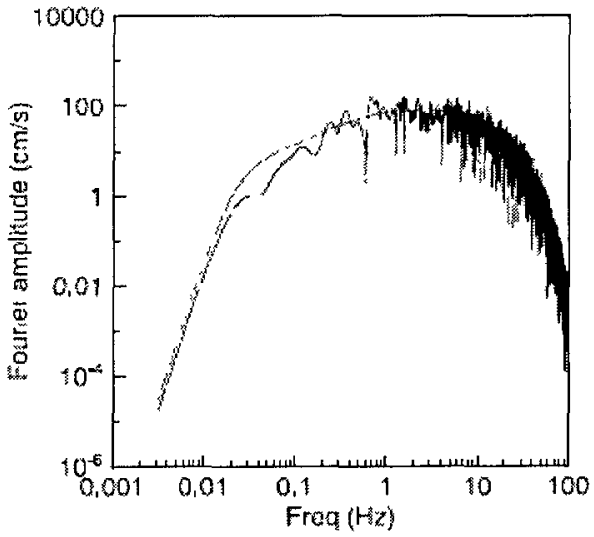

e) Step 5: Multiply by target spectrum

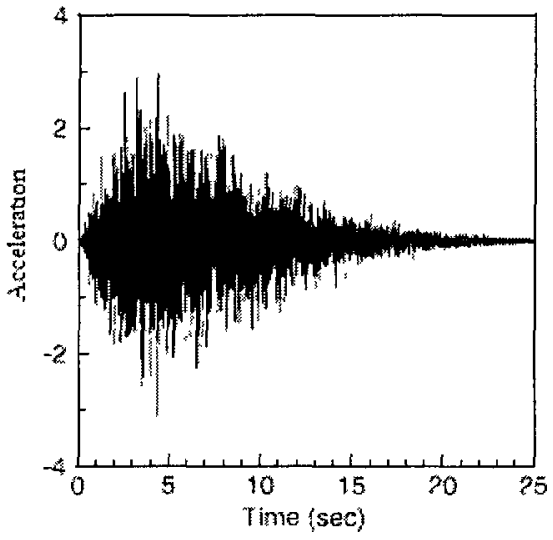

b) Step 2: Window noise

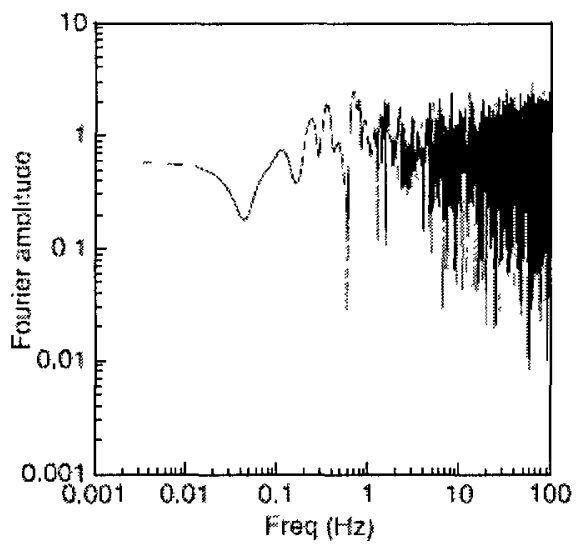

d) Step 4: Normalize spectrum

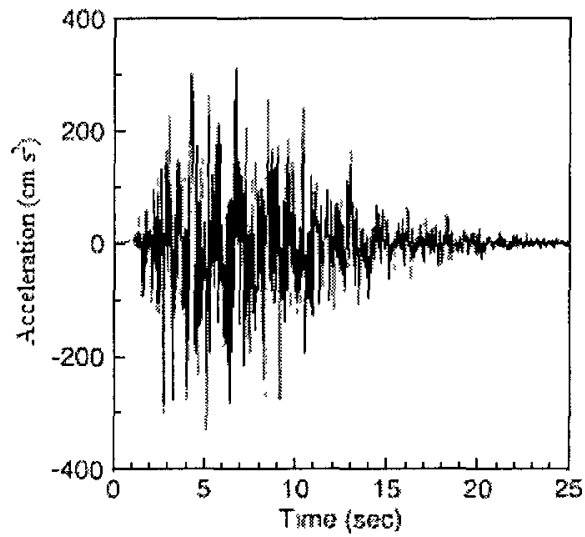

f) Step 6: Transform back into time domain

Figure 3: The stochastic method for simulating an earthquake acceleration time series (Boore, 2003). The figures are from an actual simulation using parameters taken from Atkinson and Silva (2000). 


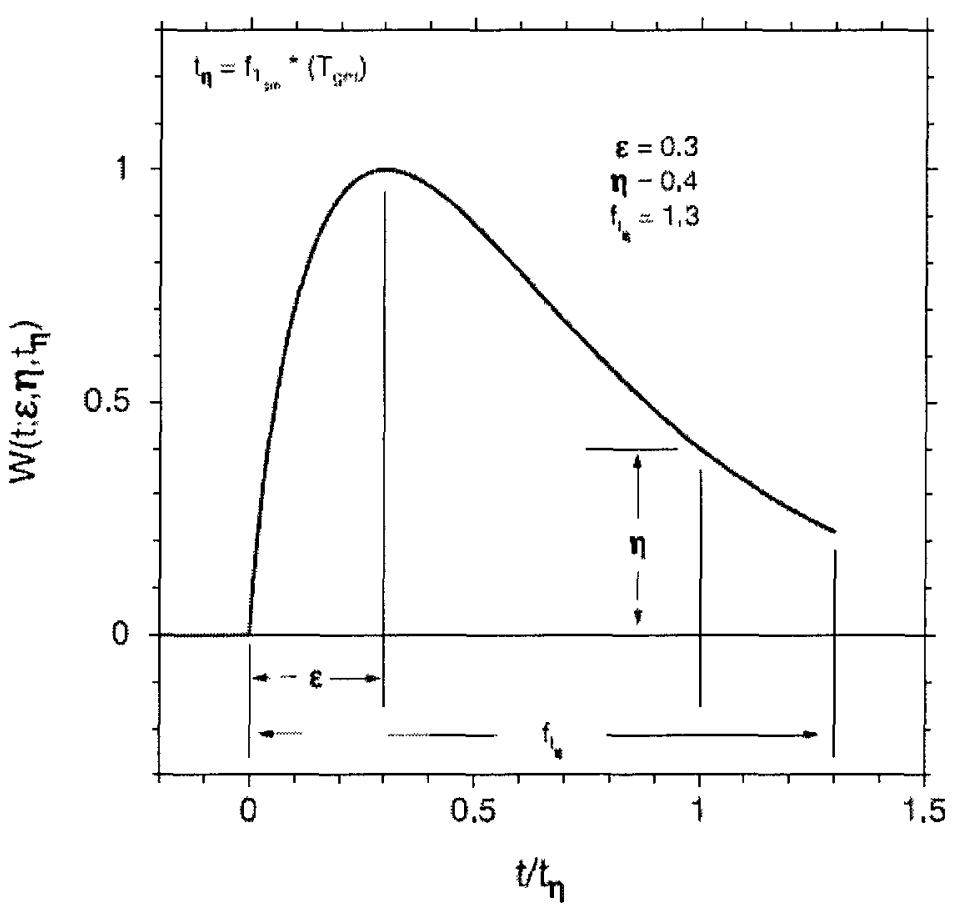

Figure 4: Saragoni-Hart exponential window function and the variables controlling its shape (Boore, 2003). 
and $\mathrm{c}$ are:

$$
\begin{gathered}
b=\frac{-(\varepsilon \ln \eta)}{1+\varepsilon(\ln \varepsilon-1)} \\
c=\frac{b}{\varepsilon} \\
a=\left(\frac{\exp (1)}{\varepsilon}\right)^{b}
\end{gathered}
$$

The time $t_{\eta}$ is given by

$$
t_{\eta}=f_{T_{g m}} \times T_{g m}
$$

where $T_{g m}$ is the duration of ground motions. This is determined as the time it takes to reach roughly $75 \%$ of the peak motion, from the start of the ground motions.

Using $f_{T_{g m}}=2.0$ gives a good comparison between the response spectra calculated using the Saragoni-Hart window and the tapered boxcar window (Boore, 2003). The frequency content of both windows is comparable. The main advantage of the Saragoni-Hart window is its aesthetic look, which is more similar to a recorded earthquake time series. Figure 5 shows two acceleration time series simulated with the same input, except one was windowed with a boxcar taper (Figure 5a), and the other was windowed with the SaragoniHart window (Figure 5b).

The essence of this method is the target frequency spectrum of an earthquake, which is the most important determining factor of the resulting earthquake time series. A lot of research efforts (Boore, 1983; Atkinson and Boore 1995; Haddon 1996; Beresnev and Atkinson, 1998a; Motazedian and Atkinson 2005) have been put into determining the best and simplest equations that describe the frequency content of an earthquake. The target spectrum can be represented by a simple equation:

$$
A(f)=E\left(M_{0}, f\right) P(R, f) S(f)
$$

where $M_{0}$ is the seismic moment. The frequency dependent acceleration spectrum, $A(f)$, 

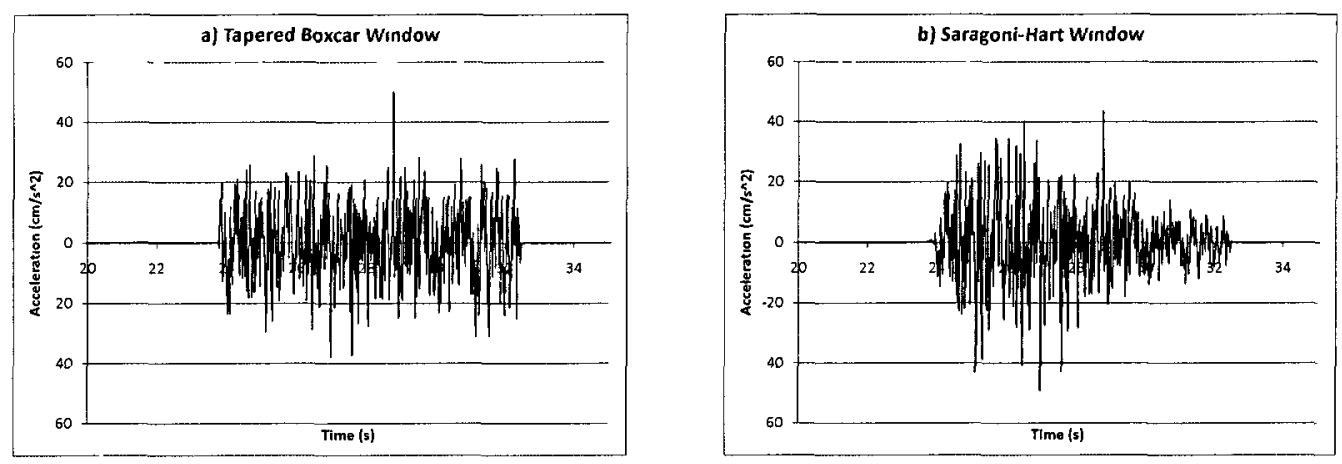

Figure 5: Two simulated acceleration time series using EXSIM. The input parameters were all the same except for the type of window applied.

is split up into several contributing factors. The source function, $E\left(M_{0}, f\right)$, contains the parameters for the actual earthquake. The path function, $P(R, f)$, describe the effects of the envelope propagating through a medium. The site function, $S(f)$, contains the response of the location where the earthquake is felt. All these functions are explained in Chapter 3.

\subsubsection{SMSIM}

The SMSIM set of programs were created by Boore (1996) and are called strong motion simulation (or stochastic model simulation). These were designed on the basis of using stochastic point-source modelling to simulate an earthquake time series. The programs use either random vibration or time-domain simulations, with most of the input and output parameter files being similar. The time-domain simulation programs are used in this research because they are the most similar to EXSIM and the other programs which are used. Timedomain simulations do not contain the same assumptions made in random vibration theory (Boore, 1996).

The SMSIM programs require the input file to contain the model parameters and some SMSIM specific parameters being used for the simulation. These include the density, shear 
wave velocity, partition factor, radiation pattern, free surface factor, source number, spectral shape controlling factors, stress drop and other scaling spectral amplitude parameters, geometrical spreading parameters, path attenuation, source and path duration, site amplification, choice of high-frequency filter and corresponding value, optional low-cut filter, the window type and corresponding window parameters, time-domain parameters including time spacing and the initial time shift, the number of simulations to sum, and the type of random noise created. Most of these parameters are discussed in more detail in Chapter 3. The option for source type refers to using either a single-corner frequency or one of several double corner-frequency methods (Atkinson and Boore, 1995; Haddon 1996).

The driver programs used here are called GM_TD_DRVR (ground motion timedomain driver) and FAS_TD_DRVR (Fourier amplitude spectrum time-domain driver). The ground-motion driver computes peak velocity, peak acceleration, Arias intensity, and response-spectral amplitudes. The Fourier amplitude spectrum driver calculates the average Fourier spectral amplitudes by generating a number of time series, computing the Fourier spectra of each one, and determining the average of the spectra.

These two drivers were used to create response and Fourier amplitude spectra comparable to those created by other programs, using the same input parameters and similar calculations of the target frequency spectrum for a number of earthquakes (Boore, 1983; Joyner, 1984; Atkinson and Boore, 1998; Atkinson and Silva, 2000).

\subsection{Stochastic Finite-Fault Modelling}

Stochastic finite-fault modelling uses and extends many of the same concepts of stochastic point-source modelling. The earthquake fault plane is divided into $N$ smaller subfaults, whose size can be anywhere from a one kilometre to several kilometres in length and width. Each subfault is treated as a single earthquake and a single time series is generated using a stochastic point source technique. The final time series is calculated by summing the 
individual contributions of each subfault with the addition of an appropriate time delay.

$$
A(t)=\sum_{t=0}^{n l} \sum_{j=0}^{n w} a_{l j}\left(t+\Delta t_{l \jmath}\right)
$$

$A(t)$ is the final time series, $n l$ and $n w$ are the total number of subfaults along the length and width, $a_{l j}(t)$ is the time series for each $i$ th and $j$ th subfault, and $\Delta t_{l j}$ is the time delay for the corresponding subfault.

The seismic moment for each subfault is determined from the ratio of the subfault to the main fault. If all the subfaults are identical then

$$
M_{0 l \jmath}=\frac{M_{0}}{N}
$$

where $M_{0 l j}$ is the seismic moment of a subfault. If the subfaults are not identical, then the seismic moment can be determined from an array of slip weights, $S_{l j}$, by:

$$
M_{0 l \jmath}=\frac{M_{0} S_{l j}}{\sum_{l=1}^{n l} \sum_{k=1}^{n w} S_{k l}}
$$

The summation of the slip weights array ensures the sum of the subfault seismic moments is equal to the total seismic moment.

\subsubsection{FINSIM}

FINSIM, a FORTRAN program, simulates a stochastic ground acceleration series using finite-fault modelling (Beresnev and Atkinson 1998). This program determines an individual seismic moment $\left(m_{0}\right)$ by:

$$
m_{0}=\Delta \sigma \Delta l^{3}
$$

$\Delta \sigma$ is the stress drop and $\Delta l$ is the length of a subfault. For simplicity, it is assumed that the subfault length is equal to the subfault width $(\Delta l=\Delta w)$. The corner frequency is determined 
by

$$
f_{0}=\frac{\left(\frac{\eta z}{\pi}\right) \beta}{\Delta l}
$$

where $\beta$ is the shear wave velocity, $\eta$ is the fraction of the rupture propagation velocity to $\beta$, and $z$ is a constant linked to maximum slip rate equal to 1.68 for an $\omega^{2}$ spectrum from the shear-dislocation theory.

Using the $\omega^{2}$ shape for each subfault, the Fourier acceleration spectrum from FINSIM for a single subfault is (Beresnev and Atkinson, 1998):

$$
A_{i j}(f)=\frac{C \Delta \sigma f^{2} \Delta l^{3}}{1+\left(\frac{\pi}{\eta z \beta}\right)^{2} f^{2} \Delta l^{2}}
$$

$N$ subfaults are summed to obtain the target seismic moment $\left(M_{0}\right)$. At frequencies above the corner frequency the spectra of radiation add incoherently, which leads to an amplitude increase by $N^{1 / 2}$ (Joyner and Boore, 1986). Following the process of Beresnev and Atkinson (1998) the final spectra is shown to have the form:

$$
A_{i j}(f)=C\left(\Delta \sigma M_{0}\right)^{1 / 2}\left(\frac{\eta z \beta}{\pi}\right)^{2} \Delta l^{-1 / 2}
$$

This shows a square-root dependence on $\Delta l$, implying that the final summed source spectra is dependent upon the choice of the number and size of the subfaults. This effect is shown in Figure 6 where the far-field received energy is calculated for different subfault sizes.

The input parameters needed for FINSIM are mostly the same as the parameters for SMSIM, but with some important differences. The geometry of the fault and subfault sizes must be defined in FINSIM, and the option for different types of sources is removed. Geographic coordinates are used for the fault and site locations instead of distance, which is used by SMSIM. In addition, a slip distribution and the position of the hypocenter, in terms of subfault columns and rows, must be defined. 


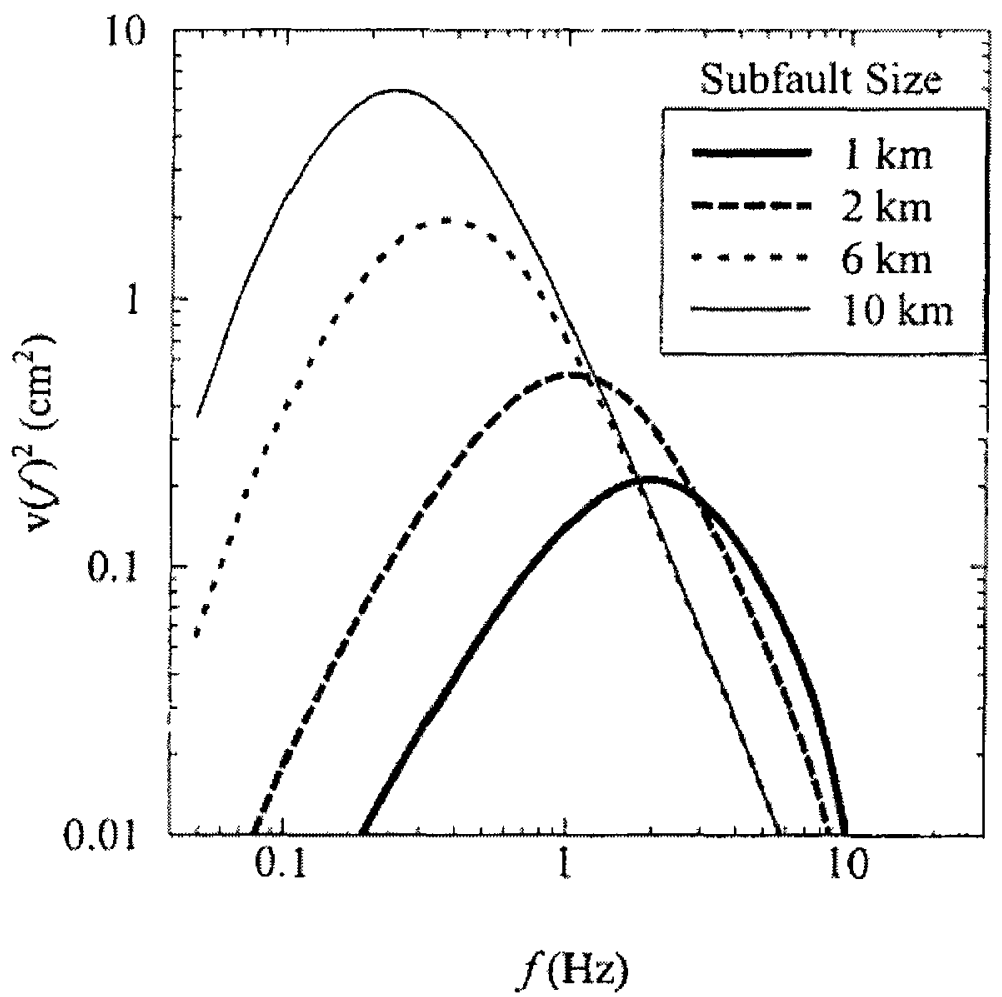

Figure 6: Far-field energy of a fault with different subfault lengths using FINSIM (Motazedian and Atkinson, 2005). The event is an $M 7.0$ earthquake at a distance of $333 \mathrm{~km}$, simulated using FINSIM (a static corner frequency approach). 


\subsubsection{EXSIM}

EXSIM (Motazedian and Atkinson, 2005) is an extended stochastic finite-fault modelling technique that uses the FINSIM program as a foundation to simulate earthquake acceleration time series. The motivation for EXSIM arises from the dependence of FINSIM on subfault size. The resulting ground motion from an earthquake should not depend on how the user decides to split the earthquake rupture plane into subfaults; the result should be the same, regardless of the number and size of the subfaults.

EXSIM implements a dynamic corner frequency (Motazedian and Atkinson 2005) which has the corner frequency of a subfault change as the number of previously ruptured subfaults changes. If $N_{R}(t)$ is the cumulative number of ruptured subfaults at time $t$, the corner frequency is:

$$
f_{0 l J}(t)=N_{R}(t)^{-1 / 3} 4.9 \times 10^{6} \beta\left(\frac{\Delta \sigma}{M_{0 a v e}}\right)^{1 / 3}
$$

where $f_{0 t J}(t)$ is the corner frequency of the $i$ th, $j$ th subfault at time $t$, and $M_{0 a v e}=M_{0} / N$ is the average seismic moment of the subfaults. Taking $t=t_{\text {end }}$ then $N_{R}\left(t_{\text {end }}\right)=N$ and the corner frequency becomes:

$$
f_{0 \iota J}\left(t_{\text {end }}\right)=N^{-1 / 3} 4.9 \times 10^{6} \beta\left(\frac{\Delta \sigma}{M_{0} / N}\right)^{1 / 3}=f_{0}
$$

This shows the corner frequency at $t_{\text {end }}$, is the corner frequency of the entire fault (Motazedian and Atkinson, 2005). This dynamic corner frequency reduces the level of the spectrum and the radiated energy of each subfault at high frequencies, reducing the subfault size dependency as shown in Figure 7. Comparing Figure 7 with Figure 6 shows one of the important differences between FINSIM and EXSIM.

In EXSIM, a scaling factor, $H_{l \jmath}$, is introduced to conserve the total radiated energy of 


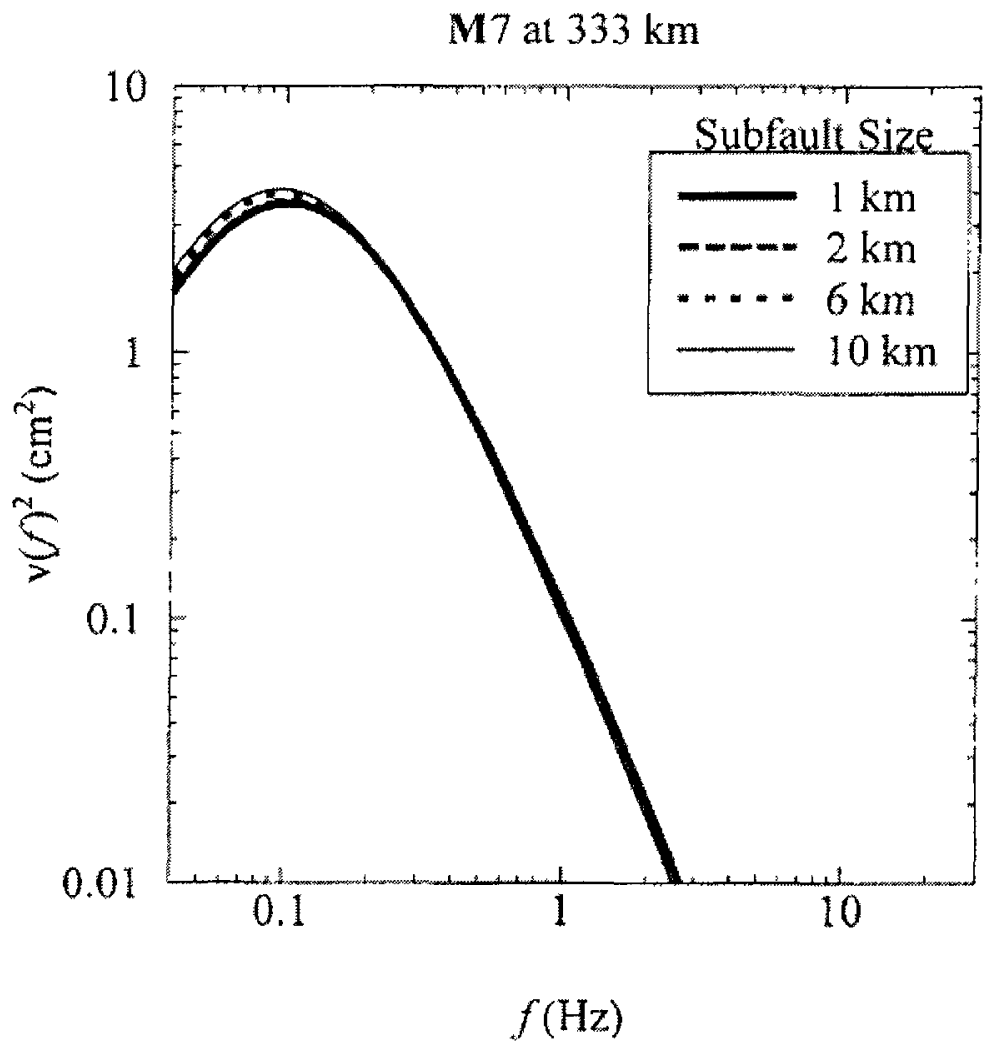

Figure 7: Far-field energy of a fault with different subfault lengths using EXSIM (Motazedian and Atkinson, 2005). The event is an $M 7.0$ earthquake at a distance of $333 \mathrm{~km}$, simulated using the dynamic corner frequency approach. 
high frequencies for the subfaults. The acceleration spectrum with this scaling factor is:

$$
A_{l j}(f)=\frac{C M_{0 l J} H_{l j}(2 \pi f)^{2}}{1+f / f_{0 \ell \jmath}}
$$

Requiring conservation of energy between the energy radiated from a subfault with the scaling factor and the average energy radiated from a subfault (Motazedian and Atkinson 2005), the scaling factor is:

$$
H_{l J}=\left(\frac{\sum\left(C M_{0}\left(\frac{2 \pi f}{1+\left(f / f_{0 \text { man }}\right)^{2}}\right) * h c\right)^{2} / N}{\sum\left(C M_{\text {0ave }}\left(\frac{2 \pi f}{1+\left(f / f_{0 \ell}\right)^{2}}\right) * h c\right)^{2}}\right)^{1 / 2}
$$

where $h c$ is the high frequency filter.

EXSIM also includes the option of having pulsing subfaults, which controls the slip duration at any location on the fault. In stochastic finite-fault modelling it is considered that only part of the rupture area actively contributes to the ground motion radiation, with the other areas being passive at those times. It is implemented by having the dynamic corner decrease in time up to a constant that is a fixed percent of the total rupture area.

\subsubsection{EXSIM_Beta}

EXSIM_Beta is the most recent version of EXSIM, with modifications from D.M. Boore, D. Motazedian, X.D. and Sun to make the simulated acceleration time series more accurate (Atkinson et al., 2009; Boore, 2009). The underlying physics is still the same as EXSIM. Some of the loops and the order of calculations have been changed to reduce unnecessary repetition of calculating constants throughout the process. Any memory limitations have been increased to a more satisfactory level, as memory allocations and processing times are at a more convenient level.

The changes introduce by Boore (2009) were motivated from a lack of consistent results 
when using EXSIM and SMSIM to simulate the same earthquake. Some of the inconsistencies were the result of the difference in methods between stochastic point-source and stochastic finite-fault modelling. To provide a better agreement between the two simulation techniques, there are some changes that were made to EXSIM, and a few were made to SMSIM. EXSIM_Beta was improved by basing the scaling of the high frequencies on the integral of the squared Fourier acceleration spectrum, not truncating the times series of each subfault, using the subfault corner frequency for the duration of motions from each subfault, and using a filter function to boost spectral amplitudes at frequencies less than but near the subfault corner frequency.

The main feature of EXSIM_Beta over the original EXSIM is the option of scaling the low frequencies using Motazedian's low-frequency coefficient. Since stochastic simulations are not well suited for reproducing the lower frequency portion of the Fourier spectrum, the option to either increase or decrease the low-frequency amplitude was added to EXSIM_Beta.

In this thesis research, another frequency scaling parameter, which is based on the fractional stress drop function (Brune, 1970), was added to EXSIM_Beta . Both techniques (using Motazedian's low-frequency coefficient and the fractional stress drop function) scale the lower frequency portion of the spectra without substantially affecting the high-frequency amplitudes.

This program is used in the same way as EXSIM. There is a controlling formatted input file for the parameters in a simulation. EXSIM_Beta produces four separate output files: the acceleration time series; a summary of the parameters; a summary of the specified values for sites; and, the Fourier acceleration spectra, along with the averages of pseudo-spectral acceleration, pseudo-spectral velocity and spectral displacement. 


\section{Chapter 3}

\section{Earthquake Parameters}

Different earthquake types and sizes release energy at different frequencies as the waves propagate and result in ground motions. These ground motions are recorded by seismometers at seismic stations. Using a large network of seismometers, it is possible to classify an earthquake in terms of the amount of energy released through magnitude scales, the movement type on the fault surface, the location of earthquake, and a large number of other parameters. These parameters can then be used to predict ground movement of future earthquakes.

The parameters used to classify earthquakes can be generalized into several different catagories, including source terms, crustal propagation, site effects, and instrumentation or type of motion recorded (Boore, 2003). Source terms determine the properties of the earthquake and range from the size of the fault plane to the type of motion, and the amount of energy released. Crustal propagation includes the effects of the ground motion attenuations, or changes in time duration, caused by the signal travelling through the earth.

The site effects explain what happens at a specific location and incorporate the effects of the local geological settings, and detail any surface or subsurface topography effects at a specific site. Instrumentation or type of ground motion recorded accounts for what equipment is used to measure the signal, along with what type of ground motion that was recorded. 
Although there are a large number of recordings for several earthquakes, the uncertainty associated with assigning a value to most of these variables is rather large. This is in part due to the random nature of earthquake ground motion, and the large variability of the high frequency content contained in a wave envelope. For most of the parameters a value assigned is taken as the mean value with a normal distribution and a standard deviation determined from the quality of the data set.

\subsection{Source Parameters}

Earthquakes are large and complicated processes that have varying amounts of energy released which can be closely determined from a long list of descriptive parameters. Parameters and variables that describe the earthquake and the earthquake process at the fault plane are called source parameters. These parameters determine the characteristics of the earthquake at the source, regardless of the path and site effects.

To completely describe the source term of an earthquake there are many factors and variables to take into consideration. The key is the determination of the number of parameters needed to give a complete or fairly accurate description of any earthquake. The most important parameters are the magnitude of the earthquake, which describes the size of the earthquake, and the energy the energy the earthquake releases.

In the thesis, moment magnitude is used for a couple reasons: it is the best description of energy released by an earthquake and has a direct correlation to seismic moment; and, it does not saturate at larger magnitudes as other types of magnitude scales.

Source parameters also include the fault geometry, fault mechanism and stress drop. All these parameters are important for the final calculation of response spectra produced by an earthquake and are described in Sections 3.1.1, 3.1.2 and 3.1.3, respectively. Other source parameters are the slip rate and pulsing percent, which are described in the EXSIM section of Chapet 2 (Section 2.2.2). These parameters contain information of the slip rate and the 


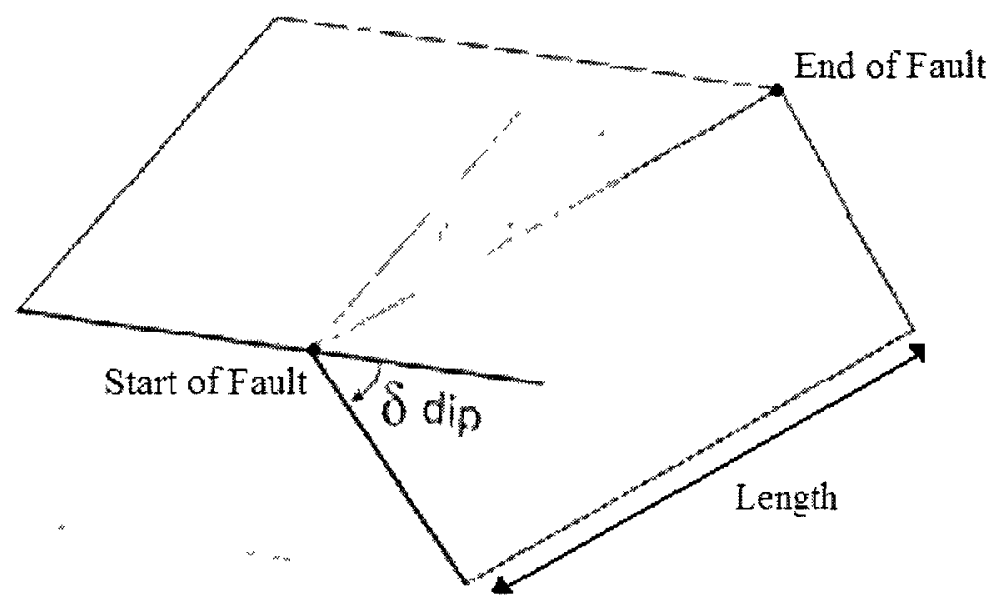

Figure 8: An illustration of the fault geometry.

slip duration on the fault plane (Motazedian and Atkinson, 2005). The slip weights matrix describes the relative contribution of each subfault to the overall time series (Beresnev and Atkinson, 1998a; Motazedian and Atkinson, 2005).

\subsubsection{Fault Geometry}

The fault plane is not a single parameter; rather, it is a collection of values to describe where an earthquake occurs. First, the latitude and longitude of the edge of the fault describe the location of where the fault starts. There are two ways of describing the rest of the fault: 1) strike, length, and dip angle; or, 2) latitude and longitude of the other edge of the fault which are shown in Figure 8. Using either of these approaches, the direction that the fault extends and the distance or length of the fault are known. The depth describes how deep the upper edge of the fault reaches, e.g. a depth of $0 \mathrm{~km}$ indicates the fault would have a surface rupture. The dip angle is the angle of the fault from the horizontal plane to the right of the strike angle. The width of the fault is how long the fault extends along the dip angle.

After the fault plane is set up for finite-fault modelling it is necessary to break up the fault into subfaults. The length and width of the subfaults follow the same description as 
the fault, except they are the size of the subfaults to be used. In EXSIM, the hypocentre location is given in terms of which subfault it occurs on, determined by two numbers, $i$ and $j$ which represent the appropriate subfault. This is the location where the earthquake originates and the rupture begins; it then propagates outwards in all directions that the fault extends.

It is worth adding a cautionary note on fault plane solutions and earthquake modelling: an earthquake rupture is not always the same as a fault plane. A fault describes an existing plane of breakage in the Earth, due to brittle fracture of the material. An earthquake rupture is the plane in which movement has occurred and the area of the earthquake. Not every earthquake on a fault will rupture over the entire fault plane, as it may only occur on a fraction of the entire fault. A good example is on the most studied fault in western North America - the San Andreas Fault. This fault extends over thousands of kilometres, yet the majority of the earthquakes on this fault may only rupture over a hundred kilometres or less. Please note that, in this thesis, any description of an earthquake fault plane is the area over which the earthquake has ruptured and not always the entire fault in that area.

\subsubsection{Earthquake Size}

The size of an earthquake can be defined in many different ways, but to have a simple number describing its effects is difficult. Aki (1967) described a parameter that is unique for each earthquake and is dependent upon several properties, including size. The seismic moment of an earthquake mathematically is:

$$
M_{0}=\mu A \bar{d}
$$

where $M_{0}$ (in $d y n e-\mathrm{cm}$ ) is the seismic moment, $\mu$ (in $d y n e / \mathrm{cm}^{2}$ ) is the rigidity taken as about $3 \times 10^{11}$ dyne $/ \mathrm{cm}^{2}, A$ (in $\mathrm{cm}^{2}$ ) is the area of the ruptured fault, and $\bar{d}$ (in $\mathrm{cm}$ ) is the average displacement along the fault. This number can be very large and generally spans 
several orders of magnitude.

Magnitude scales were created for the purpose of having a simple number to represent the power of an earthquake. There are many different types of magnitude scales, all of which are based on a logarithmic scale, in order to accommodate the large range of earthquake sizes. The magnitude scale introduced by Kanamori (1977) is a convenient scale based on the total energy of an earthquake. Moment magnitude, $M_{w}$ given by (Hanks and Kanamori, 1979):

$$
M_{w}=\log M_{0}-10.7
$$

where $M_{0}$ is in dyne-cm and is a magnitude scale that uses the seismic moment, but is represented by a number similar to what other magnitude scales give.

\subsubsection{Stress Drop}

Stress drop, $\Delta \sigma$, is defined as the amount of stress released during an earthquake or the difference in stress before and after an earthquake. In a mathematical sense, it is:

$$
\Delta \sigma=\sigma_{i}-\sigma_{f}
$$

where $\sigma_{i}$ is the initial stress and $\sigma_{f}$ is the stress after an earthquake has occurred.

The value of $\Delta \sigma$ is impossible to measure, yet it proves to be an essential role in determining high-frequency ground motions: it is also a common attribute in other earthquake parameters and characteristics. Because it is an essential part of predictive earthquake equations, it is important to have a useful value for stress drop that will provide accurate simulations of ground motions.

Great care is taken into assigning stress drop values, due to of its importance in controlling levels of ground motion spectra, specifically the high-frequency spectra. It is not unusual for the stress drop to need a larger value in stochastic simulations (Moghaddam et 
$a l ., 2010)$ to produce the required level of recorded ground motion spectra. This discrepancy is cause for concern, since stress drop values taken from other methods may produce lower values of ground motion parameters used for engineering purposes.

\subsubsection{Rupture Process}

An earthquake rupture is a quick and complicated process. It usually occurs over a matter of seconds, yet it releases a tremendous amount of energy in every direction. When the amount of stored stress energy surpasses the static friction threshold on a fault, the earthquake rupture begins. The starting point is called the hypocentre and is generally marked on a map using the epicentre, which is the point on the Earth's surface directly above the hypocentre. The rupture then propagates along the fault plane in all directions from the hypocentre at a speed which is represented by a proportional amount of the S-wave velocity. The fraction of the rupture velocity over the S-wave velocity is represented by $\eta$ as:

$$
\eta=\frac{\text { rupture velocity }}{S-\text { wave velocity }}
$$

The values used for this study have a S-wave velocity of $3.7 \mathrm{~km} / \mathrm{s}$ and $\eta$ is taken as 0.8 from the average values in western North America.

The slip distribution of an earthquake is not equal over the entire fault, so there are areas of the fault that are displaced more than other areas. The areas that have greater displacement contribute more to the earthquake ground motions. In finite-fault modelling this effect is represent by the slip weight matrix (Beresnev and Atkinson, 1998a; Motazedian and Atkinson, 2005). The matrix represents the relative contribution of a subfault to the overall earthquake. In stochastic finite-fault modelling techniques, the overall weight of a subfault is determined by:

$$
w_{l j}=\frac{s w_{l j}}{\sum_{l=1}^{n l} \sum_{J=1}^{n w} s w_{l j}}
$$

where $w_{l j}$ is the overall slip weight of the ith and $j t h$ subfault, and $s w_{l j}$ is the relative 
slip weight. The sum of all slip weights is taken into account when calculating the overall contribution, enabling any matrix to be used as the relative slip weight matrix. There is one constraint to the values in the slip weight matrix: they should not be negative values.

\subsubsection{Source Models}

The source function for simulating earthquakes, $E\left(M_{0}, f\right)$ which is introduced in Chapter 2 (Section 2.1), can be expressed as (Boore, 2003):

$$
E\left(M_{0}, f\right)=C M_{0} S\left(M_{0}, f\right)
$$

where $C$ is a constant, $M_{0}$ is the seismic moment, and $S\left(M_{0}, f\right)$ is the displacement source spectrum. The constant in the above equation is calculated as:

$$
C=\frac{R_{\theta \phi} V F}{4 \pi \rho \beta^{3} R_{0}}
$$

where $R_{\theta \phi}$ is the radiation pattern averaged over a suitable range of azimuth and take-off angles (Boore and Boatwright, 1984), $V$ is the partition of total shear wave energy, $F$ is the effect of free surface, $\rho$ is the density, $\beta$ is the shear wave velocity, and $R_{0}$ is the reference distance.

Table 1 is a summary of the values used in the simulations. A two-corner frequency approach was introduced to model the finite-fault effects on ground motions (Atkinson and Boore, 1995; Atkinson and Silva, 2000; Moghaddam et al., 2010). The two-corner frequency source model, $S\left(M_{0}, f\right)$ is given by the equation (Boore, 2003):

$$
S\left(M_{0}, f\right)=S_{a}\left(M_{0}, f\right) \times S_{b}\left(M_{0}, f\right)
$$

$S_{a}$ and $S_{b}$ differ, depending on the model used shown in Table 2. The moment dependence is given by the relations between the corner frequencies $f_{a}$ and $f_{b}$, which appear in the 


\begin{tabular}{cc}
\hline Parameter & Value \\
\hline$R_{\theta \phi}$ & 0.55 \\
$V$ & $1 / \sqrt{2}$ \\
$F$ & 2 \\
$\rho$ & $2.8 \mathrm{~g} / \mathrm{cm}^{3}$ \\
$\beta$ & $3.7 \mathrm{~km} / \mathrm{s}$ \\
$R_{0}$ & $1 \mathrm{~km}$ \\
\hline
\end{tabular}

Table 1: A summary of the values contained in the constant of the source function.

\begin{tabular}{ccc}
\hline Model & $S_{a}$ & $S_{b}$ \\
\hline Atkinson and Boore $(1995)^{*}$ & $\frac{1-\varepsilon}{1+\left(f / f_{a}\right)^{2}}+\frac{\varepsilon}{1+\left(f / f_{b}\right)^{2}}$ & 1 \\
Frankel et al. (1996) & $\frac{1}{1+\left(f / f_{a}\right)^{2}}$ & 1 \\
Haddon (1996) & $\frac{1}{\left(1+\left(f / f_{a}\right)^{8}\right)^{1 / 8}}$ & $\frac{1}{\left(1+\left(f / f_{b}\right)^{8}\right)^{1 / 8}}$ \\
\hline
\end{tabular}

Table 2: The reference source models for determining the overall source model contribution. ${ }^{*} \varepsilon$ is a scaling factor used for this model and is not the same value as fractional stress drop. ${ }^{\dagger}$ is the $\omega^{2}$ model

factors and the seismic moment shown in Table 3. These different sources are primarily used for different point-source modelling.

In stochastic finite-fault modelling, the source function is usually taken as the $\omega^{2}$ model $(\omega=2 \pi f)$. The $\omega^{2}$ source model ( $\omega$-squared model) comes from Aki (1967), where it was constructed by fitting an exponential function to the autocorrelation function of the dislocation velocity of the source. According to Aki (1967), the source amplitude spectral

\begin{tabular}{cccc}
\hline Model & $\log f_{a}$ & $\log f_{b}$ & $\log \varepsilon$ \\
\hline Atkinson and & $M \geq 4.0: 2.41-0.533 M$ & $1.43-0.188 M$ & $2.52-0.637 M$ \\
Boore (1995)* & $M<4.0: 2.678-0.5 M$ & $2.678-0.5 M$ & 0.0 \\
Frankel et al. (1996) & $2.623-0.5 M$ & - & - \\
Haddon (1996) & $2.3-0.5 M$ & $3.4-0.5 M$ & - \\
\hline
\end{tabular}

Table 3: Corner frequencies and moment ratios. ${ }^{\dagger}$ is the $\omega^{2}$ model 
density is:

$$
|A(\omega)|=\frac{w D_{0} L}{\left\{1+\left(\frac{\cos \theta}{c}-\frac{1}{v}\right)\left(\frac{\omega}{k_{L}}\right)^{2}\right\}^{1 / 2}\left\{\left(1+\frac{\omega}{k_{T}}\right)^{2}\right\}^{1 / 2}}
$$

where $D_{0}$ is the average dislocation, $\mathrm{L}$ is the fault length, $w$ is the fault width, $\theta$ is the polar angle for spherical coordinates, $c$ is the wave velocity, $v$ is the rupture velocity, $k_{T}$ is the characteristic frequency, $k_{L}$ is the characteristic length.

Using assumptions of similarity, the source parameters were reduced to only one surface-wave magnitude as the scaling parameter. These assumptions of similarity show the corner frequency spectral amplitude is dependent upon the inverse cube power of the corner frequency and is a constant stress drop model. This is a good basis for predicting ground motions and has been used and adapted for various models (Brune, 1970; Boore, 1983; Beresnev and Atkinson 1997; Beresnev and Atkinson, 1998a; Boore, 2003; Motazedian and Atkinson, 2005). This source model accurately describes the general behaviour of the seismic spectrum.

\subsection{Regional Path Effects}

After an earthquake occurs, all produced waves propagate through the Earth. As a wave travels through the Earth's crust, some of its properties are changed, including the signal duration and the amplitude of the motion. These effects are strongly dependent on the distance between the source and the location where it is recorded, but also on the material through which it is propagating. All of the effects have distance as a factor, and most of the equations have distance as the dominant parameter.

There are other factors contributing to seismic wave propagation, including reflections of seismic waves from layered geological units. The strength and depth of the Moho discontinuity in the region affects the amount of reflected and refracted waves. The quality and strength of the material through which the seismic waves propagate determines how 
much energy is lost through wave propagation.

One of the regional path effects is crustal amplification. This arises from several contributions with the most important being a change in depth and also density as the seismic wave propagates. Most shallow earthquakes occur at, or at least extend to, a depth of at least several kilometres below the surface of the earth. At those depths, the material is more dense and has a higher seismic wave velocity. Closer to the surface, the material becomes less dense, reducing the seismic wave velocities. This causes an amplification of the seismic wave amplitude, as energy must be conserved.

A generic crustal amplification factor is included along with the other effects discussed here. Even for a hard rock site, where the shear wave velocity is larger than $2 \mathrm{~km} / \mathrm{s}$, there is still amplification at larger frequencies.

\subsubsection{Path Duration}

The duration of an earthquake signal is changed by the distance between the source and the site. This is best determined from empirical data sets and is different around the world. Some areas follow a generic trilinear duration model:

$$
T= \begin{cases}T_{0} & \text { if } R<R_{0} \\ T_{0}+d_{1} *\left(R-R_{0}\right) & \text { if } R_{0}<R<R_{1} \\ T_{0}+d_{1} *\left(R_{1}-R_{0}\right)+d_{2} * R & \text { if } R_{1}<R<R_{2} \\ T_{0}+d_{1} *\left(R_{1}-R_{0}\right)+d_{2} *\left(R_{2}-R_{1}\right)+d_{3} * R & \text { if } R>R_{2}\end{cases}
$$

where $T_{0}$ is the source duration, $d_{1}, d_{2}$ and $d_{3}$ are the addition factors, $R$ is the distance, $R_{0}$ is the minimum distance (is usually considered $1 \mathrm{~km}$ for the sake of simplicity), and $R_{1}$ and $R_{2}$ are the corner distances. These parameters vary across different regions, depending on the layers and depths of different geologic units and the depth and strength of the Moho 
discontinuity.

The model used here is: $T_{0}=0.0 \mathrm{~s}, d_{1}=d_{2}=d_{3}=0.1, R=\sqrt{d^{2}+h^{2}}$ where $d$ is the epicentral distance and $h$ is the depth for each subfault, $R_{1}=100 \mathrm{~km}$, and $R_{2}=500 \mathrm{~km}$. These values are consistent with the western North American parameters (Beresnev and Atkinson, 2002), which has been taken as a simplified distance-dependent duration from Raoff et al. (1999).

\subsubsection{Geometric Attenuation}

Geometrical spreading reduces the amplitude of a seismic wave since the energy is dispersed over a larger area as the seismic waves propagate through the crust. The energy per unit area is proportional to the inverse of the radius squared $\left(E \propto R^{-2}\right)$, and the amplitude is the square root of the energy $(A=\sqrt{E})$. In theory, the seismic wave amplitude should be inversely proportional to the distance. This can often be seen; however, at larger distances, reflections and refractions between the surface and the Moho discontinuity trap and amplify the waves, reducing the inverse dependence.

The geometrical spreading for a point-source model or a subfault can be considered a piecewise continuous series as a function of distance (Boore, 2003):

$$
Z(R)= \begin{cases}\frac{R_{0}}{R} & R \leq R_{1} \\ Z\left(R_{1}\right)\left(\frac{R_{1}}{R}\right)^{p_{1}} & R_{1}<R \leq R_{2} \\ \vdots & \\ Z\left(R_{n}\right)\left(\frac{R_{n}}{R}\right)^{p_{n}} & R_{n}<R\end{cases}
$$

In EXSIM this has been reduced to a trilinear function with input values $r g 1$ and $r g 2$ for the distances; and, pow1, pow2, and pow3 as the different exponents of attenuation. For western North America, the values are taken as $40 \mathrm{~km}$, and $130 \mathrm{~km}$ as the distances, and 
$-1,-0.5$, and -0.5 as the attenuation exponents, respectively (Raoof et al., 1999). This creates a simpler bilinear form of the geometric attenuation in the area. The values for $r g 2$ and pow 3 are included as dummy values, which are needed in order for the program to run properly.

\subsubsection{Anelastic Attenuation and Quality Factor}

The anelastic attenuation is the loss of energy due to intrinsic mechanisms. The energy is mostly transferred into heat or friction of the individual particles as a wave propagates through a medium. This is represented by:

$$
\exp \left[-\frac{\pi f R}{Q(f) v}\right]
$$

where $R$ is the distance, $f$ is the frequency, $v$ is the velocity of the seismic wave, and $Q(f)$ is the frequency dependent quality factor.

The quality factor is defined as:

$$
Q=-\frac{2 \pi \times \text { elastic energy stored in the wave }}{\text { energy lost in one cycle or wavelength }}
$$

This factor is also dependent upon the frequency of the propagating wave. It is a very difficult property to measure and many studies are being conducted upon it. For this study, the quality factor is taken as:

$$
Q(f)= \begin{cases}Q_{0} f^{\eta} & Q \geq Q_{m i n} \\ Q_{m i n} & Q<Q_{m i n}\end{cases}
$$

where $Q_{0}=180, \eta=0.45$, and $Q_{m i n}=1000$ (Raoof et al., 1999). These values agree best with the different studies in the area of western North America (Atkinson and Silva, 1997; 
Benz et al., 1997; Harmsen, 1997).

\subsection{Site Effects}

Seismic amplitude is also affected by amplification and deamplification factors. These effects are categorized into site effects, which are different at each location. There are several factors that contribute to these effects, including impedance contrast, resonance, spatial variability, high-frequency attenuation, and nonlinear effects. To account for everything, extensive studies at any particular location should be done. However, there are some generalizations that can be made to simplify this process and produce an accurate site response.

The Earth is known to be an efficient high-frequency filter, since the high frequency spectrum falls off quickly. There are several ways to model this behaviour with two common methods: the $f_{\text {max }}$ filter (Hanks, 1982; Boore, 1983), and the $\kappa_{0}$ filter (Anderson and Hough, 1984). For the purpose of this study, the latter was chosen, since the exponential decay of the acceleration spectrum at high frequencies is a good fit to the recordings. The $\kappa_{0}$ filter is:

$$
\exp \left(-\pi \kappa_{0} f\right)
$$

with $\kappa_{0}=0.035 \mathrm{~s}$ as our value, which is an average value taken for western North America (Boore and Joyner, 1997).

There are also frequency dependent amplification factors at the site, as a result of the material through which the waves travel. It is widely known that softer materials, such as soil and soft rocks, amplify earthquake ground motion. These amplifications can be classified in many different ways; however, a convenient classification method is the National Earthquake Hazard Reduction Program (NEHRP) class building codes. As the class code changes, mostly from a change in the $V_{s_{30}}$ (shear-wave velocity at a depth of 30 metres), the amplifications at different frequencies vary. 


\begin{tabular}{|c|c|}
\hline Frequency $(\mathrm{Hz})$ & Amplification Factor \\
\hline 0.5 & 1.00 \\
\hline 1.0 & 1.13 \\
\hline 2.0 & 1.22 \\
\hline 5.0 & 1.36 \\
\hline 10.0 & 1.41 \\
\hline
\end{tabular}

Table 4: Generic crustal amplification from Atkinson and Boore (2006) with assumed shear wave velocity of $\geq 2 \mathrm{~km} / \mathrm{s}$

Table 4 is the site amplification used for hard rock sites (i.e. NEHRP A), but is taken as a generic frequency dependent crustal profile for our application. This amplification is empirical and based upon Siddiqqi and Atkinson (2002). Boore and Joyner (1997) found generic amplifications at node frequencies for a few different site classes, based upon $V_{s_{30}}$ values. The two used here are the amplifications for the NEHRP C and NEHRP D class sites shown in Tables 5 and 6, respectively.

There are a few rare sites where the site response is much different from these modelled effects. This can be due to different factors, including subsurface topography, which generally has not been modelled, and must be looked at an individual basis. There are several different methods determine a site response using the seismic recordings or other measurements; however, they are beyond the scope of this research. Therefore, these sites have been classified as inaccurate and have been disregarded for the purpose of this study.

\subsection{Earthquake Spectrum}

Earthquake source spectra are dominated by higher frequency signals; and, often the lower frequencies are overlooked, since the signals can be orders of magnitudes lower. However the low frequency spectrum is critical for engineering purposes, as they are often more destructive to large infrastructure. The stochastic methods are based on high-frequency 


\begin{tabular}{|c|c|}
\hline Frequency $(\mathrm{Hz})$ & Amplification Factor \\
\hline 0.01 & 1.00 \\
\hline 0.09 & 1.10 \\
\hline 0.16 & 1.18 \\
\hline 0.51 & 1.42 \\
\hline 0.84 & 1.58 \\
\hline 1.25 & 1.74 \\
\hline 2.26 & 2.06 \\
\hline 3.17 & 2.25 \\
\hline 6.05 & 2.58 \\
\hline 16.6 & 3.13 \\
\hline 61.2 & 4.00 \\
\hline
\end{tabular}

Table 5: Frequency dependent amplification factors for NEHRP class C sites (Boore and Joyner, 1997)

\begin{tabular}{|c|c|}
\hline Frequency $(\mathrm{Hz})$ & Amplification Factor \\
\hline 0.01 & 1.00 \\
\hline 0.09 & 1.43 \\
\hline 0.16 & 1.71 \\
\hline 0.51 & 2.51 \\
\hline 0.84 & 2.92 \\
\hline 1.25 & 3.10 \\
\hline 2.26 & 3.23 \\
\hline 3.17 & 3.18 \\
\hline 6.05 & 3.18 \\
\hline 16.6 & 3.18 \\
\hline 61.2 & 3.18 \\
\hline
\end{tabular}

Table 6: Frequency dependent amplification factors for NEHRP class D sites (Boore and Joyner, 1997). 
random noise, and the low-frequency content is dominated by the shape of the envelope; therefore, stochastic methods are not ideal for predicting lower frequencies.

The shape of the envelope in stochastic modelling is, however, determined by the window used in step 2 in Figure 3, making the low-frequency signal in stochastic models more deterministic than the rest of the spectrum. There are several ways to accommodate the low-frequency spectrum in the EXSIM program. These methods use a different process to determine the level of low-frequency ground motion.

\subsubsection{Boore's Low Frequency Taper}

Boore's taper attempts to correct for the incoherent summation of the subfault spectra for frequencies less than the subfault corner frequency (Frankel, 1995b). It uses the number of subfaults and the scaling factor, $H_{l \jmath}$, to create a scaling level:

$$
\text { taper }=\frac{\sqrt{\# \text { of Subfaults }}}{H_{\imath J}}
$$

The taper frequency is determined as:

$$
\text { taper frequency }=\frac{f_{0_{\imath j}}}{\sqrt{\text { taper }}}
$$

This taper is then used to modify the acceleration spectra of a subfault by modifying the scaling factor. The new frequency dependent scaling factor becomes:

$$
H_{I_{\text {Boore }}}=H_{i J} \times \operatorname{taper} \times \frac{1+\left(\frac{f}{f_{0_{l j}}}\right)^{2}}{1+\left(\frac{f}{\text { taper frequency }}\right)^{2}}
$$

Figure 9 shows the effect of adding this taper to a simulated psuedo spectral acceleration (PSA) spectrum. The simulation with Boore's taper has a higher amplitude at frequencies below about $1 \mathrm{HZ}$ and the same amplitudes above. This taper uses parameters already 


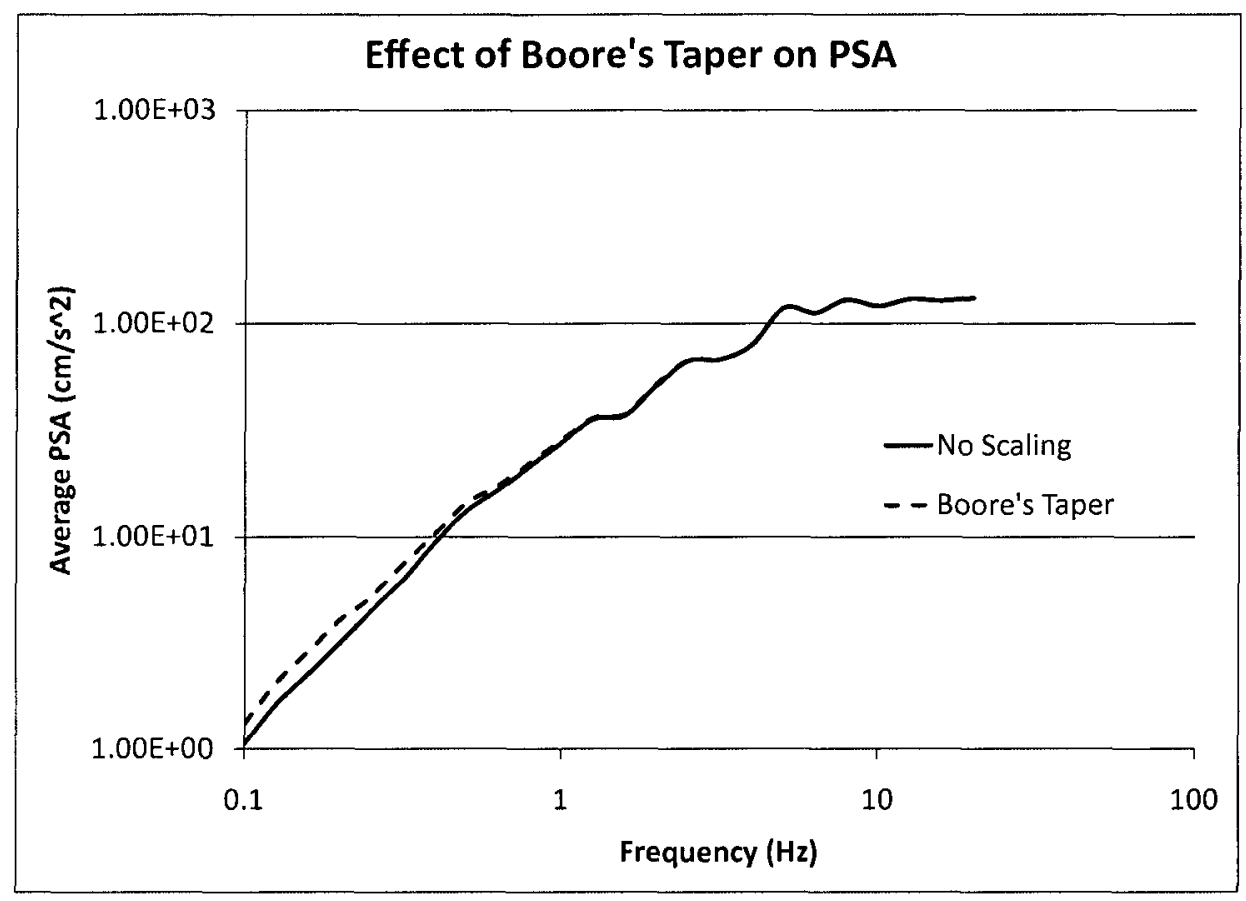

Figure 9: The effect on PSA values of adding Boore's taper to an earthquake simulation. The earthquake magnitude is $M 6.6$ at a distance of $55 \mathrm{~km}$ and a stress drop of $33 \mathrm{bars}$. Other parameters follow the generic earthquake parameters found in Table 23.

known within the stochastic simulation; however, this provides no control over the level of low-frequency scaling. This is important for comparison with recordings that have higher or lower spectra at the lower frequencies. Boore's taper does not require any additional parameters and uses the corner frequency and the number of subfaults to taper the lowfrequency spectrum.

\subsubsection{Motazedian's Low-Frequency Coefficient}

Motazedian's low-frequency coefficient was introduced as a way to control the level of low-frequency spectra in a stochastic simulation. In EXSIM, it is a simple addition that 
which scales the corner frequency of the hypocentre subfault.

$$
f_{0_{\text {hypo }} L F C}=(1-\text { Motazedian's LFC }) f_{0_{\text {hypo }}}
$$

This coefficient creates a ratio between the high-frequency and low-frequency spectra, which can be modified as needed. Figure 10 shows the effect on the PSA spectra when the low frequency coefficient is adjusted. In this case, the higher frequency portion of the spectra is changed and should be adjusted after applying this low-frequency scaling method. This is useful for matching the simulated low frequency spectra to recorded levels, as there is currently no process for estimating this value for future events. Motazedian's low-frequency coefficient applies a multiplication factor to the subfault corner frequencies.

\subsubsection{Fractional Stress Drop}

In most cases, the stress drop of an earthquake appears to be a small fraction of the effective stress (Aki, 1967; King and Knopoff, 1968; Brune, 1970): a similar effect occurs in laboratory experiments called 'stick slip' (Brace and Byerlee, 1966). There are several different mechanisms that can cause the average stress drop to be less than $100 \%$ of the effective stress, including the rupture plane locking itself after the rupture has passed. In this case, the rupture can travel a large distance with the total effective stress acting for only a short time when the rupture passes a certain point (Brune, 1970). This effect can be modelled by applying a reverse stress of $1-\varepsilon$, which multiplies the Fourier acceleration spectrum by the function (Brune, 1970; Brune, 1971):

$$
F(\varepsilon)=\left\{[2-2 \varepsilon]\left[1-\cos \left(\frac{1.21 \varepsilon \omega}{\alpha}\right)\right]+\varepsilon^{2}\right\}^{\frac{1}{2}}
$$

where $\alpha=2.34 \beta / r$ (Brune, 1971).

At low frequencies, the function $F(\varepsilon)$ approaches $\varepsilon$, multiplying the low-frequency 


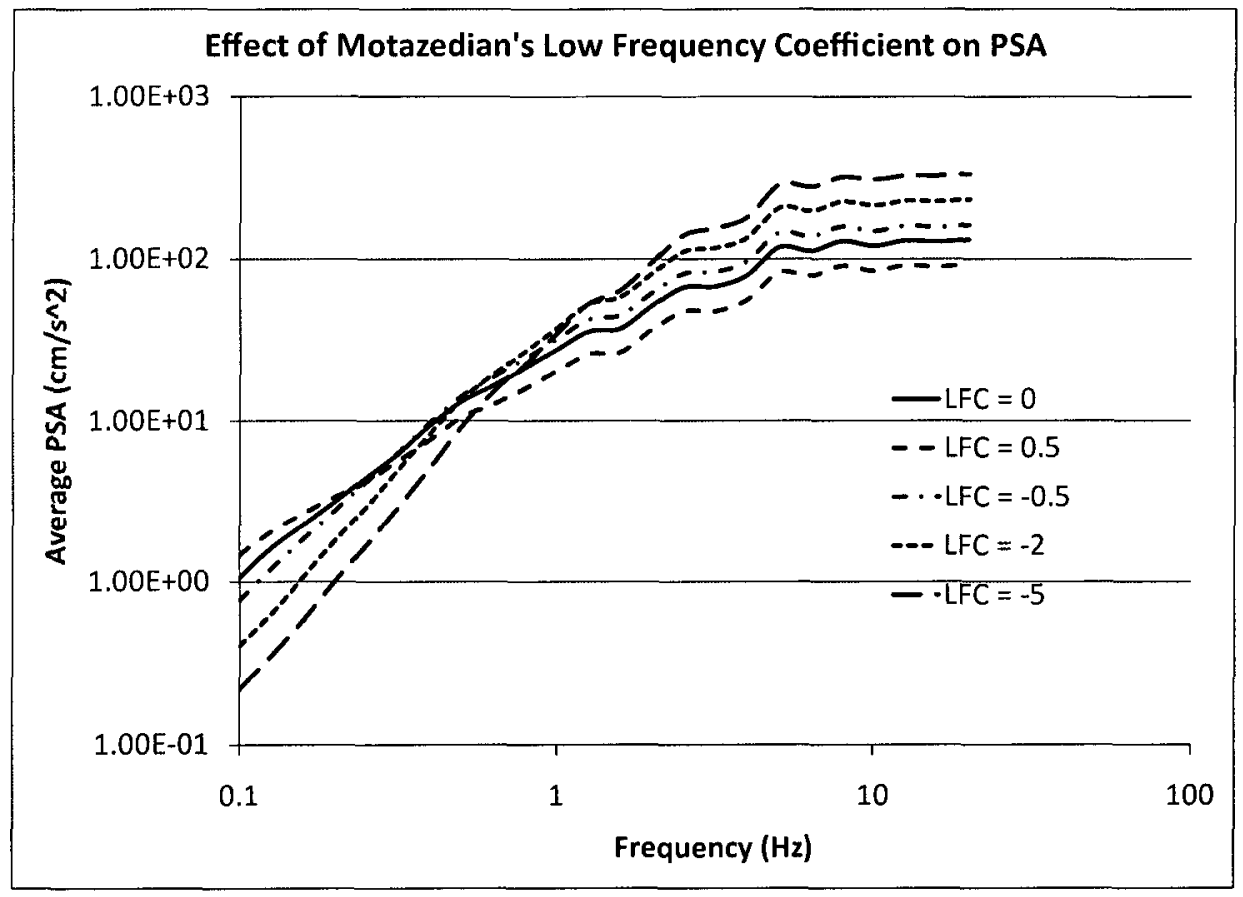

Figure 10: The effect on PSA values when Motazedian's low-frequency coefficient $(L F C)$ is adjusted. The earthquake magnitude is $M 6.6$ at a distance of $55 \mathrm{~km}$ and a stress drop of 33 bars. Other parameters follow the generic earthquake parameters found in Table 23. 
spectra by the fractional stress drop. Figure 11 displays the effect on PSA when $\varepsilon$ is adjusted. If $\varepsilon$ is above 2 or below 0.01 , the entire spectrum is shifted. Between these two values, only the lower frequency portion changes value with the higher frequencies matching closely. The higher frequency amplitudes are not exactly the same, due to the oscillation of $F(\varepsilon)$ at larger $\omega$ values.

This function, when applied to the stochastic simulations, also provides a ratio between the high-frequency and low-frequency spectra as a basis to what the value of fractional stress drop should be. The value of $\varepsilon$ depends on the tectonic conditions surrounding the fault plane and the amount of stress released through earthquakes. Fractional stress drop applies a function to the spectrum based on the amount of stress used in the rupture compared to the amount of stress available. 


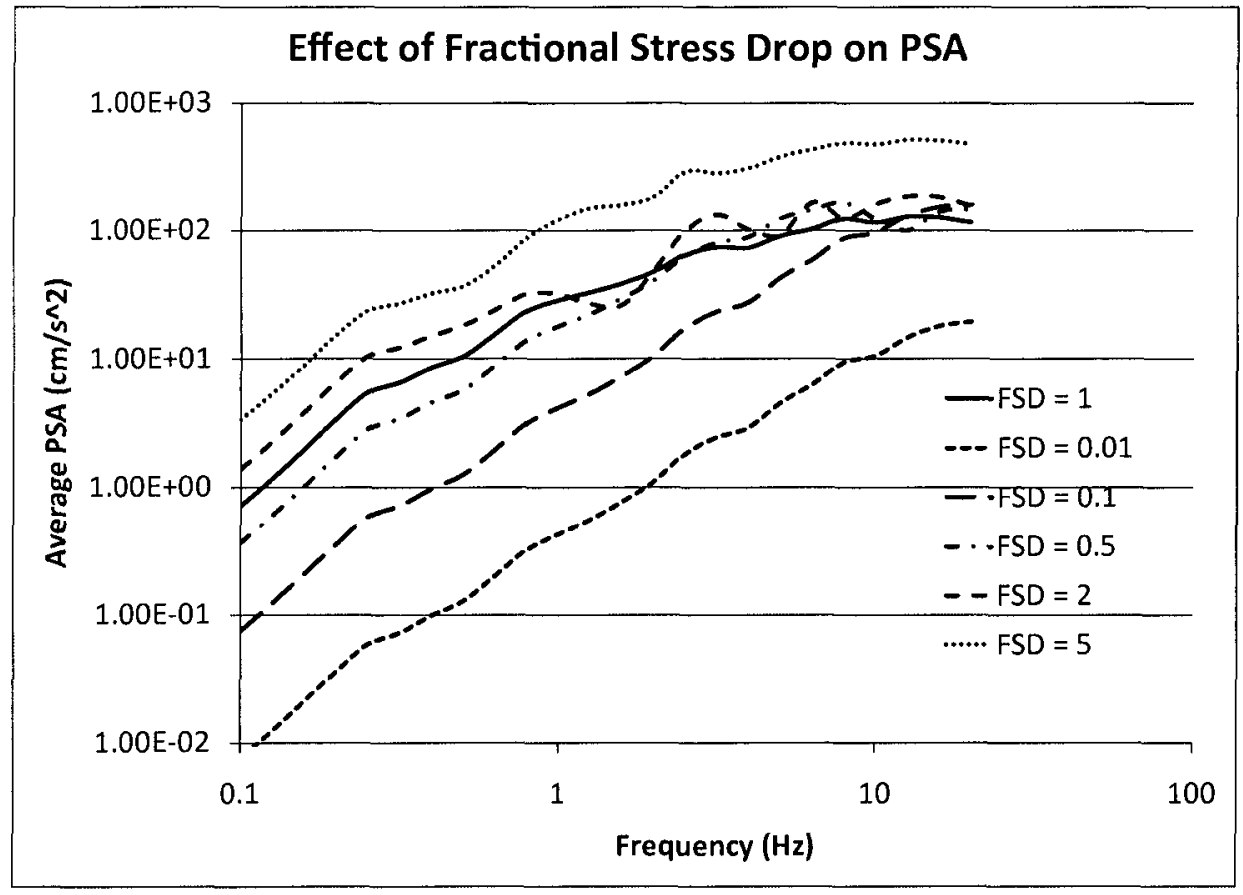

Figure 11: The effect on PSA values when fractional stress drop $(F S D)$ is adjusted. The earthquake magnitude is $M 6.6$ at a distance of $55 \mathrm{~km}$ and a stress drop of $33 \mathrm{bars}$. Other parameters follow the generic earthquake parameters found in Table 23. 


\section{Chapter 4}

\section{EXSIM Modifications}

Increasing the accuracy of EXSIM without making the input overly complex would be ideal, as with any earthquake modelling technique. Although there will always be an error associated with the values given in earthquake modelling and the spectra from earthquake simulations, it is possible to reduce the error to a better level of acceptability. After studying the EXSIM program in detail and research into known earthquake behaviour, several changes have been applied to the EXSIM program. These changes will hopefully improve the overall performance of EXSIM and still be relatively simple enough to be a useful resource for a wide range of purposes related to earthquake engineering and engineering seismology.

The changes proposed include "cleaning" up of the source code, and the application of new techniques in calculating several of the needed values. Specifically, the changes are:

1. The way EXSIM iterates over several hypocentres;

2. Calculation the number of effective subfaults in a different manner, mainly at the earlier rupture times;

3. The option of segmenting the fault plane; and,

4. Addition of a fractional stress drop term (Brune, 1970).

Although some of the changes require more or different input parameters, the overall complexity of the input has only changed a little. 


\subsection{Several Hypocentres}

A couple of changes made to EXSIM_Beta apply to the hypocentre. The first was to remove calling locateHypocentreRandomly() (see EXSIM code) from within the number of sites loop. This prevents different hypocentres being used for different sites, if there was more than one hypocentre being called, within the duration of the program.

The second adjustment was the addition of a loop for a different number of hypocentres. There is now an option to run the simulations over several hypocentres, up to a maximum 10. Each of the hypocentre iterations uses the same number of trials and sites to compute the average acceleration time series for the different sites according to the position of the hypocentre. If there is more than one hypocentre being iterated, the first hypocentre can be defined, but the other hypocentres are located randomly to ensure a variation of the hypocentre location.

\subsection{Number of Effective Subfaults}

The main appeal of EXSIM over the earlier FINSIM is the dynamic calculation of the corner frequency for subfaults. The corner frequency of a subfault contains the number of previously ruptured subfaults, which is dependent on the NoOfEffectiveSubfaults variable in the program. Formerly, this variable was the number of subfaults multiplied by the pulsing percent and divided in half, due to double-sided propagation, for the sake of simplicity. Now the variable contains a distance dependent relationship where it does not get divided in half if the distance from the centre of the subfault to the hypocentre is less than the radial distance of the length and width of the fault multiplied by the pulsing percent. The purpose is the confinement of an earthquake into one radial propagating part, until it becomes fragmented into two separate parts propagating in different directions. 


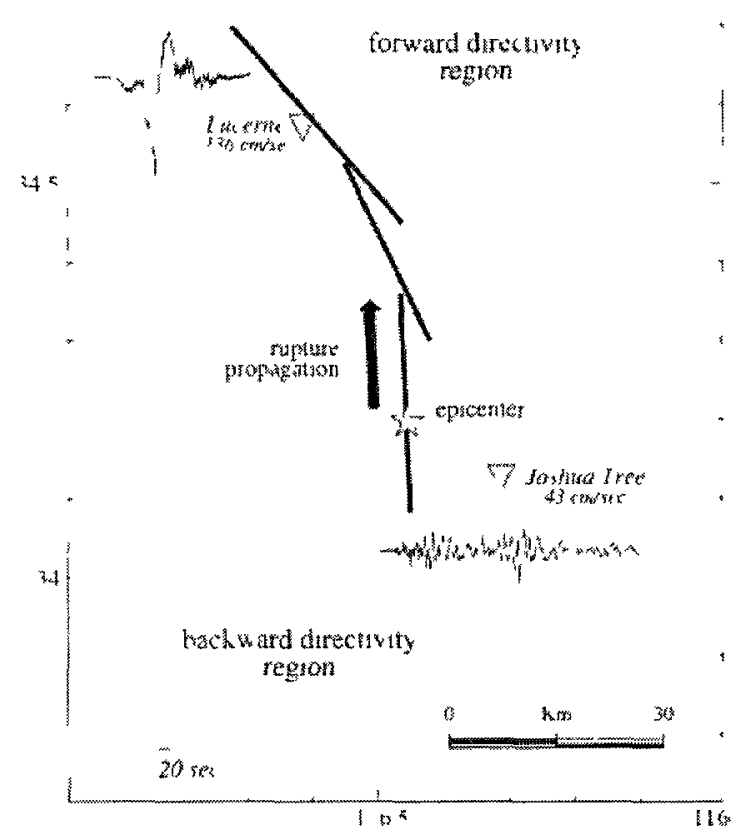

Figure 12: Map of the Landers region showing the location of the 1992 Landers earthquake. This earthquake occurred on three fault segments (Sommerville et al., 1997).

\subsection{Fault Segments}

The EXSIM_Beta program assumes that there is one rectangular fault for earthquakes of all sizes. This assumption works well when the earthquake fault can be reasonably modelled as a heterogeneous slip on one rectangular fault. However, in some cases, such as the Landers M7.4 earthquake, the earthquake fault does not fit on just one rectangular fault, because the earthquake fault is segmented and its segments have different orientations or azimuths as shown in Figure 12. In these cases, a single rectangular fault may give false distances for near-source sites, introducing an inaccuracy in the estimation of earthquake ground motions.

In the updated EXSIM program, the user can input an earthquake as being composed of 
one or more rectangular fault segments, up to a maximum of ten. This effectively breaks up the source of larger earthquakes when a fault may have ruptured along several planes from one location to another. The subfault distances to the site along with the time delays for individual subfault time series, are calculated more effectively and result in a more accurate final time series.

Assigning multiple fault segments to an earthquake source in the EXSIM program is different from conventional definitions of an earthquake fault. First, the user must decide how many fault segments there are, along with where to start and end each one. The input file requires the fault segments to be in order, starting from one end of a fault, to the next closest subfault, until the last fault segment, which contains the other end of the fault. The starting point of the first fault segment and the ending point of the last fault segment should be the open ends of the entire fault. The starting and end locations of the other fault segments should either be the same locations as the end of the previous fault segment or the starting point of the next fault segment, or be the position of the closest point to the previous or next fault segment.

Each fault segment must also be given a dip angle, which can be assigned as a negative value. A positive dip angle means the dip angle is to the right of the starting point in the direction along the fault and the strike angle is calculated from the start to the end point of a fault segment. A negative dip angle means the dip is to the left, causing the strike angle to be calculated from the end to the start point of the fault segment. Defining the fault segments accordingly allows the subfaults to be numbered correctly in terms of increasing from the beginning of the fault to the end.

Several values are calculated differently with the addition of multiple fault segments, including the time delay for a subfault and the subfault distance to the site. The time delay for a subfault remains unchanged if the subfault and the hypocentre are on the same fault segment. If the subfault is on a previous fault segment than the hypocentre, the time delay is determined from the summation of: 
1. The shortest distance from the hypocentre to the start of the fault segment on which it is located, divided by the rupture velocity;

2. The distance from the end of the fault segment on which the subfault is located on divided by the rupture velocity;

3. The distance between the end and start of any fault segments in between the subfault and hypocentre, divided by the shear wave velocity; and,

4. The length of any fault segments in between the subfault and hypocentre divided by the rupture velocity.

If the subfault is located on a fault segment following the hypocentre fault segment, the time delay is calculated similarly:

1. The distance from the hypocentre to the end of the fault segment, divided by the rupture velocity;

2. The distance from the subfault to the start of the fault segment on which it is located, divided by the rupture velocity;

3. Same as no 3. above: the distance between the end and start of any fault segments in between the subfault and hypocentre, divided by the shear wave velocity; and,

4. Same as no 4. above: the length of any fault segments in between the subfault and hypocentre divided by the rupture velocity.

The subfault distance is calculated using the same subroutine contained in EXSIM_Beta, but the values used are those obtained for the fault segment on which the subfault is located. The column value ( $i$ in the source code) is taken as either the column value of the subfault minus the column value of the start of the fault segment, or, if the dip angle is defined as a negative value, the column value of the end of the fault segment minus the column value of the subfault.

This feature of splitting large earthquake faults into different segments, allowing for better control of the fault geometry, includes better defined subfault distances over the entire fault. Figure 13 shows two sets of residuals, one using a segmented fault and the 


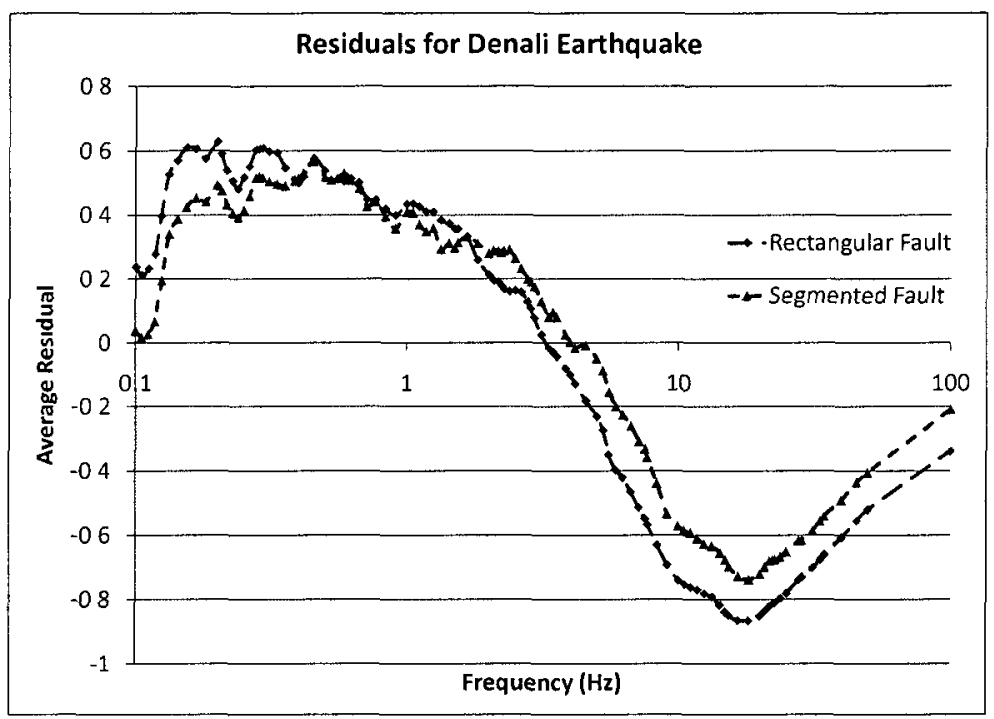

Figure 13: Average residual of the 2002 Denali M7.9 earthquake using EXSIM and EXSIM_Beta modelling techniques.

other using a rectangular fault for the Denali earthquake of 2002. The two faults as defined by the program are shown in Figure 14, along with the actual fault from the United States Geological Survey (USGS) earthquake summary. It is easier to get better agreement in the geometry using a fault with multiple segments for larger earthquakes, when the faults span upwards of hundreds of kilometres in length.

\subsection{Fractional Stress Drop}

The fractional stress drop, $\varepsilon$, is the fraction of the effective stress, or the total available stress, used during an earthquake. This effect is modelled by applying a reverse stress of $1-\varepsilon$ at a short time, $t_{d}$, after the initial shear stress (Brune, 1970). This multiplies the 


\section{Denali Earthquake of 2002}

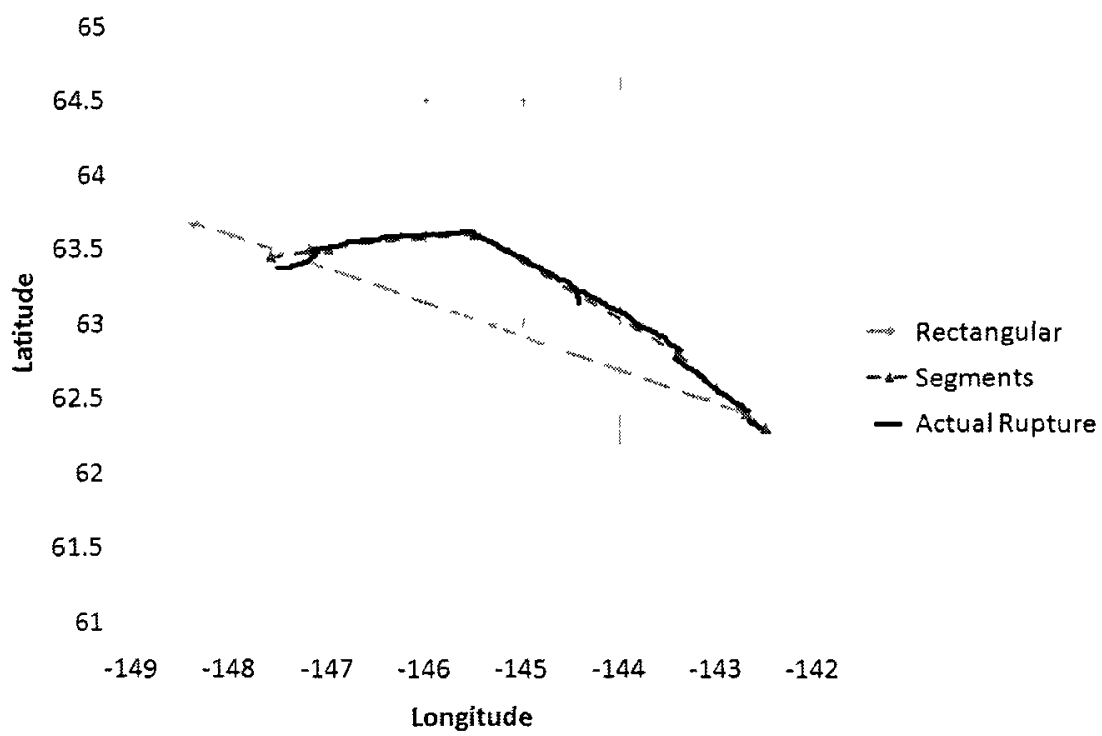

Figure 14: A map showing the faults used for the EXSIM simulations along with the actual ruptured fault. The actual fault is projected from the USGS report on the Denali Park, Alaska earthquake of November 3, 2002.

Fourier acceleration spectrum by:

$$
F(\varepsilon)=\left|1-(1-\varepsilon) e^{-t \omega t_{d}}\right|
$$

If $t_{d}$ is approximated as $\varepsilon$ times half of the total stress drop divided by the initial particle velocity, the resulting value for $t_{d}$ is $1.21 \varepsilon / \alpha$. Substituting this into the above function, the multiplication function for the Fourier acceleration spectrum becomes:

$$
F(\varepsilon)=\left\{[2-2 \varepsilon]\left[1-\cos \left(\frac{1.21 \varepsilon \omega}{\alpha(i)}\right)\right]+\varepsilon^{2}\right\}^{\frac{1}{2}}
$$

This function has little effect on higher frequencies as the function oscillates between $\varepsilon$ and $2-\varepsilon$ for larger values of $\omega$. When $\omega$ is small, the function approaches the value of $\varepsilon$. This function essentially scales the lower frequency spectra without significantly adjusting 


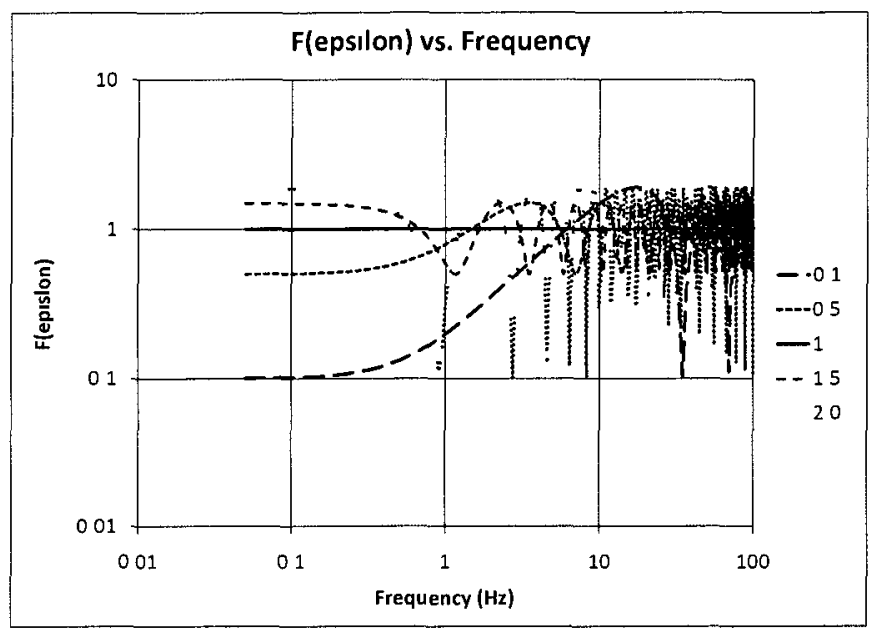

Figure 15: A plot of $F(\varepsilon)$ versus frequency for different values of $\varepsilon$.

the higher frequency spectra. In EXSIM, there is an added option for using this feature. Figure 15 shows the behaviour of $F(\varepsilon)$ for different $\varepsilon$ values.

Another parameter needed for fractional stress drop, but was not previously included in EXSIM, is $\alpha$, which is equal to $2.34 \frac{\beta}{r}$ (Brune, 1970; Brune 1971), where $r$ is the radius of an equivalent circular dislocation surface. In EXSIM, $\alpha$ is calculated after the input file is read and takes the value of:

$$
\alpha=2.34 * \frac{\beta}{\text { subFaultRadius }}
$$

where subFaultRadius $=\sqrt{(d l * d w) / \pi}, d l$ is the subfault length and $d w$ is the subfault width.

In the EXSIM program, where multiple fault segments are possible, the subfault width takes on an array value, and so too do the subfault radius and $\alpha$ value, depending on which 
fault segment the subfault is located. This changes the calculations slightly, with $\alpha$ calculated the same number of times as there are fault segments:

$$
\begin{gathered}
\alpha(i)=2.34 * \frac{\beta}{\text { subFaultRadius }(i)} \\
\text { subFaultRadius }(i)=\sqrt{(d l(i) * d w) / \pi}
\end{gathered}
$$

where $i$ is the fault segment on which the subfault is located.

Both the $\varepsilon$ and $\alpha$ values are passed into the function that calculates the source acceleration spectrum for the subfault at a given frequency. Another factor, called fepislon, is added into the final theoretical source spectra. If either Boore's taper or Motazedian's lowfrequency coefficient option is selected, this value is assigned as 1 and does not affect the original calculation in the previous EXSIM program. If fractional stress drop is selected as the low-frequency taper, the value is calculated by:

$$
\text { fepislon }=\left\{[2-2 \varepsilon]\left[1-\cos \left(\frac{1.21 \varepsilon \omega}{\alpha(i)}\right)\right]+\varepsilon^{2}\right\}^{\frac{1}{2}}
$$

which follows the original equation, i.e. equation (39).

The value of fepislon is also dependent on the frequency at which it is being calculated, since $\omega=2 \pi * f$. This function takes on a value between $\varepsilon$ and $|2-\varepsilon|$ as the cosine term fluctuates between 1 and -1 for the different values of $\omega$. For higher frequencies, this function oscillates between these two values with a mean of about $1.6-0.6 \varepsilon$; and, at lower frequencies, the value is roughly equal to $\varepsilon$ (Brune, 1970). It is another addition that encourages the low-frequency spectra to match closely with recorded values. It has some meaning behind it, in order to allow for a reasonable value of the fractional stress drop when using the stochastic method for predicting ground motions of future earthquakes.

Figures 16, 17 and 18 show the acceleration time series of the simulated shear wave for $M 5$ and $M 7$ earthquakes with several different values for $\varepsilon$. The different values of 

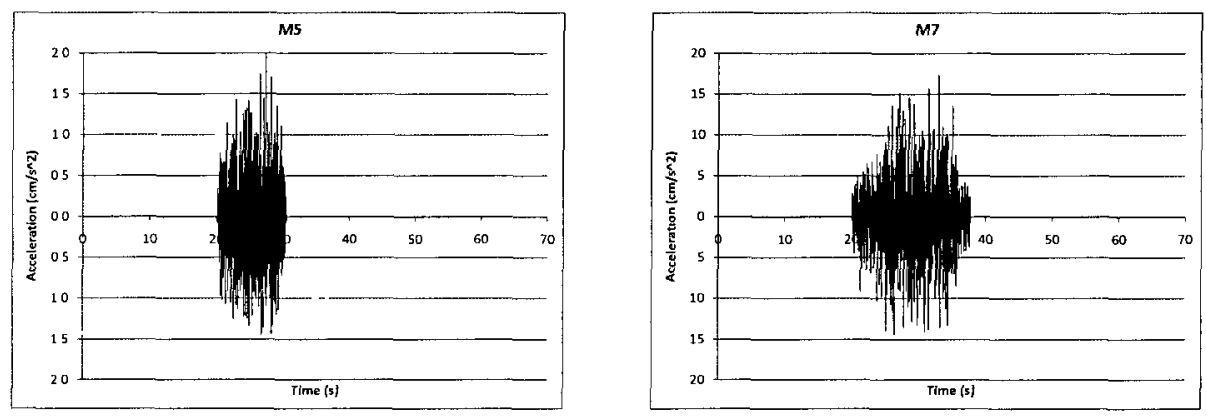

Figure 16: Simulated acceleration time series of $M 5$ and $M 7$ earthquakes at a distance of $100 \mathrm{~km}$ with $\varepsilon=1$ using a tapered boxcar window.
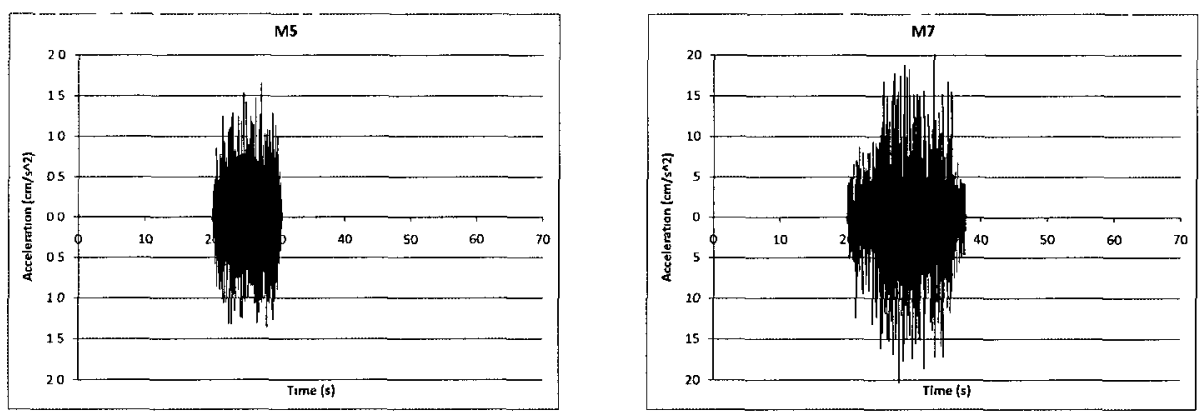

Figure 17: Simulated acceleration time series of $M 5$ and $M 7$ earthquakes at a distance of $100 \mathrm{~km}$ with $\varepsilon=0.1$ using a tapered boxcar window.

fractional stress drop cause changes in the simulated acceleration time series, which were simulated using a tapered boxcar window. The Fourier acceleration spectrum, the psuedo acceleration spectrum and the psuedo velocity spectrum for $M 5$ and $M 7$ earthquakes, as shown in Figures 19, 20 and 21, display the expected behaviour. The lower frequency portion of the spectra are scaled according to the $\varepsilon$ value, with the higher frequency spectra oscillating around the average values of the complete stress drop, $\varepsilon=1$.

In the input file for EXSIM_Beta, the option is added to use fractional stress drop as a 

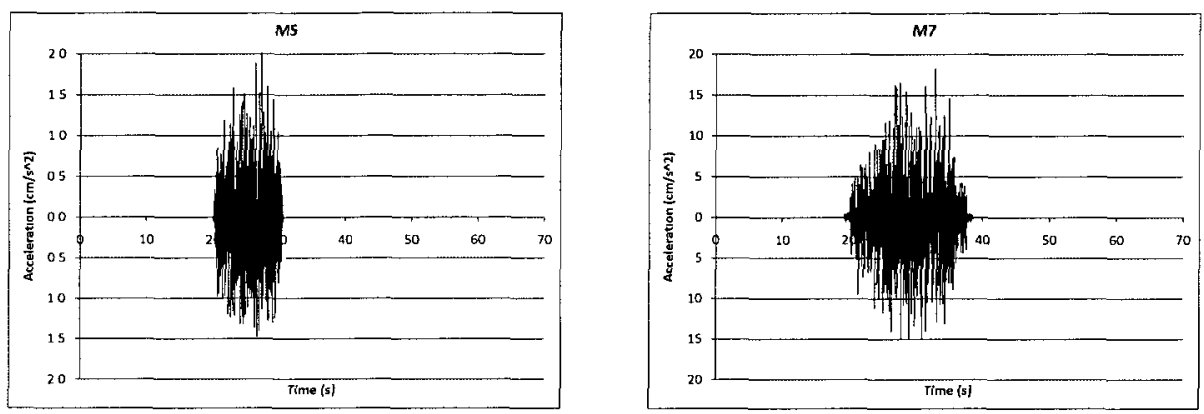

Figure 18: Simulated acceleration time series of $M 5$ and $M 7$ earthquakes at a distance of $100 \mathrm{~km}$ with $\varepsilon=1.3$ using a tapered boxcar window.
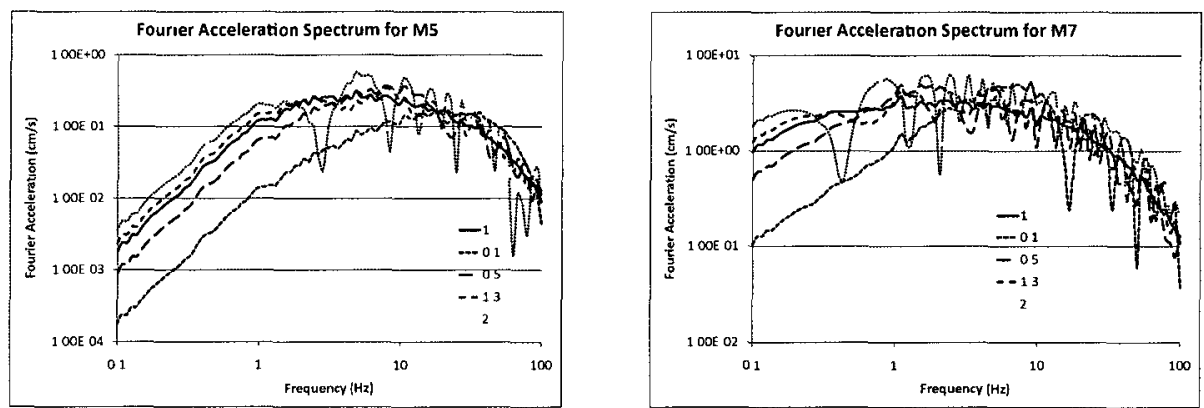

Figure 19: Simulated Fourier acceleration spectrum of $M 5$ and $M 7$ earthquakes at a distance of $100 \mathrm{~km}$ for different values of $\varepsilon$.
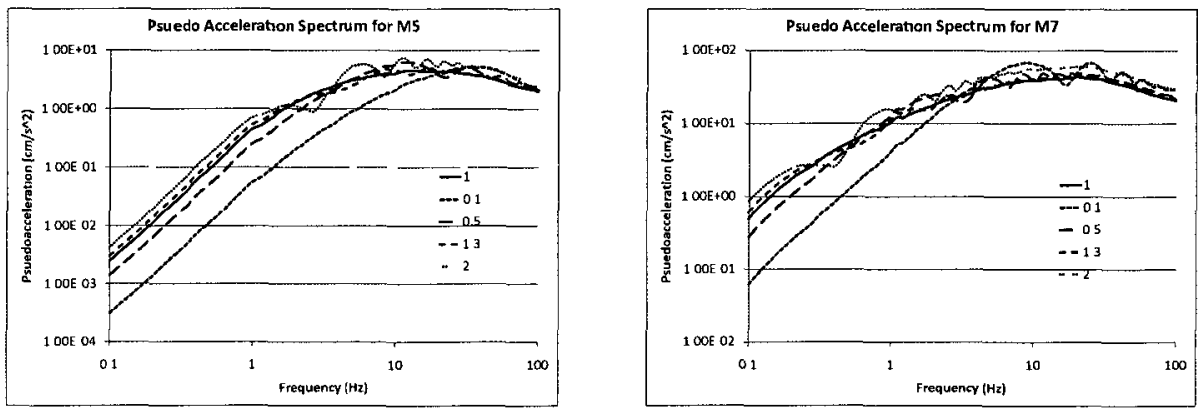

Figure 20: Simulated psuedo acceleration spectrum of $M 5$ and $M 7$ earthquakes at a distance of $100 \mathrm{~km}$ for different values of $\varepsilon$. 

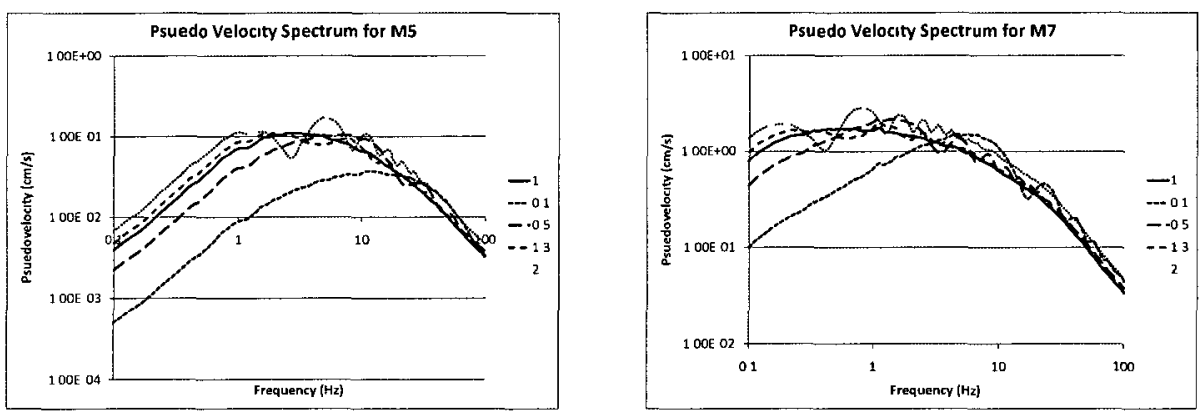

Figure 21: Simulated psuedo velocity spectrum of $M 5$ and $M 7$ earthquakes at a distance of $100 \mathrm{~km}$ for different values of $\varepsilon$.

low-frequency taper. The previous low-frequency taper option can now take on three values: 0 for Boore's taper, 1 for Motazedian's low-frequency coefficient, and 2 for fractional stress drop. There must be a value for the low-frequency parameter. If Boore's taper is selected, the low-frequency parameter is disregarded, but still is read in by the program. If Motazedian's low-frequency coefficient is selected it takes the value of the low-frequency parameter. If fractional stress drop is selected, $\varepsilon$ takes the value of the low-frequency parameter. 


\section{Chapter 5}

\section{Stress Drop and Low-Frequency Values}

As shown in the previous sections, the stress drop is an important parameter in determining the level of high-frequency ground motion. Therefore, it is important to have a good idea of what stress drop values are needed to reproduce accurate levels of ground motion from previous known and recorded earthquakes. The stress drop values from stochastic modelling should be compared to theoretical values or the values found from other methods, in order to determine if it should be adjusted for the purpose of predictive stochastic modelling.

The high frequency-portion of an earthquake spectrum is generally the important part for engineering purposes. This is the portion to which most low- to medium-rise buildings respond, and are most likely to be damaged. However, buildings in high-density urban areas are commonly being built taller, which shifts their natural frequency of vibration to lower frequencies. This has caused a greater concern for the accuracy of predictive earthquake modelling at low frequencies.

Since stochastic earthquake modelling is based on the fact that high-frequency ground motion is essentially random (Hanks, 1979; McGuire and Hanks 1980; Hanks and McGuire, 1981), predicting the low-frequency portion of the spectrum using this technique has been problematic. Recently, different ways have been incorporated in stochastic modelling to control the level of low-frequency earthquake spectra, which has been tested and compared with the previously measured values from the Pacific Earthquake Engineering 
Research Center (PEER) Next Generation of Ground-Motion Attenuation Models (NGA) database.

The values, that determine the accuracy of our model are the residuals at each frequency. The residual is defined as (Motazedian and Atkinson, 2005):

$$
\text { residual }=\log (\text { observed } P S A)-\log (\text { predicted } P S A)
$$

Calculation of this value at each frequency helps to determine if the predicted value is satisfactory and which frequency range is accurate for each simulation. Due to the random nature of earthquake events, it is not uncommon to have large residuals for any given simulation; therefore, the main focus is the average residuals determined for each earthquake.

\subsection{General Residual Analysis}

In finding the best value for the stress drop and the low-frequency parameter values for an earthquake using the stochastic model, several steps were involved. For each earthquake, all the recordings were looked at separately. Using the earthquake parameters, as described in detail in Appendices A and B, several simulations were run for each site. The stress drop was calculated first, since it was determined to be the most important parameter.

To find this value, two simulations were run as a starting point. The first run set the lower limit of the stress drop at $1 \mathrm{bar}$, and the second set the higher limit as 1,000 bars. The residuals were determined for all frequencies, and the sum of the residuals above $1 \mathrm{~Hz}$ was calculated. If the sum of the residuals was less than 0 for the first run, or greater than 0 for the second run, an error was produced as these values are probable limits of the stress drop of an earthquake (Aki, 1967, Brune 1970, Boore et al., 2010).

Using a bisection method, a simulation was run using a stress drop value of the average of the higher and lower limits. If the sum of the residuals above $1 \mathrm{~Hz}$ was above 0 , the 
stress drop value was taken as the lower stress drop limit; and, if the residual sum was less than 0 , the higher limit was taken as the value. This was repeated until a satisfactory value for the stress drop was found for the individual site.

If a low-frequency coefficient or fractional stress drop value were being used, they were set to 0.0 and 1.0 respectively, in the determination of the stress drop. After the value for the stress drop was found, the sum of residuals for frequencies below $1 \mathrm{~Hz}$ was calculated. If this value was below 0 , the lower limit low-frequency coefficient or fractional stress drop value was decreased. If the sum of the low-frequency residuals was above 0 , the higher limit low-frequency coefficient or fractional stress drop value was increased. The bisection method was then preformed for determining the most accurate low-frequency coefficient or fractional stress drop value.

After a satisfactory value for the low-frequency coefficient was found, three sums of the residuals were taken: 1) the sum of all the residuals over the entire frequency range, 2) the sum of residuals of frequencies above $1 \mathrm{~Hz}$, and 3) the sum of residuals below $1 \mathrm{~Hz}$. Each of the residual sums was determined to be either above, below, or within an acceptable limit, giving way to 27 possible outcomes.

Tables 7,8 and 9 show the different possibilities if the total residual was above, below, and within the limit, respectively, and the steps taken if that outcome was reached. After the appropriate step was applied, another simulation was run with those values as the limits for the corresponding value; and, another bisection method was applied. This would be repeated until either all residual limits were within their acceptable limits, i.e. the values for the stress drop and low-frequency coefficient were the same as the previous simulation, or there had been 20 iterations.

To do this quickly and efficiently, a program was developed using FORTRAN (Appendix D). This program would go through these methods, calling EXSIM_Beta when a simulation needed to be run. Using the output from EXSIM_Beta, this program was able to calculate the residuals and follow the processes from Tables 7, 8, and 9. The spectrum 


\begin{tabular}{|c|c|c|c|c|}
\hline \multirow{2}{*}{\multicolumn{2}{|c|}{ Total Residuals $>0$}} & \multicolumn{3}{|c|}{ Residuals above $1 \mathrm{~Hz}$} \\
\hline & & $>0$ & $=0$ & $<0$ \\
\hline \multirow{3}{*}{ Residualsbelow $1 \mathrm{~Hz}$} & $>0$ & increase $\sigma$ & decrease LFC & increase LFC \\
\hline & $=0$ & increase $\sigma$ & not possible & not possible \\
\hline & $<0$ & increase $\sigma$ & not possible & not possible \\
\hline
\end{tabular}

Table 7: Steps taken when the total residual sum is above the acceptable limit and the residuals above and below $1 \mathrm{~Hz}$ fall into each category

\begin{tabular}{|c|c|c|c|c|}
\hline \multirow{2}{*}{\multicolumn{2}{|c|}{ Total Residuals $<0$}} & \multicolumn{3}{|c|}{ Residuals above $1 \mathrm{~Hz}$} \\
\hline & & $>0$ & $=0$ & $<0$ \\
\hline \multirow{3}{*}{ Residualsbelow $1 \mathrm{~Hz}$} & $>0$ & not possible & not possible & decrease $\sigma$ \\
\hline & $=0$ & not possible & not possible & decrease $\sigma$ \\
\hline & $<0$ & increase $\sigma$ & increase LFC & decrease $\sigma$ \\
\hline
\end{tabular}

Table 8: Steps taken when the total residual sum is below the acceptable limit and the residuals above and below $1 \mathrm{~Hz}$ fall into each category

\begin{tabular}{|c|c|c|c|c|}
\cline { 3 - 5 } \multicolumn{2}{c}{} & \multicolumn{4}{c|}{ Residuals above $1 \mathrm{~Hz}$} \\
\cline { 3 - 6 } & & $>0$ & $=0$ & $<0$ \\
\hline \multirow{3}{*}{ Residualsbelow $1 \mathrm{~Hz}$} & $>0$ & not possible & increase LFC & decrease $\sigma$ \\
\cline { 2 - 6 } & $=0$ & increase $\sigma$ & accurate values found & decrease $\sigma$ \\
\cline { 2 - 6 } & $<0$ & increase $\sigma$ & decrease LFC & not possible \\
\hline
\end{tabular}

Table 9: Steps taken when the total residual sum is within the acceptable limit and the residuals above and below $1 \mathrm{~Hz}$ fall into each category 
above $10 \mathrm{~Hz}$ is strongly dependent on the $\kappa$ filter and anelastic attenuation, which may differ from region to region or even at different sites within a region. These higher frequencies were not considered when determining the stress drop value. When satisfactory stress drop values and low-frequency coefficients were found for each site, the program would output those values along with a summary of the steps taken, in order to ensure the values were correct.

Using the stress drop values and low-frequency coefficients for each site, the average for each earthquake was determined. These values are shown in Table 10, which provides the results for three different methods: Boore's taper, Motazedian's low-frequency coefficient, and fractional stress drop. It also includes the static stress drop values found in the NGA database. If no static stress drop value was shown in the database, one was calculated using the defined fault plane used in the simulations and the same formula used in the NGA database (Kanamori and Anderson, 1975):

$$
\sigma=\frac{7 M_{0}}{16 * 10^{6}\left(A * 10^{10} / \pi\right)^{1.5}}
$$

where $M_{0}$ is the seismic moment and $A$ is the total area of the fault. Figures 22, 23, and 24 plot the residuals versus distance at $0.2,1.0,10.0$, and $50.0 \mathrm{~Hz}$ and show the average residual for each frequency calculated for the quantitative results of Boore's taper, Motazedian's low-frequency coefficient, and fractional stress drop, respectively.

Figure 25 displays the average residuals at each frequency for the different methods used. Using fractional stress drop gave the most accurate simulated ground motions over the entire range of frequencies. Figure 26 shows the average residual versus frequency for each of the different methods for the Northridgel earthquake. It is a good comparison of how the different methods affect the high-frequency and low-frequency spectra. 


\begin{tabular}{|c|c|c|c|c|c|c|c|c|c|c|c|}
\hline \multirow{2}{*}{ Earthquake Name } & \multicolumn{1}{|c|}{ Boore's taper } & \multicolumn{4}{|c|}{ Motazedian's taper } & \multicolumn{3}{|c|}{ Fractional stress drop } & \multirow{2}{*}{ Static Stress Drop (bars) } \\
\cline { 2 - 12 } & $\sigma$ & $\sigma_{s t d}$ & $\sigma$ & $\sigma_{s t d}$ & LFC & $L F C_{\text {std }}$ & $\sigma$ & $\sigma_{\text {std }}$ & $\varepsilon$ & $\varepsilon_{\text {std }}$ & \\
\hline Anza 02 & 111.7 & 104.0 & 120.3 & 94.0 & -0.50 & 0.93 & 140.0 & 109.7 & 0.95 & 0.52 & $5.3^{*}$ \\
\hline Coalinga 01 & 77.6 & 45.4 & 206.0 & 126.0 & 0.60 & 0.28 & 75.0 & 44.6 & 1.44 & 0.24 & $40.6^{\dagger}$ \\
\hline Coalinga 02 & 246.6 & 219.9 & 237.0 & 130.9 & 0.02 & 0.61 & 226.9 & 209.8 & 1.27 & 0.49 & $25.5^{*}$ \\
\hline Hector Mine & 48.8 & 30.6 & 63.4 & 38.6 & 0.00 & 1.10 & 43.3 & 25.4 & 1.20 & 0.44 & $36.2^{\dagger}$ \\
\hline Landers & 41.9 & 48.6 & 59.2 & 74.3 & -0.84 & 1.66 & 37.9 & 48.6 & 0.87 & 0.44 & $64.5^{\dagger}$ \\
\hline Northridge 01 & 119.3 & 85.2 & 117.0 & 156.0 & -0.73 & 1.59 & 96.9 & 71.0 & 1.02 & 0.68 & $33.0^{\dagger}$ \\
\hline Northridge 04 & 47.6 & 18.7 & 52.3 & 28.0 & -1.12 & 0.51 & 39.9 & 15.2 & 0.71 & 0.25 & $57.4^{*}$ \\
\hline Northridge 05 & 55.5 & 36.6 & 143.3 & 119.1 & 0.47 & 0.27 & 58.4 & 40.7 & 1.52 & 0.32 & $36.3^{*}$ \\
\hline Northridge 06 & 126.2 & 76.5 & 94.2 & 103.7 & -0.82 & 0.89 & 130.2 & 79.8 & 0.78 & 0.47 & $35.8^{*}$ \\
\hline San Fernando & 90.3 & 175.3 & 50.2 & 62.3 & -1.09 & 1.59 & 48.4 & 62.4 & 1.00 & 0.75 & $24.5^{\dagger}$ \\
\hline
\end{tabular}

Table 10: Results for finding stress drop $(\sigma)$ in bars, low frequency coefficient (LFC) and fractional stress drop $(\varepsilon)$, along with the standard deviation of each $\left(\sigma_{s t d}, L F C_{s t d}\right.$, and $\left.\varepsilon_{s t d}\right)$ using a general residual analysis approach. 

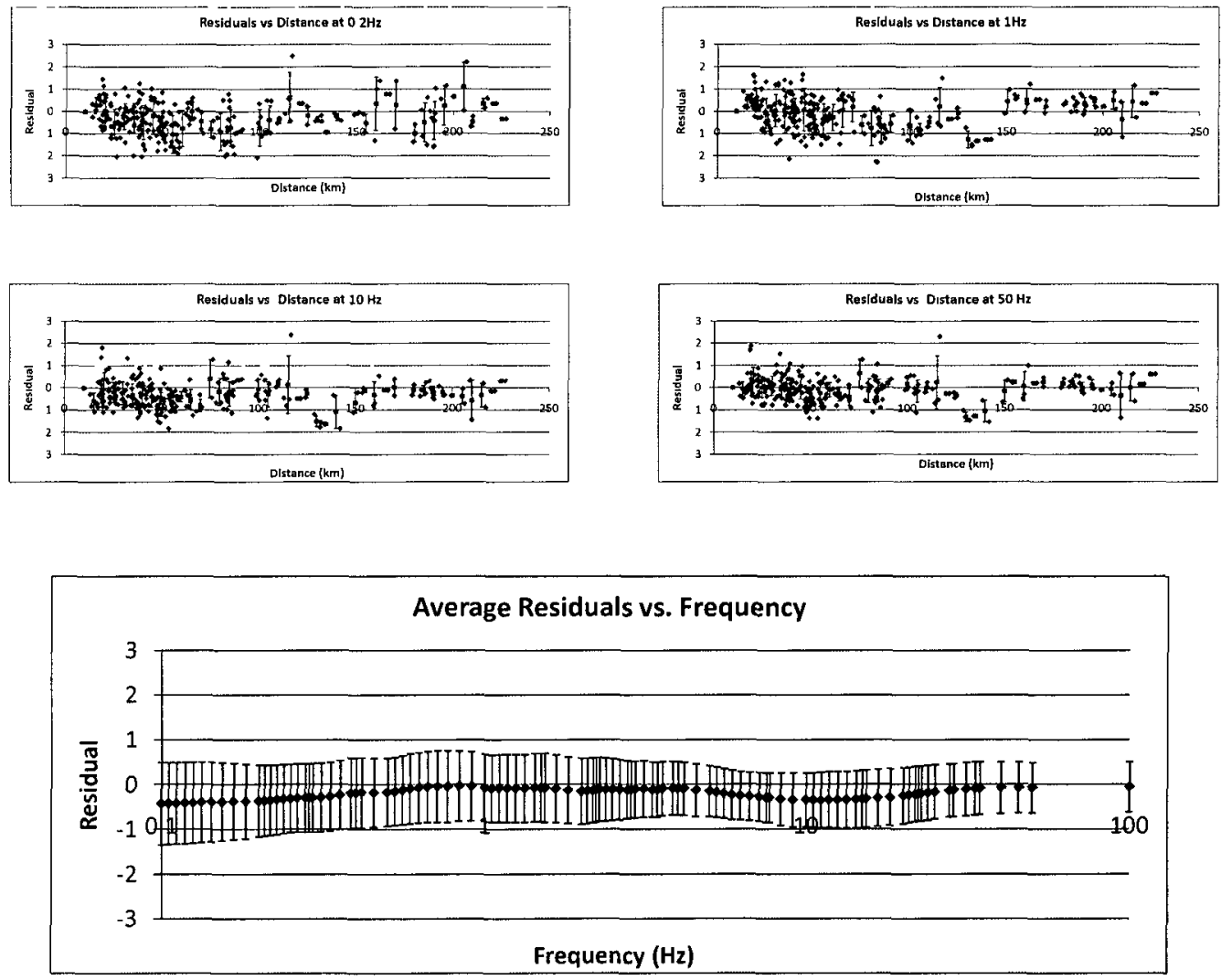

Figure 22: Residuals for all earthquakes using the general residual analysis results for Boore's taper along with the average residual for each distance every $5 \mathrm{~km}$; and, the average residual at each frequency. 

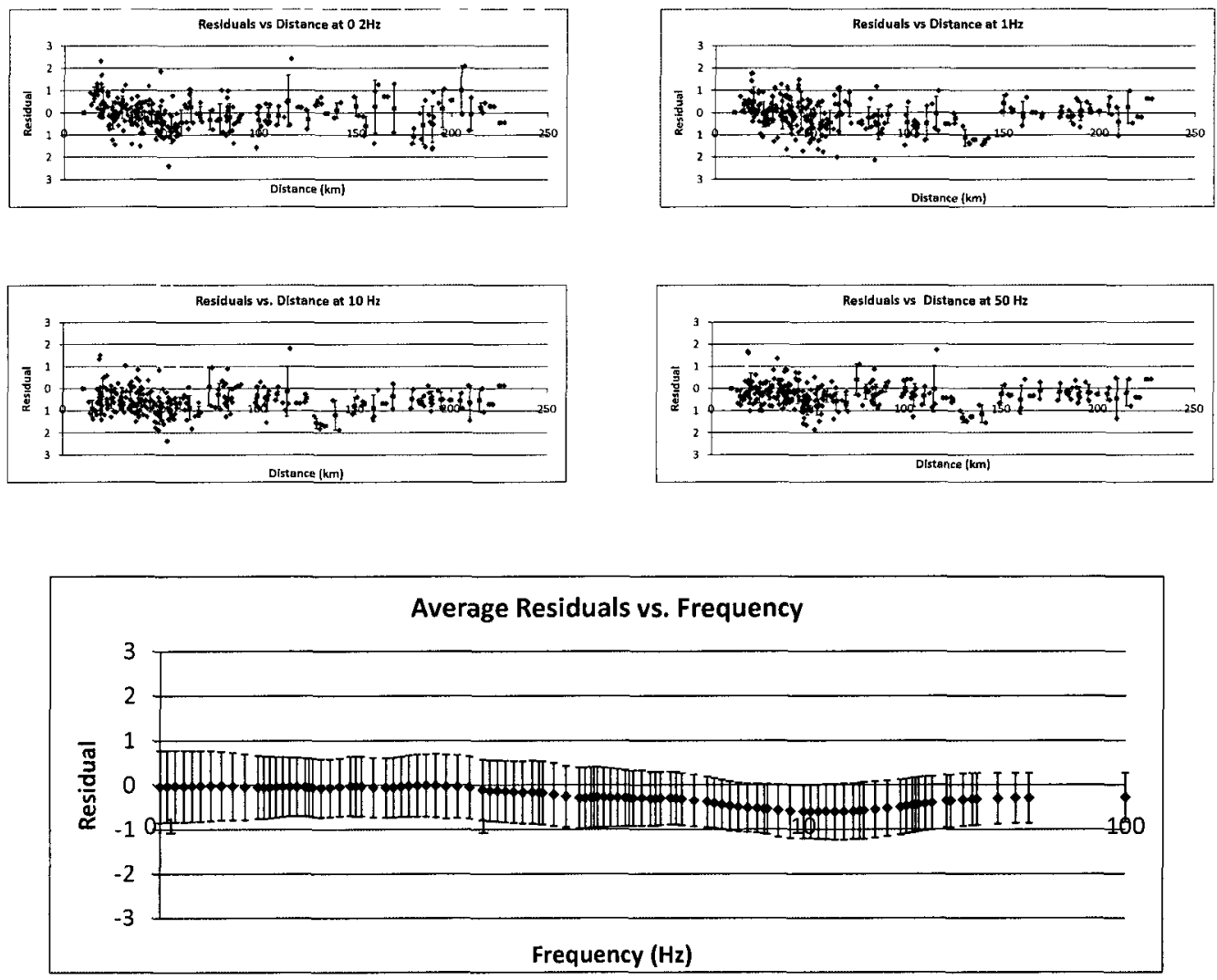

Figure 23: Residuals for all earthquakes using the general residual analysis results for Motazedian's low-frequency coefficient along with the average residual for each distance every $5 \mathrm{~km}$; and, the average residual at each frequency. 

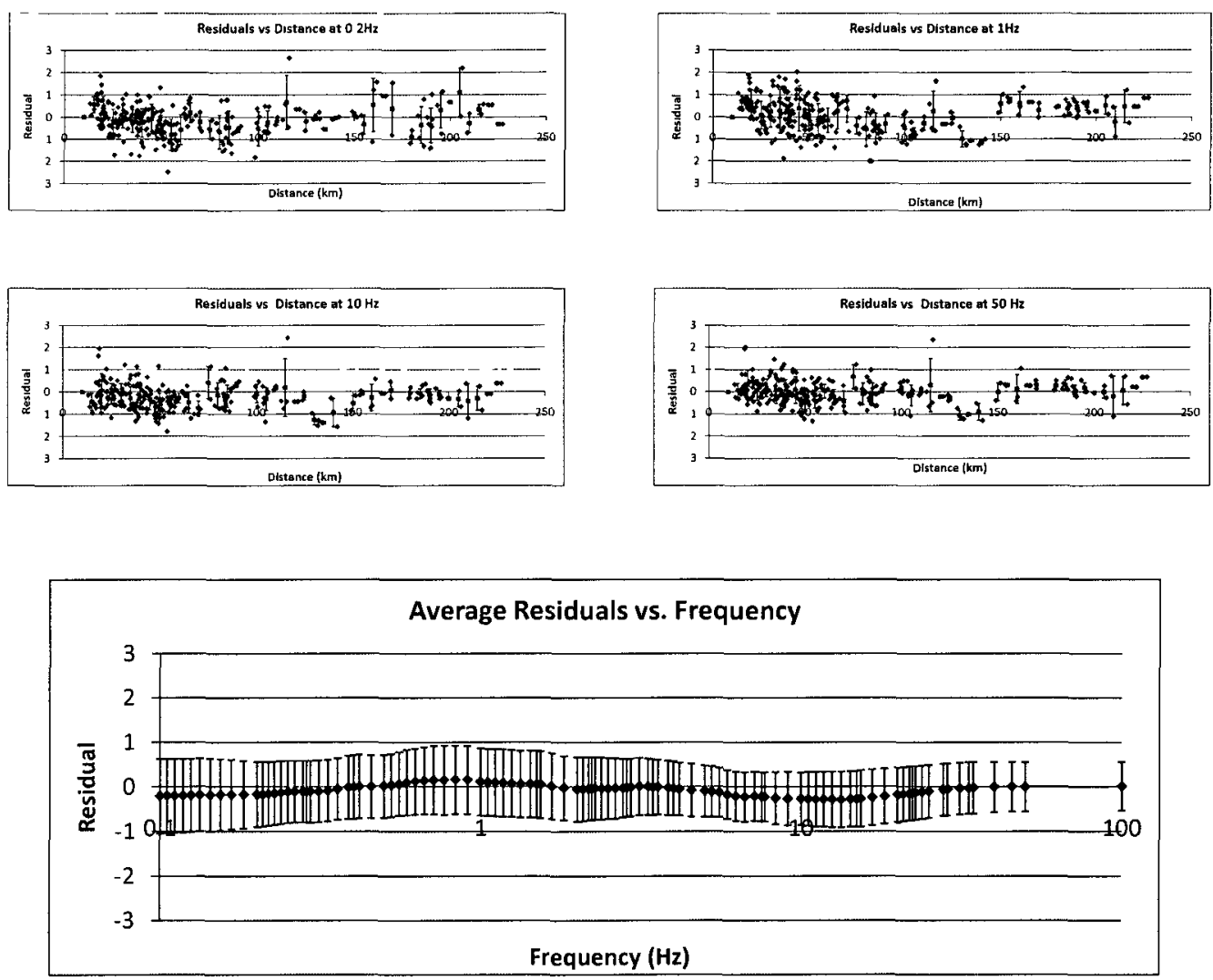

Figure 24: Residuals for all earthquakes using the general residual analysis results for fractional stress drop along with the average residual for each distance every $5 \mathrm{~km}$; and, the average residual at each frequency. 


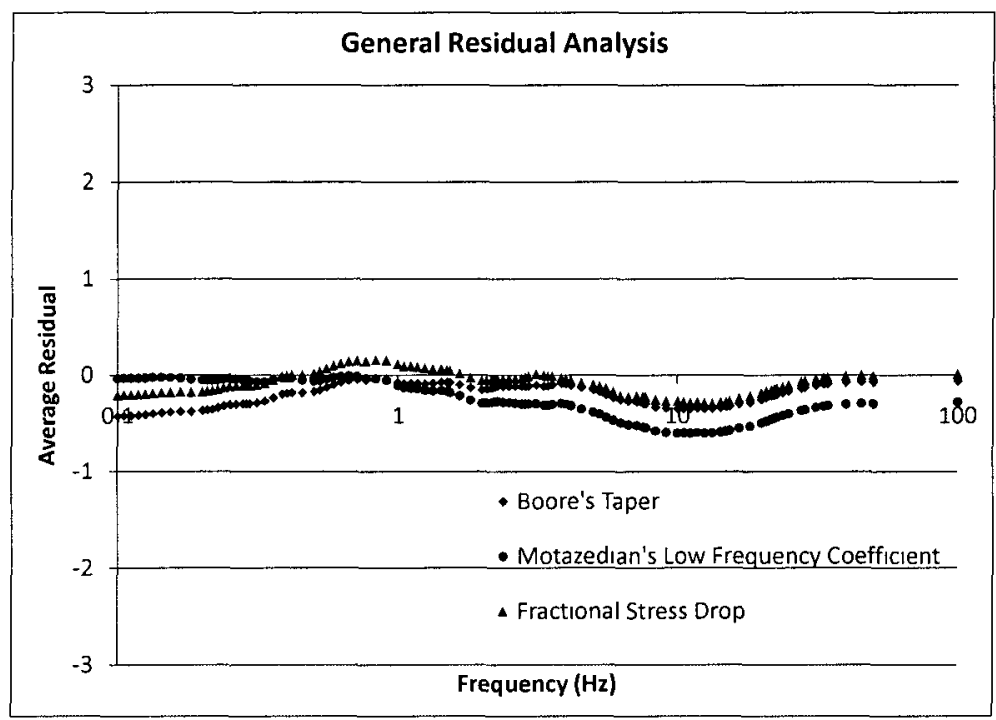

Figure 25: Average residuals at each frequency for each method using general residual analysis.

\subsection{Earthquake Specific Residual Analysis}

Plotting the residuals from the quantitative measurements, as shown in Figure 26, which displays the average residuals for the Northridge1 earthquake using Motazedian's lowfrequency coefficient, we can see how the average residuals behave for each earthquake. Although the averages of the residuals may be within the acceptable limits, the distribution of residuals is not an ideal distribution. To get better average residuals, the stress drop values and low-frequency coefficients were adjusted manually, until any trends in the residuals were either minimized or preferably eliminated. The resulting residuals are plotted in Figure 27, as a comparison for the Northridge 1 earthquake, which shows the residual trends have been reduced.

Table 11 shows the resulting values of the stress drop and low-frequency coefficient for each of the different earthquakes found using a qualitative approach. These results give a 


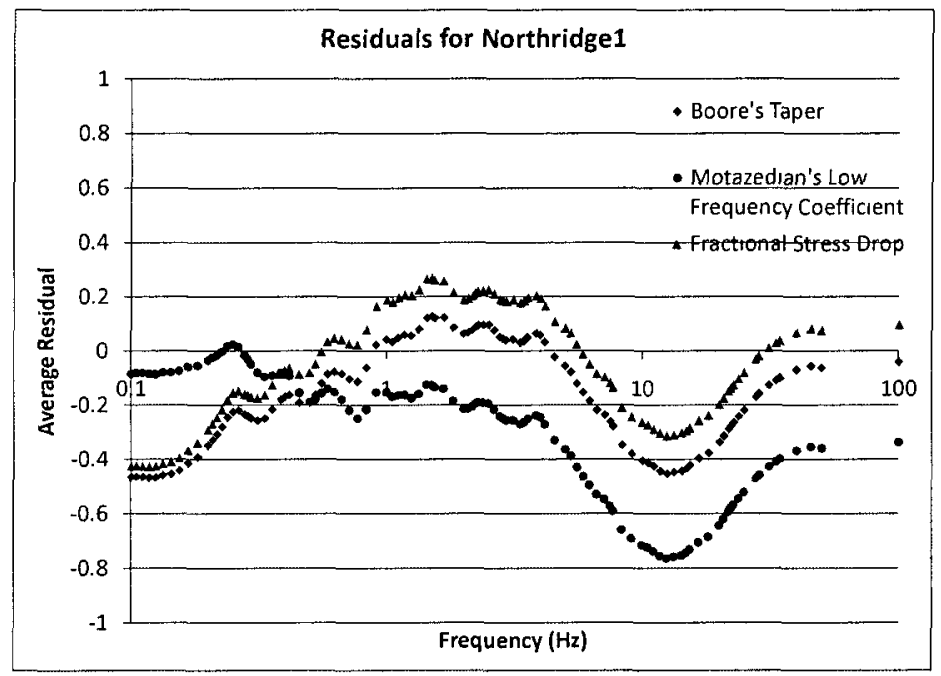

Figure 26: Residuals for the Northridge1 earthquake for Boore's taper, Motazedian's lowfrequency coefficient and fractional stress drop using a general residual analysis approach.
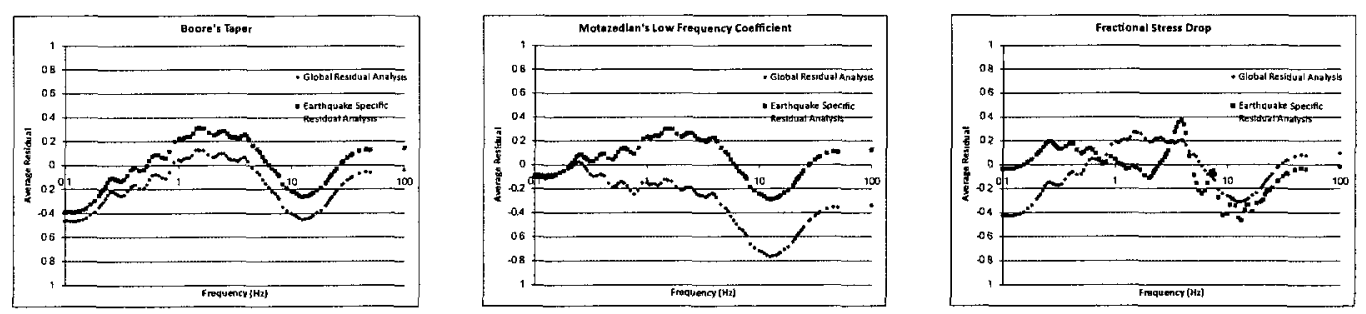

Figure 27: Residuals for the Northridge 1 earthquake for Boore's taper, Motazedian's lowfrequency coefficient and fractional stress drop using an earthquake specific residual analysis approach with the residuals from the general residual approach included. 


\begin{tabular}{|c|c|c|c|c|c|c|}
\hline \multirow{2}{*}{ Earthquake Name } & Boore's taper & \multicolumn{2}{|c|}{ Motazedian's taper } & \multicolumn{2}{|c|}{ Fractional stress drop } & \multirow{2}{*}{ Static Stress Drop (bars) } \\
\cline { 2 - 6 } & $\sigma$ & $\sigma$ & LFC & $\sigma$ & $\varepsilon$ & \\
\hline Anza 02 & 100 & 100 & -0.75 & 90 & 0.70 & $5.3^{*}$ \\
\hline Coalinga 01 & 95 & 120 & 0.20 & 80 & 1.00 & $40.6^{\dagger}$ \\
\hline Coalinga 02 & 175 & 200 & 0.20 & 200 & 1.25 & $25.5^{*}$ \\
\hline Hector Mine & 45 & 90 & 0.60 & 50 & 1.30 & $36.2^{\dagger}$ \\
\hline Landers & 45 & 30 & -0.80 & 40.0 & 0.60 & $64.5^{\dagger}$ \\
\hline Northridge 01 & 90 & 70 & -0.40 & 100 & 0.60 & $33.0^{\dagger}$ \\
\hline Northridge 04 & 25 & 15 & -1.00 & 30 & 0.50 & $57.4^{*}$ \\
\hline Northridge 05 & 70 & 110 & 0.60 & 60 & 1.00 & $36.3^{*}$ \\
\hline Northridge 06 & 90 & 90 & -0.20 & 100 & 0.75 & $35.8^{*}$ \\
\hline San Fernando & 35 & 30 & -0.30 & 35 & 0.90 & $24.5^{\dagger}$ \\
\hline
\end{tabular}

Table 11: Results for finding stress drop $(\sigma)$ in bars, low frequency coefficient (LFC) and fractional stress drop $(\varepsilon)$ using an earthquake specific residual analysis approach. 
better overall residual plot, as shown in Figures 28,29, and 30, when compared to the total residuals found when using the quantitative approach, as in Figures 22, 23, and 24. The small negative trend has been reduced, and the average of the residuals of each frequency is close to zero.

Figure 31 displays the average residual at each frequency of the different methods using specific earthquake residual analysis. Using this type of approach, the simulations are more accurate in both the high-frequency and low-frequency ranges when compared to the average residuals calculated from a general residual analysis (Figure 25). 

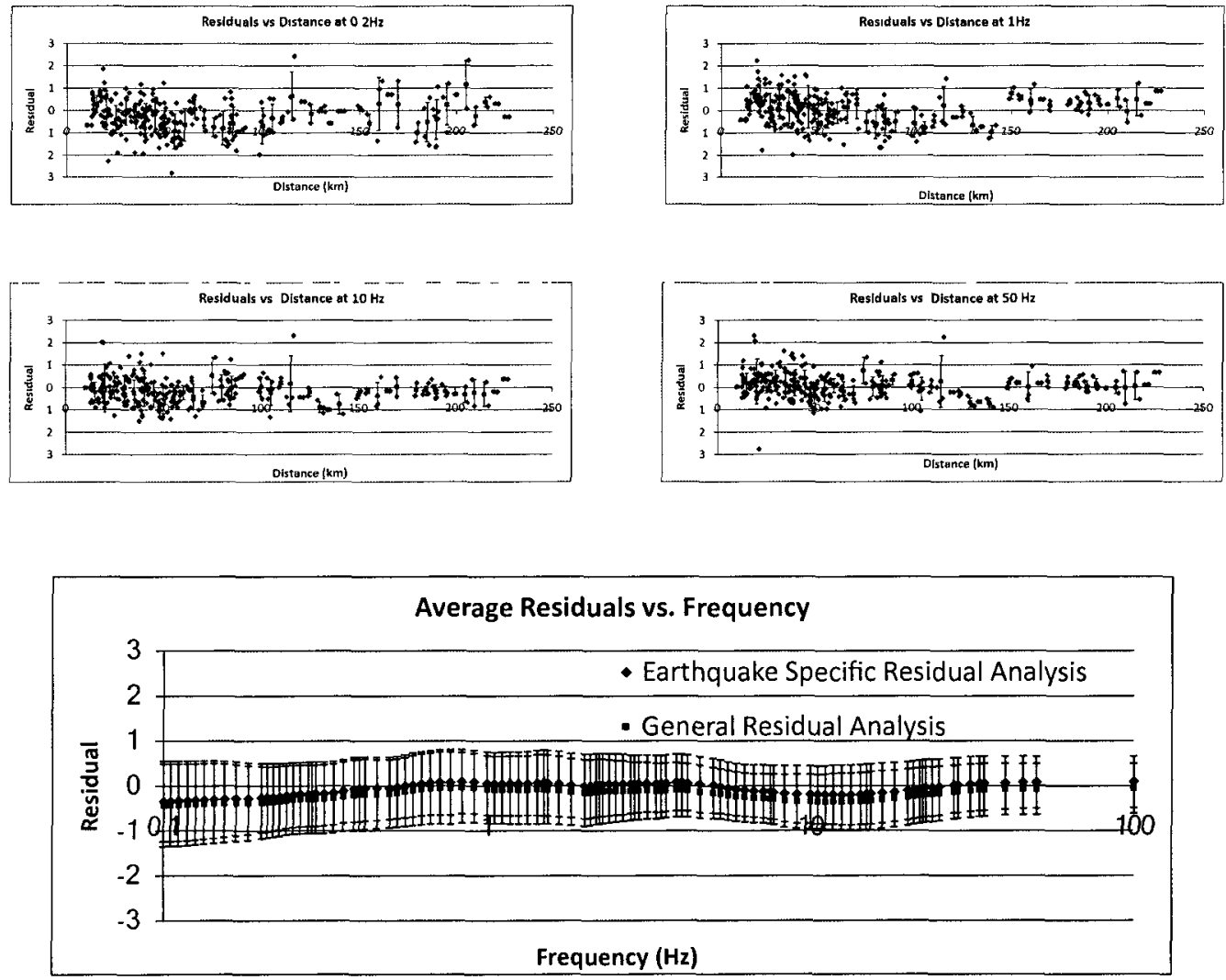

Figure 28: Residuals for all earthquakes using the earthquake specific residual analysis results for Boore's taper along with the average residual for each distance every 5 $\mathrm{km}$; and, the average residual at each frequency, including the average residual from the general residual analysis. 

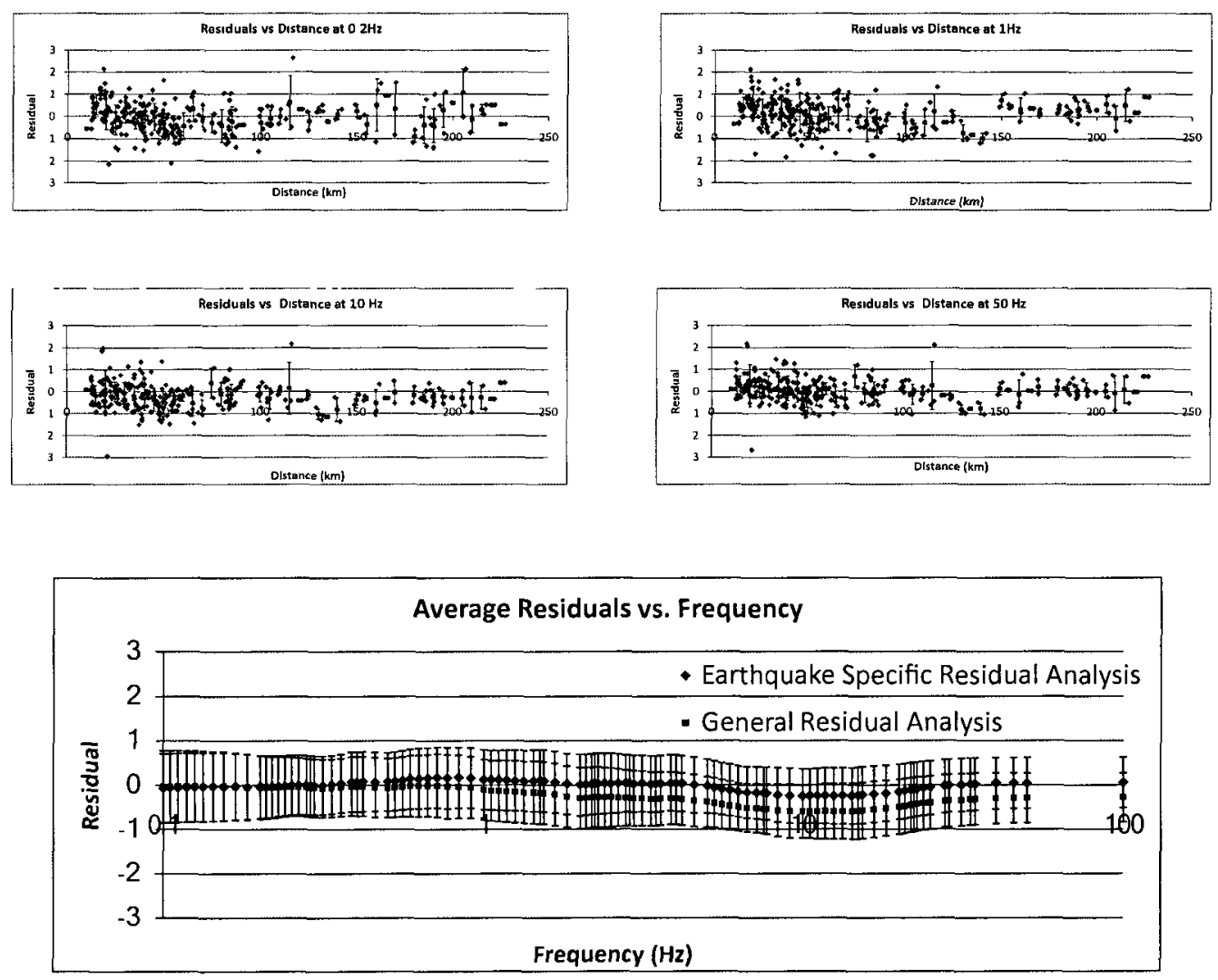

Figure 29: Residuals for all earthquakes using the earthquake specific residual analysis results for Motazedian's low-frequency coefficient along with the average residual for each distance every $5 \mathrm{~km}$; and, the average residual at each frequency, including the average residual from the general residual analysis. 

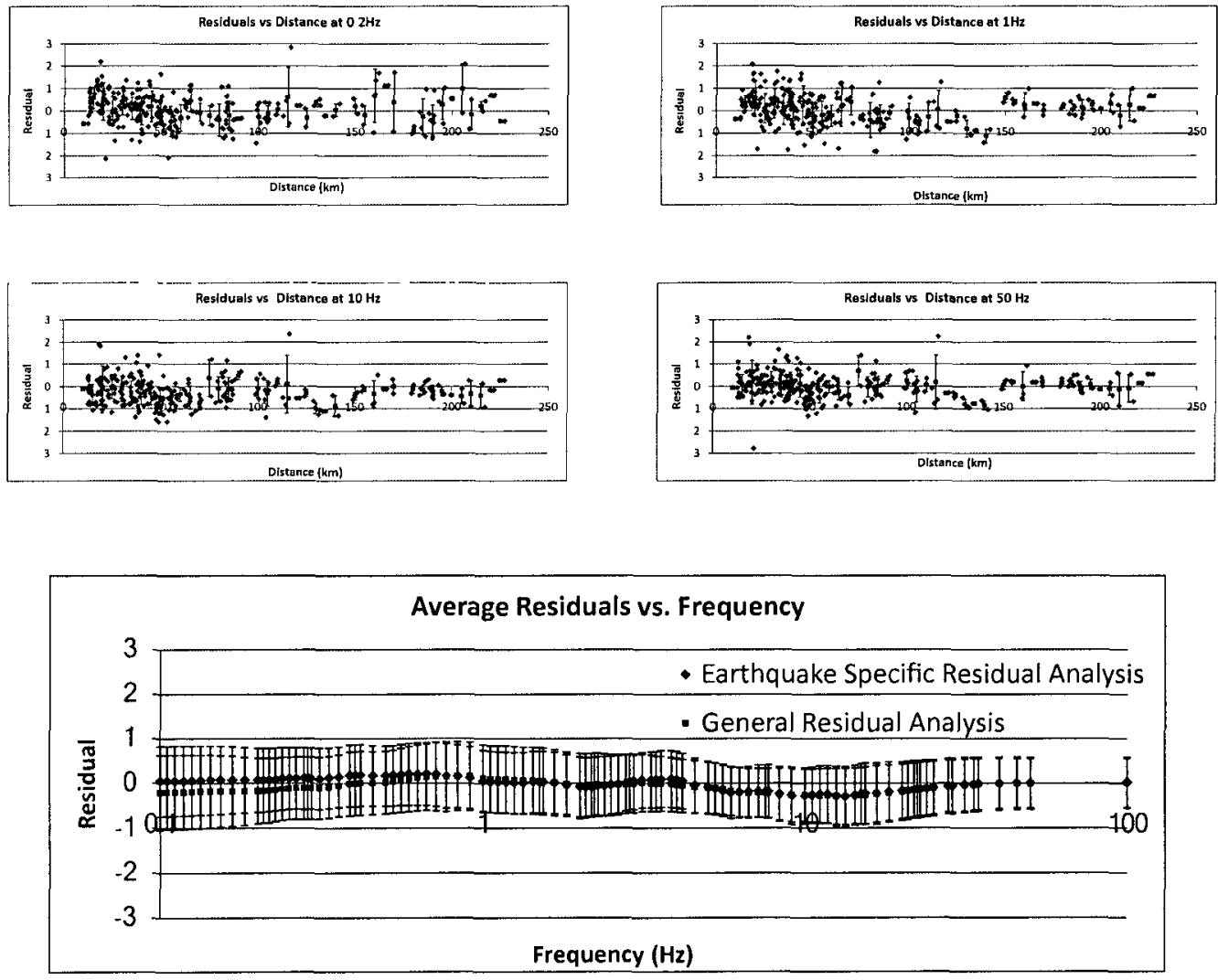

Figure 30: Residuals for all earthquakes using the earthquake specific residual analysis results for fractional stress drop along with the average residual for each distance every $5 \mathrm{~km}$; and, the average residual at each frequency, including the average residual from the general residual analysis. 


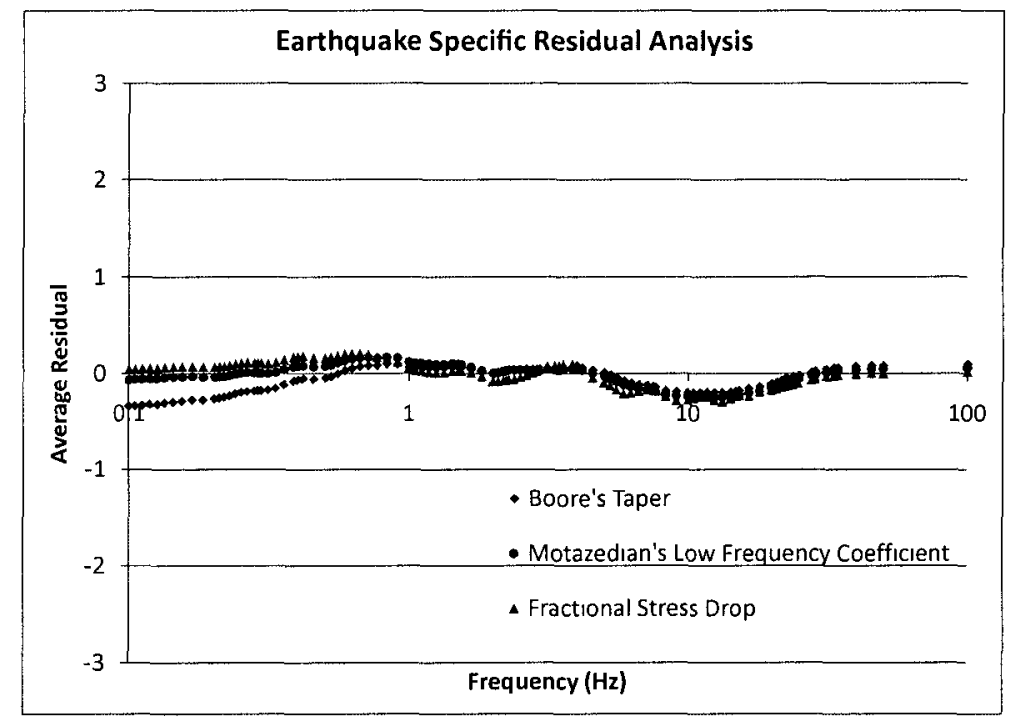

Figure 31: Average residuals at each frequency for each method using specific earthquake residual analysis. 


\section{Chapter 6}

\section{Conclusion and Future Studies}

There are many different ways of modelling an earthquake, using different types of methods. Stochastic modelling techniques insert a random component for high-frequency ground motions along with a target spectral amplitude, in order to reproduce an acceleration time series similar to one that is recorded. There are even different types of stochastic modelling, inculding stochastic point-source modelling as utilized by the program SMSIM (Boore, 1983 and 1996), and stochastic finite-fault modelling used by FINSIM (Beresnev and Atkinson, 1998a), EXSIM (Motazedian and Atkinson, 2005) and EXSIM_Beta, a modified version of EXSIM introduced in this research. These different stochastic modelling techniques are widely used to model past earthquakes and predict future ground motions.

Modelling an earthquake is not an easy task, because ground motion is affected in many different ways, which is taken into account with different parameters. Source parameters describe the physical process of an earthquake rupture and include the fault geometry and the size of an earthquake. Regional path effects occur when the ground motions travel to a recording site. The properties of the material and the region through which a wave propagates affects the signal duration and the signal amplitude from geometrical spreading and anelastic attenuation. Local geological settings have specific effects on ground motions and are modelled with frequency dependent site effects. All these effects, along with scaling of low frequencies, are taken into account when using a stochastic modelling technique. 
EXSIM (Motazedian and Atkinson, 2005) is a useful tool, applying stochastic finitefault modelling to simulate an acceleration time series. It was modified by Boore (2009), in order to produce results that agree more closely with SMSIM (Boore, 1983; Boore, 1996), which is a stochastic point-source modelling technique. EXSIM produces accurate results modelling recorded earthquakes, but there were a few changes still to be made. Adding the option of fault segments has provided better control of fault geometry for large earthquakes, which can have several rupture areas that are not well represented by a single rectangular fault. Adding the option of having fractional stress drop or including Motazedian's low-frequency coefficient has provided a way of scaling the low-frequency spectrum of an earthquake. This is a new feature introduced to stochastic finite-fault modelling.

The low-frequency scaling techniques used by the stochastic finite-fault modelling of EXSIM_Beta provide greater control and the ability to adjust the amplitude of the lowfrequency spectrum. As shown previously, these simulation techniques are able to calibrate low-frequency ground motions without adjusting the high-frequency ground motions. This is a useful and important tool for stochastic modelling which has previously performed inadequately in simulating low-frequency ground motions. The low-frequency parameters added to the program, which take the form of either Motazedian's low-frequency coefficient or fractional stress drop, produce a simple method of adjusting the low-frequency spectrum. Boore's method of tapering low frequencies provides better control of low-frequency amplitudes without any additional parameters added.

Source codes for EXSIM_Beta, EXSIM and FINSIM are available upon request. The SMSIM programs used in this research were obtained from the personal website of David Boore at http://www.daveboore.com/software_online.htm last accessed on July 29th, 2011. 


\subsection{Suggested Future Studies}

In the studied earthquakes, the parameters were determined using each of the lowfrequency scaling methods. Each method gave reasonable results for all earthquakes but some of the scaling methods were better suited for a specific earthquake. One example of this occurred with the 1999 Hector Mine earthquake. Boore's taper and fractional stress drop produced stochastic stress drop values close to the static stress drop provided, and Motazedian's low-frequency coefficient method resulted in a stress drop two to three times the amount of the static stress drop. Earthquakes similar to this one could produce results favouring Boore's taper and fractional stress drop as the preferred low-frequency scaling methods.

Apply these modelling techniques to a large number of earthquakes will make it easier to determine the best use of each method for other earthquakes. This study used only a limited number of earthquakes to determine the parameters, in order to show that these simulation techniques can accurately reproduce recorded ground motions. Using a large database of earthquakes and determining the parameters for a greater number of different earthquakes will provide a better understanding of the usefulness of each method. It will also allow for a better study of any dependency of low-frequency scaling values on magnitudes and focal mechanisms.

Determining a trend in the change of the stochastic stress drop level after a lowfrequency parameter has been adjusted may eliminate the need to calibrate the higher frequency spectra a second time. This may provide a much quicker and easier way to calibrate the parameters for any earthquake. Figures 10 and 11 display the change in the higher frequency portion of the spectra when a low-frequency parameter took on different values.

An investigation of the possible dependency of low-frequency scaling values on the 
depth of earthquakes may provide a starting point for calibrating the stochastic modelling parameters. For example, there may be a correlation between the value of the lowfrequency parameter and earthquakes that occur in a subduction zone, compared to those in an intraplate setting. This may also correlate to a dependency of the low-frequency scaling values on regions, such as western or eastern North America. Discovering these or any other correlations will reduce the calculation time needed for calibrating stochastic modelling parameters.

The low-frequency scaling methods presented in this thesis are new to stochastic modelling and should be compared with other types of earthquake modelling. These lowfrequency scaling methods in stochastic modelling should be compared to empirical and theoretical Green function modelling as well as deterministic modelling. These comparisons will provide an understanding of how stochastic modelling using low-frequency scaling methods performs against other types of earthquake modelling. This type of comparison will help to determine which type of earthquake modelling is best suited for a specific purpose. 
Appendix A

\section{Table of Western North America Parameters}




\begin{tabular}{cc}
\hline Parameter & Value \\
\hline$\kappa$ filter & 0.035 \\
$\beta$ & $3.7 \mathrm{~km} / \mathrm{s}$ \\
$\rho$ & $2.8 \mathrm{~g} / \mathrm{cm}^{3}$ \\
$\gamma(\mathrm{vrup} / \beta)$ & 0.8 \\
$\Delta \mathrm{t}$ & $0.002 \mathrm{sec}$ \\
Geometrical Spreading: $R^{b}$ & $0 \mathrm{~km}<R \leq 40 \mathrm{~km} \mathrm{~b}=-1.0$ \\
& $40 \mathrm{~km}<R \leq 130 \mathrm{~km} \mathrm{~b}=-0.5$ \\
& $130 \mathrm{~km}<R b=-0.5$ \\
Trilinear Duration: & $1 \mathrm{~km}<R \leq 100 \mathrm{~km} \mathrm{~d}=0.1$ \\
& $100 \mathrm{~km}<R \leq 500 \mathrm{~km} \mathrm{~d}=0.1$ \\
Anelastic attenuation: $\mathrm{Q}(\mathrm{f})$ & $500 \mathrm{~km}<R \mathrm{~d}_{3}=0.1$ \\
Window & max $\left(1000.0,180 f^{0.45}\right)$ \\
\% dampening & Saragoni-Hart \\
Pulsing percent & $5 \%$ \\
Crustal Amplification & $100 \%$ \\
Site Amplification & Table 4 \\
\hline
\end{tabular}

Table 12: A summary of the parameters used for simulating the earthquakes in Western North America. 
Appendix B

\section{Earthquake Specific Parameters}




\begin{tabular}{cc}
\hline \multicolumn{2}{c}{ Anza 02 } \\
\hline Parameter & Value \\
\hline Magnitude & 4.92 \\
Width of Fault $(\mathrm{km})$ & 4.8 \\
Depth to Fault $(\mathrm{km})$ & 15.2 \\
Fault Dip & $78^{\circ}$ \\
Beginning of Fault & \\
Latitude & $33.54^{\circ}$ \\
Longitude & $-116.48^{\circ}$ \\
End of Fault & \\
Latitude & $33.507^{\circ}$ \\
Longitude & $-116.52^{\circ}$ \\
Subfault Length $(\mathrm{km})$ & 0.93 \\
Subfault Width $(\mathrm{km})$ & 0.93 \\
Hypocentre location $(i, j)$ & $(1,5)$ \\
\hline
\end{tabular}

Table 13: A summary of the parameters used for simulating the Anza 02 earthquake. 


\begin{tabular}{cc}
\hline \multicolumn{2}{c}{ Coalinga 01 } \\
\hline Parameter & Value \\
\hline Magnitude & 6.36 \\
Width of Fault $(\mathrm{km})$ & 11 \\
Depth to Fault $(\mathrm{km})$ & 3.4 \\
Fault Dip & $30^{\circ}$ \\
Beginning of Fault & \\
Latitude & $36.29^{\circ}$ \\
Longitude & $-120.35^{\circ}$ \\
End of Fault & \\
Latitude & $36.18^{\circ}$ \\
Longitude & $-120.23^{\circ}$ \\
Subfault Length $(\mathrm{km})$ & 3.5 \\
Subfault Width $(\mathrm{km})$ & 3.5 \\
Hypocentre location $(i, j)$ & $(3,2)$ \\
\hline
\end{tabular}

Table 14: A summary of the parameters used for simulating the Coalinga 01 earthquake. 


\begin{tabular}{cc}
\hline \multicolumn{2}{c}{ Coalinga 02 } \\
\hline Parameter & Value \\
\hline Magnitude & 5.09 \\
Width of Fault $(\mathrm{km})$ & 3.3 \\
Depth to Fault $(\mathrm{km})$ & 12 \\
Fault Dip & $44^{\circ}$ \\
Beginning of Fault & \\
Latitude & $36.25^{\circ}$ \\
Longitude & $-120.3^{\circ}$ \\
End of Fault & \\
Latitude & $36.27^{\circ}$ \\
Longitude & $-120.33^{\circ}$ \\
Subfault Length $(\mathrm{km})$ & 1.09 \\
Subfault Width $(\mathrm{km})$ & 1.09 \\
Hypocentre location $(i, j)$ & $(1,1)$ \\
\hline
\end{tabular}

Table 15: A summary of the parameters used for simulating the Coalinga 02 earthquake. 


\begin{tabular}{cc}
\hline \multicolumn{2}{c}{ Hector Mine } \\
\hline Parameter & Value \\
\hline Magnitude & 7.13 \\
Width of Fault $(\mathrm{km})$ & 16.2 \\
Depth to Fault $(\mathrm{km})$ & 0 \\
Fault Dip & $77^{\circ}$ \\
Beginning of Fault & \\
Latitude & $34.2^{\circ}$ \\
Longitude & $-116.16^{\circ}$ \\
End of Fault & \\
Latitude & $34.74^{\circ}$ \\
Longitude & $-116.52^{\circ}$ \\
Subfault Length $(\mathrm{km})$ & 7.11 \\
Subfault Width $(\mathrm{km})$ & 7.11 \\
Hypocentre location $(i, j)$ & $(5,7)$ \\
\hline
\end{tabular}

Table 16: A summary of the parameters used for simulating the Hector Mine earthquake. 


\begin{tabular}{cc}
\hline \multicolumn{2}{c}{ Landers } \\
\hline Parameter & Value \\
\hline Magnitude & 7.28 \\
Width of Fault $(\mathrm{km})$ & 15.0 \\
Depth to Fault $(\mathrm{km})$ & 0 \\
Fault Dip & $90^{\circ}$ \\
Beginning of Fault & \\
Latitude & $33.5^{\circ}$ \\
Longitude & $-116.38^{\circ}$ \\
End of Fault & \\
Latitude & $34.08^{\circ}$ \\
Longitude & $-116.34^{\circ}$ \\
Subfault Length $(\mathrm{km})$ & 8.17 \\
Subfault Width $(\mathrm{km})$ & 8.17 \\
Hypocentre location $(i, j)$ & $(9,1)$ \\
\hline
\end{tabular}

Table 17: A summary of the parameters used for simulating the Landers earthquake. 


\begin{tabular}{cc}
\hline \multicolumn{2}{c}{ Northridge 01 } \\
\hline Parameter & Value \\
\hline Magnitude & 6.69 \\
Width of Fault $(\mathrm{km})$ & 24.0 \\
Depth to Fault $(\mathrm{km})$ & 5 \\
Fault Dip & $40^{\circ}$ \\
Beginning of Fault & \\
Latitude & $34.39^{\circ}$ \\
Longitude & $-118.6^{\circ}$ \\
End of Fault & \\
Latitude & $34.30^{\circ}$ \\
Longitude & $-118.43^{\circ}$ \\
Subfault Length $(\mathrm{km})$ & 4.74 \\
Subfault Width $(\mathrm{km})$ & 4.74 \\
Hypocentre location $(i, j)$ & $(4,3)$ \\
\hline
\end{tabular}

Table 18: A summary of the parameters used for simulating the Northridge 01 earthquake. 


\begin{tabular}{cc}
\hline \multicolumn{2}{c}{ Northridge 04 } \\
\hline Parameter & Value \\
\hline Magnitude & 5.93 \\
Width of Fault $(\mathrm{km})$ & 5.9 \\
Depth to Fault $(\mathrm{km})$ & 9.8 \\
Fault Dip & $40^{\circ}$ \\
Beginning of Fault & \\
Latitude & $34.26^{\circ}$ \\
Longitude & $-118.65^{\circ}$ \\
End of Fault & \\
Latitude & $34.33^{\circ}$ \\
Longitude & $-118.70^{\circ}$ \\
Subfault Length $(\mathrm{km})$ & 2.36 \\
Subfault Width $(\mathrm{km})$ & 2.36 \\
Hypocentre location $(i, j)$ & $(2,1)$ \\
\hline
\end{tabular}

Table 19: A summary of the parameters used for simulating the Northridge 04 earthquake. 


\begin{tabular}{cc}
\hline \multicolumn{2}{c}{ Northridge 05 } \\
\hline Parameter & Value \\
\hline Magnitude & 5.13 \\
Width of Fault $(\mathrm{km})$ & 3.1 \\
Depth to Fault $(\mathrm{km})$ & 11.3 \\
Fault Dip & $40^{\circ}$ \\
Beginning of Fault & \\
Latitude & $34.36^{\circ}$ \\
Longitude & $-118.66^{\circ}$ \\
End of Fault & \\
Latitude & $34.38^{\circ}$ \\
Longitude & $-118.69^{\circ}$ \\
Subfault Length $(\mathrm{km})$ & 1.13 \\
Subfault Width $(\mathrm{km})$ & 1.13 \\
Hypocentre location $(i, j)$ & $(1,1)$ \\
\hline
\end{tabular}

Table 20: A summary of the parameters used for simulating the Northridge 05 earthquake. 


\begin{tabular}{cc}
\hline \multicolumn{2}{c}{ Northridge 06 } \\
\hline Parameter & Value \\
\hline Magnitude & 5.28 \\
Width of Fault $(\mathrm{km})$ & 3.7 \\
Depth to Fault $(\mathrm{km})$ & 13.1 \\
Fault Dip & $40^{\circ}$ \\
Beginning of Fault & \\
Latitude & $34.22^{\circ}$ \\
Longitude & $-118.45^{\circ}$ \\
End of Fault & \\
Latitude & $34.24^{\circ}$ \\
Longitude & $-118.49^{\circ}$ \\
Subfault Length $(\mathrm{km})$ & 1.29 \\
Subfault Width $(\mathrm{km})$ & 1.29 \\
Hypocentre location $(i, j)$ & $(4,5)$ \\
\hline
\end{tabular}

Table 21: A summary of the parameters used for simulating the Northridge 06 earthquake. 


\begin{tabular}{cc}
\hline \multicolumn{2}{c}{ San Fernando } \\
\hline Parameter & Value \\
\hline Magnitude & 6.61 \\
Width of Fault $(\mathrm{km})$ & 27.4 \\
Depth to Fault $(\mathrm{km})$ & 0 \\
Fault Dip & $50^{\circ}$ \\
Beginning of Fault & \\
Latitude & $34.3^{\circ}$ \\
Longitude & $-118.17^{\circ}$ \\
End of Fault & \\
Latitude & $34.34^{\circ}$ \\
Longitude & $-118.34^{\circ}$ \\
Subfault Length $(\mathrm{km})$ & 4.41 \\
Subfault Width $(\mathrm{km})$ & 4.41 \\
Hypocentre location $(i, j)$ & $(2,3)$ \\
\hline
\end{tabular}

Table 22: A summary of the parameters used for simulating the San Fernando earthquake. 
Appendix C

Table of Generic Earthquake Parameters 


\begin{tabular}{|c|c|c|c|c|}
\hline Parameter & \multicolumn{4}{|c|}{ Value } \\
\hline Stress drop & \multicolumn{4}{|c|}{60 bars } \\
\hline$\kappa$ filter & \multicolumn{4}{|c|}{0.005} \\
\hline$\beta$ & \multicolumn{4}{|c|}{$3.7 \mathrm{~km} / \mathrm{s}$} \\
\hline$\rho$ & \multicolumn{4}{|c|}{$2.8 \mathrm{~g} / \mathrm{cm}^{3}$} \\
\hline$\gamma(\operatorname{vrup} / \beta)$ & \multicolumn{4}{|c|}{0.8} \\
\hline$\Delta \mathrm{t}$ & \multicolumn{4}{|c|}{$0.002 \mathrm{sec}$} \\
\hline Geometrical Spreading: $R^{b}$ & \multicolumn{4}{|c|}{$\begin{array}{c}70 \mathrm{~km}<R \leq 140 \mathrm{~km} b=0.2 \\
140 \mathrm{~km}<R b=-0.5\end{array}$} \\
\hline Trilinear Duration: & $\begin{array}{r}0 \\
70 k\end{array}$ & $\begin{array}{l}k m<R \\
m<R \leq \\
130 \mathrm{~km}\end{array}$ & $\begin{array}{l}\leq 70 \mathrm{~km} \\
<130 \mathrm{~km} \\
<R d_{3}\end{array}$ & $\begin{array}{l}d_{1}=0.16 \\
d_{2}=-0.03 \\
=0.04\end{array}$ \\
\hline Anelastic attenuation & \multicolumn{4}{|c|}{$\max \left(1000.0,893 f^{032}\right)$} \\
\hline Window & \multicolumn{4}{|c|}{ Saragoni-Hart } \\
\hline$\%$ dampening & \multicolumn{4}{|c|}{$5 \%$} \\
\hline Pulsing percent & \multicolumn{4}{|c|}{$100 \%$} \\
\hline Crustal amplification & \multicolumn{4}{|c|}{ Table 4} \\
\hline Strike & \multicolumn{4}{|c|}{$122.0^{\circ}$} \\
\hline Dip & \multicolumn{4}{|c|}{$20.0^{\circ}$} \\
\hline Depth & \multicolumn{4}{|c|}{$0.0 \mathrm{~km}$} \\
\hline Parameter & $M 5$ & $M 6$ & $M 7$ & $M 8$ \\
\hline Fault Length $(\mathrm{km})$ & 4.17 & 13.18 & 41.69 & 131.83 \\
\hline Fault Width (km) & 3.89 & 8.13 & 16.98 & 35.48 \\
\hline Subfault Length (km) & 1 & 2.51 & 6.31 & 15.85 \\
\hline Subfault Width (km) & 1 & 2.51 & 6.31 & 15.85 \\
\hline
\end{tabular}

Table 23: The parameters used for simulating the generic earthquakes. The fault length and width were calculated using the magnitude relationships from Wells and Coppersmith (1994). 


\section{Appendix D}

\section{FORTRAN Program for Calibrating Stress Drop and a Low Frequency Parameter}

program LowFreqCalibration

c program for calibrating the low frequency coefficient of EXSIM

logical f_exist, resNotGood

character InputFileName*30,f_stem $* 30$,fresp $1 * 30$, fresp $2 * 30$

character ampFile*30, siteAmp*30, flfc*30

character NEHRP

real dl_in, dw_in

real freq_out $(10)$

integer nHypocenters

integer i0(10), j0(10)

real maxPulsingDistance(10), distanceToSegOrigin

real slipWeights $(200,200)$

dimension siteLocation $(300,2)$

real segFaultLat(10,2), segFaultLon(10,2), segFaultStrike(10),

: segFaultDip(10), segFaultLength(10)

real $\mathrm{dl}(10)$, avgdl

dimension segnl(10)

integer begOfFaultSegment, hypoFaultSeg(10)

integer numberOfFaults, faultSeg(200)

real subFaultRadius(10), riseTime_original(10)

integer endOfFaultSegment(10), faultIntersection(10,2)

real siteLat, siteLon, low $F$ 
real psaT(105,500),psa(105)

integer count, numAmpFile, numSiteAmp

integer earthID, stationNumber

real pga(500), pgv(500), pgd(500)

real simpsa(105), freq(500), psaResidual(105,3) !1 is lowStress, 2 is highStress 3 is midStress

real avgRes(105), avgResLow, avgResHigh, avgResMid

integer freqCount, error, stressCount

real avgLFC, lowFreqCoeff, psaRes(105), freqC

real hishStressLimit, lowStressLimit, highLFC, lowLFC

real lowFreqLimit, highFreqLimit, totalAvgRes

integer residualError, doWhileCount

character datx $* 8$, time_start*10, time_stop*10

$$
\begin{aligned}
& \text { nu_ctl }=99 \\
& \text { ioInput }=\text { nu_ctl }-1 \\
& \text { ioStation }=\text { nu_ctl }-2 \\
& \text { ioSiteAmp }=\text { nu_ctl }-3 \\
& \text { ioCrustalAmp }=\text { nu_ctl }-4 \\
& \text { ioAmpFile }=\text { nu_ctl }-5 \\
& \text { ioSiteAmp }=\text { nu_ctl }-6 \\
& \text { ioSim }=\text { nu_ctl }-7 \\
& \text { ioOutput }=\text { nu_ctl }-8 \\
& \text { ioAvgRes }=\text { nu_ctl }-9
\end{aligned}
$$

call DATE_AND_TIME( datx, time_start )

f_exist $=$.false .

do while (.not. f_exist)

InputFileName $=$, ,

write $(*$, '(a)')

: ' Enter name of input parameter file '//

: '(cr = EXSIMLFC.in):

read(*, '(a)') InputFileName

if (InputFileName( $1: 4)$.eq. ', $)$

: InputFileName $=$ 'EXSIM LFC.in'

call trim_c(InputFileName, nc_f_in)

inquire(file=InputFileName(1:nc_f_in), exist=f_exist)

if (.not. f_exist) then

write(*,'(a)')' ******** FILE DOES NOT EXIST *******,

end if

end do 
print *, ' Control file: '//InputFileName(1:nc_f_in)

open(unit=nu_ctl, file=InputFileName(1:nc_f_in), status='unknown')

c Read (and echo) the input parameters that are common to all grid points. call getInputParameters(nu_ctl,

: FaultStrike,FaultDip,h,

: numberOfFaults,

: segFaultLat, segFaultLon,

: segFaultStrike, segFaultDip,

: segFaultLength,

: FaultLat,FaultLon,

: siteLocation,numberOfSites,isitecoordflag,

: FaultLength, FaultWidth,

: dl_in, dw_in, nl, nw, nsubs,

: segnl, endOfFaultSegment,

: $\mathrm{y}$,

: i0_in,j0_in,nHypocenters,

: dt, beta, rho, amag,

: stress,iKapa,fmax,

: Q0,eta, Qmin,

: rg1,rg2,pow1,pow2,pow3,

: rmin,rd1,rd2,durmin,b1,b2,b3, ! trilinear duration parameters

: iwind,

: flocut, nslope,

: nfreq,freq1,freq2,

: nfout, freq_out,

: f_stem,

: namp1,fresp1, namp2,fresp2,

: iseed, nsims,damp,

: Islipweight, slipWeights,

: iRow,jColumn,pulsingPercent,

: iPapaFlag,PapaGama,PapaNu,PapaT0,PapaPeak,

: Low_frequencies_taper,

: lowFreqCoeff) !Motazedian Feb, 10029

close(nu_ctl)

$\mathrm{v}=\mathrm{y} *$ beta

call getFreq(nfreq,freq) 


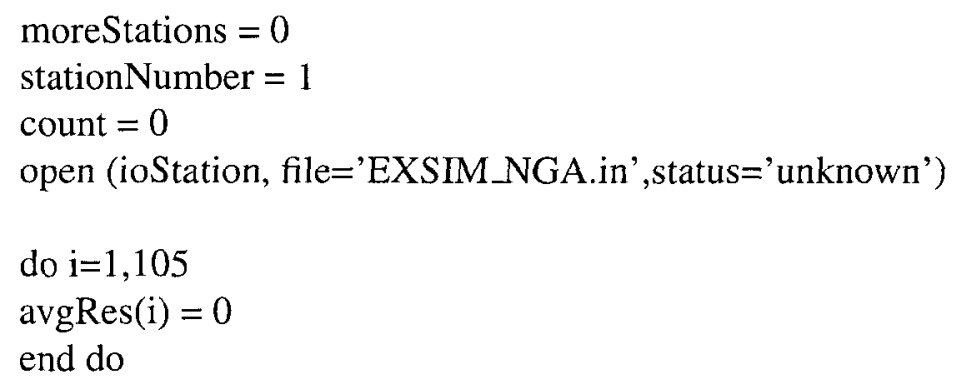

do while (moreStations .eq. 0)

call readEarthquakeStationParameters (ioStation, pga, pgv, : pgd, NEHRP, siteLat, siteLon, lowF, psaT, : earthID, siteAmp, moreStations, numSiteAmp,

: ioAmpFile, ioSiteAmp, stationNumber)

if (morestations.eq.0.and.(NEHRP.eq.'C'.or.NEHRP

:.eq.'B'.or.NEHRP.eq.'A')) then

count $=$ count +1

error $=0$

fffc = 'LFC_output_000.out'

write(flfc(12:14),'(i3.3)') stationNumber

open (ioOutput, file=flfc, status='unknown')

call getFreqPSA(psaT,psa,stationNumber)

ccc This is where the bisection method was, now need to include stress and LowFreqCoeff freqC $=1.0$

lowFreqLimit $=1.0$

highFreqLimit $=100.0$

highStressLimit $=999.0$

lowStressLimit $=1.0$

lowFreqCoeff $=0.0$

stress $=$ bestStress $($ highStressLimit,lowStressLimit,freqC,

: highFreqLimit,error,ioInput,FaultStrike,FaultDip,h,

: numberOfFaults,segFaultLat, 
: segFaultLon,segFaultStrike, segFaultDip,segFaultLength,FaultLat, : FaultLon,siteLat, siteLon,FaultLength, FaultWidth,dl_in, dw_in,

: nl,nw,nsubs,segnl, endOfFaultSegment,y,i0_in,j0_in,nHypocenters, : dt, beta, rho, amag,iKapa,fmax,Q0,eta, Qmin,

: rg1,rg2,pow1, pow2,pow3,rmin,rd1,rd2,durmin,b1,b2,b3,iwind,

: flocut, nslope,nfreq,freq1,freq2,nfout, freq_out,f_stem,

: namp1,fresp1, numSiteAmp, siteAmp,iseed, nsims,damp,

: Islipweight, slipWeights, iRow,jColumn,pulsingPercent,

: iPapaFlag,PapaGama,PapaNu,PapaT0,PapaPeak,Low_frequencies_taper, : lowFreqCoeff, psaResidual,ioSim,simpsa,psa,freq,stationNumber,

: earthID)

resNotGood $=$. TRUE.

residualError $=0$

doWhileCount $=0$

do while(resNotGood.and.residualError.eq.0)

Create the name for the output files

f_stem = 'Output_000_000_00000_0000000'

write(f_stem(8:10),'(i3.3)') earthID

write(f_stem(12:14),'(i3.3)') stationNumber

write(f_stem(16:20),'(f5.1)') stress

write(f_stem(22:28),'(f7.4)') lowFreqCoeff

call trim_c(f_stem, nc_f_stem)

open (ioInput, file='EXSIM_MFIn.in',status='unknown')

call writeInputFile(ioInput,

: FaultStrike,FaultDip,h,

: numberOfFaults,

: segFaultLat, segFaultLon,

: segFaultStrike, segFaultDip,

: segFaultLength,

: FaultLat,FaultLon,

: siteLat, siteLon,

: FaultLength, FaultWidth,

: dl_in, dw_in, nl, nw, nsubs,

: segnl, endOfFaultSegment,

: $y$,

: i0_in,j0_in,nHypocenters,

: dt, beta, rho, amag,

: stress,iKapa,fmax,

: Q0,eta, Qmin, 
: rg1,rg2,pow1,pow2,pow3,

: rmin,rd1,rd2,durmin,b1,b2,b3, ! trilinear duration parameters

: iwind,

: flocut, nslope,

: nfreq,freq1,freq2,

: nfout, freq_out,

: f_stem,

: namp1,fresp1, numSiteAmp, siteAmp,

: iseed, nsims,damp,

: Islipweight, slipWeights,

: iRow,jColumn,pulsingPercent,

: iPapaFlag,PapaGama,PapaNu,PapaT0,PapaPeak,

: Low_frequencies_taper,

: lowFreqCoeff)

close(ioInput)

call system('EXSIM_Steve2.exe')

open(ioSim, file='simulatedParameters.out', status='unknown')

read $\left(\mathrm{ioSim},{ }^{*}\right)(\operatorname{simpsa}(\mathrm{j}), \mathrm{j}=1,105)$

close(ioSim)

do $i=1,105$

simpsa $(\mathrm{i})=\operatorname{simpsa}(\mathrm{i}) / 981.0$

end do

do $\mathrm{i}=1,105$

psaRes(i) $=\log ($ psa(i) $)-\log (\operatorname{simpsa}(\mathrm{i}))$

end do

avgResLow $=0$

freqCount $=0$

do $\mathrm{i}=1,105$

if (freq(i).le.lowFreqLimit) then

avgResLow $=$ avgResLow + psaRes(i)

freqCount $=$ freqCount +1

end if

end do

avgResLow $=\operatorname{avgResLow} /$ float $($ freqCount $)$

avgResHigh $=0$

freqCount $=0$ 
do $\mathrm{i}=1,105$

if (freq(i).le.highFreqLimit.and.freq(i).ge.lowFreqLimit) then

avgResHigh $=$ avgResHigh + psaRes(i)

freqCount $=$ freqCount +1

end if

end do

avgResHigh $=$ avgResHigh/float(freqCount)

totalAvgRes $=0$

do $\mathrm{i}=1,105$

totalAvgRes $=$ totalAvgRes + psaRes(i)

end do

totalAvgRes $=$ totalAvgRes $/ 105.0$

write (ioOutput, '('Low, High, Total Residuals: ",

: f7.4,1x,f7.4,1x,f7.4)') avgResLow, avgResHigh, totalAvgRes

write (ioOutput, '('Stress, LFC: ",f6.1,1x,f7.4)')

: stress, lowFreqCoeff

tempStress $=$ stress

tempLFC $=$ lowFreqCoeff

if(totalAvgRes.It.-0.05) then !totalAvgRes below limit

if(avgResHigh.lt.-0.01) then !high Residual below limit

if(avgResLow.lt.-0.1) then !low Residual below limit

write (ioOutput, '("Case: IA1")')

highStressLimit $=$ stress

lowStressLimit $=$ stress -100.0

if(lowStressLimit.lt.1.0) lowStresslimit $=1.0$

stress $=$ bestStress $($ highStressLimit,lowStressLimit,stress,freqC,

: highFreqLimit,error,ioInput,FaultStrike,FaultDip,h,

: numberOfFaults, segFaultLat,

: segFaultLon,segFaultStrike, segFaultDip,segFaultLength,FaultLat,

: FaultLon,siteLat, siteLon,FaultLength, FaultWidth,dl_in, dw_in,

: nl,nw,nsubs,segnl, endOfFaultSegment,y,i0_in,j0_in,nHypocenters,

: dt, beta, rho, amag,iKapa,fmax,Q0,eta, Qmin,

: rg1,rg2,pow1,pow2,pow3,rmin,rd1,rd2,durmin,b1,b2,b3,iwind,

: flocut, nslope,nfreq,freq1,freq2,nfout, freq_out,f_stem,

: namp1,fresp 1, numSiteAmp, siteAmp,iseed, nsims,damp,

: Islipweight, slipWeights,iRow,jColumn,pulsingPercent,

: iPapaFlag,PapaGama,PapaNu,PapaT0,PapaPeak,Low_frequencies_taper,

: lowFreqCoeff, psaResidual,ioSim,simpsa,psa,freq,stationNumber, 
: earthID)

else if(avgResLow.gt.0.1) then !Low Residual above limit

write (ioOutput, '("Case: IA2")')

highStressLimit $=$ stress

lowStressLimit $=$ stress -100.0

if(lowStressLimit.1t.1.0) lowStresslimit $=1.0$

stress $=$ bestStress $($ highStressLimit,lowStressLimit,stress,freqC,

: highFreqLimit,error,ioInput,FaultStrike,FaultDip,h,

: numberOfFaults,segFaultLat,

: segFaultLon,segFaultStrike, segFaultDip,segFaultLength,FaultLat,

: FaultLon,siteLat, siteLon,FaultLength, FaultWidth,dl_in, dw_in,

: nl,nw,nsubs,segnl, endOfFaultSegment,y,iO_in,j0_in,nHypocenters,

: dt, beta, rho, amag,iKapa,fmax,Q0,eta, Qmin,

: rg1,rg2,pow1,pow2,pow3,rmin,rd1,rd2,durmin,b1,b2,b3,iwind,

: flocut, nslope,nfreq,freq1,freq2,nfout, freq_out,f_stem,

: namp1,fresp1, numSiteAmp, siteAmp,iseed, nsims,damp,

: Islipweight, slipWeights,iRow,jColumn,pulsingPercent,

: iPapaFlag,PapaGama,PapaNu,PapaT0,PapaPeak,Low_frequencies_taper,

: lowFreqCoeff, psaResidual,ioSim,simpsa,psa,freq,stationNumber,

: earthID)

else !Low Residual within limits

write (ioOutput, '('Case: IA3")')

highStressLimit $=$ stress

lowStressLimit $=$ stress -100.0

if(lowStressLimit.lt.1) lowStresslimit $=1.0$

stress $=$ bestStress $($ highStressLimit,lowStressLimit,stress,freqC,

: highFreqLimit,error,ioInput,FaultStrike,FaultDip,h,

: numberOfFaults, segFaultLat,

: segFaultLon,segFaultStrike, segFaultDip,segFaultLength,FaultLat,

: FaultLon,siteLat, siteLon,FaultLength, FaultWidth,dl_in, dw_in,

: nl,nw,nsubs,segnl, endOfFaultSegment,y,i0_in,j0_in,nHypocenters,

: dt, beta, rho, amag,iKapa,fmax,Q0,eta, Qmin,

: rg1,rg2,pow1,pow2,pow3,rmin,rd1,rd2,durmin,b1,b2,b3,iwind,

: flocut, nslope,nfreq,freq1,freq2,nfout, freq_out,f_stem,

: namp1,fresp1, numSiteAmp, siteAmp,iseed, nsims,damp,

: Islipweight, slipWeights,iRow,jColumn,pulsingPercent,

: iPapaFlag,PapaGama,PapaNu,PapaT0,PapaPeak,Low_frequencies_taper,

: lowFreqCoeff, psaResidual,ioSim,simpsa,psa,freq,stationNumber,

: earthID) 
end if !Low Residuals

else if(avgResHigh.gt.0.01) then !high Residual above limit if(avgResLow.lt.-0.1) then !low Residual below limit write (ioOutput, '("Case: IB1")')

highStressLimit $=$ stress +100.0

lowStressLimit $=$ stress

if(highStressLimit.gt.999.0) highStresslimit $=999.0$

stress = bestStress(highStressLimit,lowStressLimit,stress,freqC,

: highFreqLimit,error,ioInput,FaultStrike,FaultDip,h,

: numberOfFaults,segFaultLat,

: segFaultLon,segFaultStrike, segFaultDip,segFaultLength,FaultLat,

: FaultLon,siteLat, siteLon,FaultLength, FaultWidth,dl_in, dw_in,

: nl,nw,nsubs,segnl, endOfFaultSegment,y,i0_in,j0_in,nHypocenters,

: dt, beta, rho, amag,iKapa,fmax,Q0,eta, Qmin,

: rg1,rg2,pow1,pow2,pow3,rmin,rd1,rd2,durmin,b1,b2,b3,iwind,

: flocut, nslope,nfreq,freq 1,freq2,nfout, freq_out,f_stem,

: namp1,fresp1, numSiteAmp, siteAmp,iseed, nsims,damp,

: Islipweight, slipWeights,iRow,jColumn,pulsingPercent,

: iPapaFlag,PapaGama,PapaNu,PapaT0,PapaPeak,Low_frequencies_taper,

: lowFreqCoeff, psaResidual,ioSim,simpsa,psa,freq,stationNumber,

: earthID)

else if(avgResLow.gt.0.1) then !Low Residual above limit

write (ioOutput, '('Impossible Situation Occurred Case IB2")')

residualError $=1$

else !Low Residual within limits

write (ioOutput, '('Impossible Situation Occurred Case IB3")')

residualError $=1$

end if !Low Residuals

else !high Residual within limits

if(avgResLow.It.-0.1) then !low Residual below limit

write (ioOutput, '('Case: IC1")')

highLFC $=$ lowFreqCoeff +0.5

lowLFC $=$ lowFreqCoeff -1.0

if $($ lowLFC.lt. -4.0$)$ lowLFC $=-4.0$

if(highLFC.gt.0.75) highLFC $=0.75$

highLFC $=$ lowFreqCoeff

lowLFC $=$ lowFreqCoeff -0.5

if(lowLFC.le.0.01) lowLFC $=0.01$ 
if(highLFC.gt. 2.0) highLFC $=2.0$

lowFreqCoeff $=$ bestLFC (highLFC, lowLFC, lowFreqCoeff,freqC,error, : ioInput,FaultStrike,FaultDip,h,numberOfFaults,segFaultLat, : segFaultLon,segFaultStrike, segFaultDip,segFaultLength,FaultLat, : FaultLon,siteLat, siteLon,FaultLength, FaultWidth,dl_in, dw_in, : nl,nw,nsubs,segnl, endOfFaultSegment,y,i0_in,j0_in,nHypocenters, : dt, beta, rho, amag,stress,iKapa,fmax,Q0,eta, Qmin,

: rg1,rg2,pow1, pow2,pow3,rmin,rd1,rd2,durmin,b1,b2,b3, iwind, : flocut, nslope,nfreq,freq 1,freq2,nfout, freq_out,f_stem, : namp1,fresp1, numSiteAmp, siteAmp,iseed, nsims,damp, : Islipweight, slipWeights,iRow,jColumn,pulsingPercent, : iPapaFlag,PapaGama,PapaNu,PapaT0,PapaPeak,Low_frequencies_taper, : psaResidual,ioSim,simpsa,psa,freq,stationNumber,earthID)

else if(avgResLow.gt.0.1) then !Low Residual above limit write (ioOutput, '('Impossible Situation Occurred Case IC2")') residualError $=1$

else !Low Residual within limits write (ioOutput, '('Impossible Situation Occurred Case IC3")') residualError $=1$ end if !Low Residuals end if !high Residuals

else if(totalAvgRes.gt.0.05) then !totalAvgRes above limit if(avgResHigh.lt.-0.01) then !high Residual below limit if(avgResLow.It.-0.1) then !low Residual below limit write (ioOutput, '('Impossible Situation Occurred Case IIA1")') residualError $=1$ else if(avgResLow.gt.0.1) then !Low Residual above limit write (ioOutput, '('Case: IIA2")') lowLFC $=$ lowFreqCoeff -0.25 highLFC $=$ lowFreqCoeff +0.5 if(lowLFC.1t. -4.0$)$ lowLFC $=-4.0$ if(highLFC.gt.0.75) highLFC $=0.75$ highLFC $=$ lowFreqCoeff +0.25 lowLFC $=$ lowFreqCoeff -0.1 if(lowLFC.le.0.01) lowLFC $=0.01$ if(highLFC.gt.2.0) highLFC $=2.0$

lowFreqCoeff = bestLFC(highLFC, lowLFC,lowFreqCoeff, freqC,error, : ioInput,FaultStrike,FaultDip,h,numberOfFaults,segFaultLat, 
: segFaultLon,segFaultStrike, segFaultDip,segFaultLength,FaultLat,

: FaultLon,siteLat, siteLon,FaultLength, FaultWidth,dl_in, dw_in,

: nl,nw,nsubs,segnl, endOfFaultSegment,y,i0_in,j0_in,nHypocenters,

: dt, beta, rho, amag,stress, iKapa,fmax,Q0,eta, Qmin,

: rg1,rg2,pow1,pow2,pow3,rmin,rd1,rd2,durmin,b1,b2,b3,iwind,

: flocut, nslope,nfreq,freq1,freq2,nfout, freq_out,f_stem,

: namp1,fresp1, numSiteAmp, siteAmp,iseed, nsims,damp,

: Islipweight, slipWeights,iRow,jColumn,pulsingPercent,

: iPapaFlag,PapaGama,PapaNu,PapaT0,PapaPeak,Low_frequencies_taper,

: psaResidual,ioSim,simpsa,psa,freq,stationNumber,earthID)

else !Low Residual within limits

write (ioOutput, '('Impossible Situation Occurred Case IIA3")')

residualError $=1$

end if !Low Residuals

else if(avgResHigh.gt.0.01) then !high Residual above limit

if(avgResLow.lt.-0.1) then !low Residual below limit

write (ioOutput, '('Case: IIB1")')

highStressLimit $=$ stress +100.0

lowStressLimit $=$ stress

if(highStressLimit.gt.999.0) highStresslimit $=999.0$

stress $=$ bestStress(highStressLimit,lowStressLimit,stress,freqC,

: highFreqLimit,error,ioInput,FaultStrike,FaultDip,h,

: numberOfFaults,segFaultLat,

: segFaultLon,segFaultStrike, segFaultDip,segFaultLength,FaultLat,

: FaultLon,siteLat, siteLon,FaultLength, FaultWidth,dl_in, dw_in,

: nl,nw,nsubs,segnl, endOfFaultSegment,y,i0_in,j0_in,nHypocenters,

: dt, beta, rho, amag,iKapa,fmax,Q0,eta, Qmin,

: rg1,rg2,pow1,pow2,pow3,rmin,rd1,rd2,durmin,b1,b2,b3,iwind,

: flocut, nslope,nfreq,freq 1 ,freq2,nfout, freq_out,f_stem,

: namp1,fresp1, numSiteAmp, siteAmp,iseed, nsims,damp,

: Islipweight, slipWeights,iRow,jColumn,pulsingPercent,

: iPapaFlag,PapaGama,PapaNu,PapaT0,PapaPeak,Low frequencies_taper,

: lowFreqCoeff, psaResidual,ioSim,simpsa,psa,freq,stationNumber,

: earthID)

else if(avgResLow.gt.0.1) then !Low Residual above limit

write (ioOutput, '('Case: IIB2")')

highStressLimit $=$ stress +100.0

lowStressLimit $=$ stress 
if(highStressLimit.gt.999.0) highStresslimit $=999.0$

stress = bestStress(highStressLimit,lowStressLimit,stress,freqC,

: highFreqLimit,error,ioInput,FaultStrike,FaultDip,h,

: numberOfFaults,segFaultLat,

: segFaultLon,segFaultStrike, segFaultDip,segFaultLength,FaultLat,

: FaultLon,siteLat, siteLon,FaultLength, FaultWidth,dl_in, dw_in,

: nl,nw,nsubs,segnl, endOfFaultSegment,y,i0_in,j0_in,nHypocenters,

: dt, beta, rho, amag,iKapa,fmax,Q0,eta, Qmin,

: rg1,rg2,pow1,pow2,pow3,rmin,rd1,rd2,durmin,b1,b2,b3,iwind,

: flocut, nslope,nfreq,freq 1 ,freq2,nfout, freq_out,f_stem,

: namp1, fresp1, numSiteAmp, siteAmp,iseed, nsims,damp,

: Islipweight, slipWeights,iRow,jColumn,pulsingPercent,

: iPapaFlag,PapaGama,PapaNu,PapaT0,PapaPeak,Low_frequencies_taper,

: lowFreqCoeff, psaResidual,ioSim,simpsa,psa,freq,stationNumber,

: earthID)

else !Low Residual within limits

write (ioOutput, '('Case: IIB3")')

highStressLimit $=$ stress +100.0

lowStressLimit $=$ stress

if(highStressLimit.gt.999.0) highStresslimit $=999.0$

stress $=$ bestStress $($ highStressLimit,lowStressLimit,stress,freqC,

: highFreqLimit,error,ioInput,FaultStrike,FaultDip,h,

: numberOfFaults,segFaultLat,

: segFaultLon,segFaultStrike, segFaultDip,segFaultLength,FaultLat,

: FaultLon,siteLat, siteLon,FaultLength, FaultWidth,dl_in, dw_in,

: nl,nw,nsubs,segnl, endOfFaultSegment,y,i0_in,j0_in,nHypocenters,

: dt, beta, rho, amag,iKapa,fmax,Q0,eta, Qmin,

: rg1,rg2,pow1,pow2,pow3,rmin,rd1,rd2,durmin,b1,b2,b3,iwind,

: flocut, nslope,nfreq,freq1,freq2,nfout, freq_out,f_stem,

: namp1,fresp1, numSiteAmp, siteAmp,iseed, nsims,damp,

: Islipweight, slipWeights,iRow,jColumn,pulsingPercent,

: iPapaFlag,PapaGama,PapaNu,PapaT0,PapaPeak,Low_frequencies_taper,

: lowFreqCoeff, psaResidual,ioSim,simpsa,psa,freq,stationNumber,

: earthID)

end if !Low Residuals

else !high Residual within limits

if(avgResLow.lt.-0.1) then !low Residual below limit

write (ioOutput, '('Impossible Situation Occurred Case IIC1")')

residualError $=1$ 
else if(avgResLow.gt.0.1) then !Low Residual above limit write (ioOutput, '('Case: IIC2")')

highLFC $=$ lowFreqCoeff +0.25

lowLFC $=$ lowFreqCoeff -0.5

if(lowLFC.lt. -4.0$)$ lowLFC $=-4.0$

if(highLFC.gt.0.75) highLFC $=0.75$

highLFC $=$ lowFreqCoeff +0.25

lowLFC $=$ lowFreqCoeff -0.1

if(lowLFC.le.0.01) lowLFC $=0.01$

if(highLFC.gt.2.0) highLFC $=2.0$

lowFreqCoeff $=$ bestLFC $($ highLFC, lowLFC,lowFreqCoeff,freqC,error,

: ioInput,FaultStrike,FaultDip,h,numberOfFaults,segFaultLat,

: segFaultLon,segFaultStrike, segFaultDip,segFaultLength,FaultLat,

: FaultLon,siteLat, siteLon,FaultLength, FaultWidth,dl_in, dw_in,

: nl,nw,nsubs,segnl, endOfFaultSegment,y,i0_in,j0_in,nHypocenters,

: dt, beta, rho, amag,stress,iKapa,fmax,Q0,eta, Qmin,

: rg1,rg2,pow1,pow2,pow3,rmin,rd1,rd2,durmin,b1,b2,b3,iwind,

: flocut, nslope,nfreq,freq 1,freq2,nfout, freq_out,f_stem,

: namp1,fresp1, numSiteAmp, siteAmp,iseed, nsims,damp,

: Islipweight, slipWeights,iRow,jColumn,pulsingPercent,

: iPapaFlag,PapaGama,PapaNu,PapaT0,PapaPeak,Low_frequencies_taper,

: psaResidual,ioSim,simpsa,psa,freq,stationNumber,earthID)

else !Low Residual within limits

write (ioOutput, '('Impossible Situation Occurred Case IIC3")')

residualError $=1$

end if !Low Residuals

end if !high Residuals

else !totalAvgRes within limits

if(avgResHigh.It.-0.01) then !high Residual below limit

if(avgResLow.lt.-0.1) then !low Residual below limit

write (ioOutput, '('Impossible Situation Occurred Case IIIA1")')

residualError $=1$

else if(avgResLow.gt.0.1) then !Low Residual above limit

write (ioOutput, '("Case: IIIA2")')

highStressLimit $=$ stress

lowStressLimit $=$ stress -100.0

if(lowStressLimit.lt.1) lowStresslimit $=1.0$

stress $=$ bestStress $($ highStressLimit,lowStressLimit,stress, freqC, 
: highFreqLimit,error,ioInput,FaultStrike,FaultDip,h,

: numberOfFaults, segFaultLat,

: segFaultLon,segFaultStrike, segFaultDip,segFaultLength,FaultLat,

: FaultLon,siteLat, siteLon,FaultLength, FaultWidth,dl_in, dw_in,

: nl,nw,nsubs,segnl, endOfFaultSegment,y,i0_in,j0_in,nHypocenters,

: dt, beta, rho, amag,iKapa,fmax,Q0,eta, Qmin,

: rg1,rg2,pow1,pow2,pow3,rmin,rd1,rd2,durmin,b1,b2,b3,iwind,

: flocut, nslope,nfreq,freq1,freq2,nfout, freq_out,f_stem,

: namp1,fresp1, numSiteAmp, siteAmp,iseed, nsims,damp,

: Islipweight, slipWeights,iRow,jColumn,pulsingPercent,

: iPapaFlag,PapaGama,PapaNu,PapaT0,PapaPeak,Low_frequencies_taper,

: lowFreqCoeff, psaResidual,ioSim,simpsa,psa,freq,stationNumber,

: earthID)

else !Low Residual within limits

write (ioOutput, '("Case IIIA3")')

highStressLimit $=$ stress

lowStressLimit $=$ stress -100.0

if (lowStressLimit.lt.1) lowStresslimit $=1.0$

stress $=$ bestStress(highStressLimit,lowStressLimit,stress,freqC,

: highFreqLimit,error,ioInput,FaultStrike,FaultDip,h,

: numberOfFaults, segFaultLat,

: segFaultLon,segFaultStrike, segFaultDip,segFaultLength,FaultLat,

: FaultLon,siteLat, siteLon,FaultLength, FaultWidth,dl_in, dw_in, : nl,nw,nsubs,segnl, endOfFaultSegment,y,i0_in,j0_in,nHypocenters, : dt, beta, rho, amag,iKapa,fmax,Q0,eta, Qmin,

: rg1,rg2,pow1,pow2,pow3,rmin,rd1,rd2,durmin,b1,b2,b3, iwind,

: flocut, nslope,nfreq,freq 1 ,freq2,nfout, freq_out,f_stem,

: namp1,fresp1, numSiteAmp, siteAmp,iseed, nsims,damp,

: Islipweight, slipWeights,iRow,jColumn,pulsingPercent,

: iPapaFlag,PapaGama,PapaNu,PapaT0,PapaPeak,Low_frequencies_taper,

: lowFreqCoeff, psaResidual,ioSim,simpsa,psa,freq,stationNumber,

: earthID)

end if !Low Residuals

else if(avgResHigh.gt.0.01) then !high Residual above limit

if(avgResLow.lt.-0.1) then !low Residual below limit

write (ioOutput, '('Case: IIIB1")')

highStressLimit $=$ stress +100.0

lowStressLimit $=$ stress

if(highStressLimit.gt.999.0) highStresslimit $=999.0$ 
stress = bestStress(highStressLimit,lowStressLimit,stress,freqC, : highFreqLimit,error,ioInput,FaultStrike,FaultDip,h,

: numberOfFaults,segFaultLat,

: segFaultLon,segFaultStrike, segFaultDip,segFaultLength,FaultLat,

: FaultLon,siteLat, siteLon,FaultLength, FaultWidth,dl_in, dw_in,

: nl,nw,nsubs,segnl, endOfFaultSegment,y,i0_in,j0_in,nHypocenters,

: dt, beta, rho, amag,iKapa,fmax,Q0,eta, Qmin,

: rg1,rg2,pow1,pow2,pow3,rmin,rd1,rd2,durmin,b1,b2,b3,iwind,

: flocut, nslope,nfreq,freq1,freq2,nfout, freq_out,f_stem,

: namp1,fresp1, numSiteAmp, siteAmp,iseed, nsims,damp,

: Islipweight, slipWeights,iRow,jColumn,pulsingPercent,

: iPapaFlag,PapaGama,PapaNu,PapaT0,PapaPeak,Low frequencies_taper,

: lowFreqCoeff, psaResidual,ioSim,simpsa,psa,freq,stationNumber,

: earthID)

else if(avgResLow.gt.0.1) then !Low Residual above limit write (ioOutput, '('Impossible Situation Occurred Case IIIB2")')

residualError $=1$

else !Low Residual within limits

write (ioOutput, '("Case IIIB3")')

highStressLimit $=$ stress +100.0

lowStressLimit $=$ stress

if(highStressLimit.gt.999.0) highStresslimit $=999.0$

stress $=$ bestStress(highStressLimit,lowStressLimit,stress,freqC,

: highFreqLimit,error,ioInput,FaultStrike,FaultDip,h,

: numberOfFaults,segFaultLat,

: segFaultLon,segFaultStrike, segFaultDip,segFaultLength,FaultLat,

: FaultLon,siteLat, siteLon,FaultLength, FaultWidth,dl_in, dw_in,

: nl,nw,nsubs,segnl, endOfFaultSegment,y,i0_in,j0_in,nHypocenters,

: dt, beta, rho, amag,iKapa,fmax,Q0,eta, Qmin,

: rg1,rg2,pow1,pow2,pow3,rmin,rd1,rd2,durmin,b1,b2,b3,iwind,

: flocut, nslope,nfreq,freq 1 ,freq 2 ,nfout, freq_out,f_stem,

: namp1,fresp1, numSiteAmp, siteAmp,iseed, nsims,damp,

: Islipweight, slipWeights,iRow,jColumn,pulsingPercent,

: iPapaFlag,PapaGama,PapaNu,PapaT0,PapaPeak,Low_frequencies_taper,

: lowFreqCoeff, psaResidual,ioSim,simpsa,psa,freq,stationNumber,

: earthID)

end if !Low Residuals

else !high Residual within limits 
if(avgResLow.lt.-0.1) then !low Residual below limit write (ioOutput, '('Case: IIIC1')')

highLFC $=$ lowFreqCoeff +0.25

lowLFC $=$ lowFreqCoeff -0.5

if(lowLFC.1t. -4.0$)$ lowLFC $=-4.0$

if(highLFC.gt.0.75) highLFC $=0.75$

highLFC $=$ lowFreqCoeff

lowLFC $=$ lowFreqCoeff -0.5

if(lowLFC.1t.0.01) lowLFC $=0.01$

if(highLFC.gt.2.0) highLFC $=2.0$

lowFreqCoeff $=$ bestLFC (highLFC, lowLFC,lowFreqCoeff, freqC,error,

: ioInput,FaultStrike,FaultDip,h,numberOfFaults,segFaultLat,

: segFaultLon,segFaultStrike, segFaultDip,segFaultLength,FaultLat,

: FaultLon,siteLat, siteLon,FaultLength, FaultWidth,dl_in, dw_in,

: nl,nw,nsubs,segnl, endOfFaultSegment,y,i0_in,j0_in,nHypocenters,

: dt, beta, rho, amag,stress,iKapa,fmax,Q0,eta, Qmin,

: rg1,rg2,pow1,pow2,pow3,rmin,rd1,rd2,durmin,b1,b2,b3,iwind,

: flocut, nslope,nfreq,freq1,freq2,nfout, freq_out,f_stem,

: namp1,fresp1, numSiteAmp, siteAmp,iseed, nsims,damp,

: Islipweight, slipWeights,iRow,jColumn,pulsingPercent,

: iPapaFlag,PapaGama,PapaNu,PapaT0,PapaPeak,Low_frequencies_taper,

: psaResidual,ioSim,simpsa,psa,freq,stationNumber,earthID)

else if(avgResLow.gt.0.1) then !Low Residual above limit

write (ioOutput, '('Case: IIIC2")')

lowLFC $=$ lowFreqCoeff -0.25

highLFC $=$ lowFreqCoeff +0.5

if(highLFC.gt.0.75) highLFC $=0.75$

if (lowLFC.It. -4.0$)$ lowLFC $=-4.0$

highLFC $=$ lowFreqCoeff +0.25

lowLFC $=$ lowFreqCoeff -0.1

if(lowLFC.le.0.01) lowLFC $=0.01$

if(highLFC.gt.2.0) highLFC $=2.0$

lowFreqCoeff $=$ bestLFC (highLFC, lowLFC,lowFreqCoeff,freqC,error, : ioInput,FaultStrike,FaultDip,h,numberOfFaults,segFaultLat,

: segFaultLon,segFaultStrike, segFaultDip,segFaultLength,FaultLat,

: FaultLon,siteLat, siteLon,FaultLength, FaultWidth,dl_in, dw_in,

: nl,nw,nsubs,segnl, endOfFaultSegment,y,i0_in,j0_in,nHypocenters,

: dt, beta, rho, amag,stress,iKapa,fmax,Q0,eta, Qmin,

: rg1,rg2,pow1,pow2,pow3,rmin,rd1,rd2,durmin,b1,b2,b3,iwind, 
: flocut, nslope,nfreq,freq1,freq2,nfout, freq_out,f_stem, : namp1,fresp1, numSiteAmp, siteAmp,iseed, nsims,damp, : Islipweight, slipWeights,iRow,jColumn,pulsingPercent, : iPapaFlag,PapaGama,PapaNu,PapaT0,PapaPeak,Low_frequencies_taper, : psaResidual,ioSim,simpsa,psa,freq,stationNumber,earthID)

else !Low Residual within limits write (ioOutput, '('Case: IIIC3")') resNotGood $=$.FALSE. end if !Low Residuals end if !high Residuals end if !total Residuals

doWhileCount $=$ doWhileCOunt +1.0

if(doWhileCount.ge.20.0) then write(ioOutput, '('Too many times through loop")') residualError $=-1.0$ end if

if(tempStress.eq.stress.and.tempLFC.eq.lowFreqCoeff) then write(ioOutput, '("No change from last time through")') residualError $=-1.0$

end if

end do !do while loop for residuals

if (residualError.le.0.0) then

write (ioOutput, '('Stress: ",f5.1)') stress

write (ioOutput, '('Low Frequency Coefficient: ",f7.4)')

: lowFreqCoeff

write (ioOutput, '('Site Lat: ",f6.3)') SiteLat

write (ioOutput, '('Site Lon: ",f8.3)') SiteLon

write (ioOutput, '('Freq PSA SimulatedPSA Residual')')

write (ioOutput,'((f7.3,1x,e 11.4,1x,e11.4,1x,f10.4))')

: (freq(i), psa(i), simpsa(i), psaRes(i), i=1,105)

end if

error $=0$ 
close (ioOutput)

stationNumber $=$ stationNumber +1

end if !(morestation and NEHRP)

end do !more stations loop

close (ioStation)

Standard Fortran 90 intrinsic Subroutine DATE_AND_TIME

call DATE_AND_TIME( datx, time_stop )

Date is returned as 'CCYYMMDD'

Time is returned as 'hhmmss.sss'

call time_diff(time_start, time_stop, time_elapsed)

print *,

:' Elapsed time (sec): ', time_elapsed

call system('pause')

stop

end

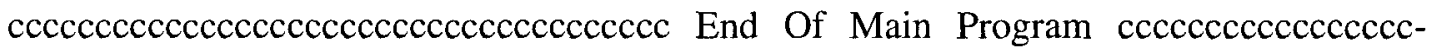
$\operatorname{coc} \operatorname{ccc} \operatorname{ccccccc}$

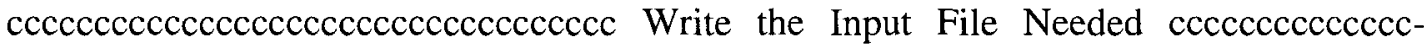
$\operatorname{cccccc}$

subroutine writeInputFile(ioInput,

: FaultStrike,FaultDip,h,

: numberOfFaults,

: segFaultLat, segFaultLon,

: segFaultStrike, segFaultDip,

: segFaultLength,

: FaultLat,FaultLon,

: siteLat, siteLon,

: FaultLength, FaultWidth,

: dl_in, dw_in, nl, nw, nsubs,

: segnl, endOfFaultSegment,

$: y$,

: i0_in,j0_in,nHypocenters,

: dt, beta, rho, amag,

: stress,iKapa,fmax,

: Q0,eta, Qmin, 
: rg1,rg2,pow1,pow2,pow3,

: rmin,rd1,rd2,durmin,b1,b2,b3, ! trilinear duration parameters

: iwind,

: flocut, nslope,

: nfreq,freq1,freq2,

: nfout, freq_out,

: f_stem,

: numAmpFile, ampFile, numSiteAmp, siteAmp,

: iseed, nsims,damp,

: Islipweight, slipWeights,

: iRow,jColumn,pulsingPercent,

: iPapaFlag,PapaGama,PapaNu,PapaT0,PapaPeak,

: Low_frequencies_taper,

: D_Motazedian_Taper_coef)

real freq_out $(*)$

real dl_in, $d w \_$in

integer segmentNL, numAmpFile, numSiteAmp

dimension slipWeights $(200,200)$, siteLocation $(300,2)$

dimension segFaultLat(10,2),segFaultLon(10,2),segFaultStrike(10),

: segFaultDip(10),segFaultLength(10)

dimension $\mathrm{dl}(10)$, segnl(10)

integer endOfFaultSegment(10)

character f_stem*(*),

: ampFile*30, siteAmp*30,aline*60,

: version_ctl*8, version_in*30

character cmnts 2 skip $(50) * 80$, buf_in $* 10$

write (ioInput, '("!Control file for program exsim_beta")')

write (ioInput, '('!Revision of program involving a change in the :control file on this date:")')

write (ioInput, '(" 02/10/10")')

write (ioInput, '("!Title")')

write (ioInput, '('Runs for low frequency calibration")')

write (ioInput, '("!MW, Stress, flag ( $0=$ fmax; 1=kappa),

:fmax or kappa")')

write (ioInput,'(f6.3,1x,f5.1,1x,i1,1x,f8.5)') amag,stress,iKapa, :fmax 
write (ioInput, '("!number of Fault segments, width of fault, and :depth of fault (to upper edge)"')')

write (ioInput, '(i2,1x,f5.2,1x,f5.2)' ) numberOfFaults, :FaultWidth, h

write (ioInput, '("!lat and lon of upper edge of fault segment :beginning and end and dip of fault segment")') write (ioInput, '("!must have as many lines as number of fault :segments above')') do $\mathrm{i}=1$, numberOfFaults write (ioInput, '(f10.5,1x,f10.5,1x,f10.5,1x,f10.5,1x,f5.2)')

: segFaultLat(i,1),segFaultLon(i,1),segFaultLat(i,2),

: segFaultLon(i,2),segFaultDip(i)

end do

write (ioInput, '("!subfault length and width")')

write (ioInput, '(f5.2,1x,f5.2)') dl_in, dw_in

write (ioInput, '('!hypo location in $(\mathrm{i}, \mathrm{j})$ sub. $\mathrm{i}=0$ or $\mathrm{j}=0$ for a :random hypo. number of hypocenters to iterate over")') write (ioInput, '(i3,1x,i3,1x,i2)') i0_in,j0_in, nHypocenters

write (ioInput, '("'beta , rho, y (=vrup/beta),delta t")')

write (ioInput, '(f4.2,1x,f4.2,1x,f3.2,1x,f5.3)')

:beta,rho, $y, d t$

write (ioInput, '('!gsprd: rg1,rg2,pow1,pow2,pow3")')

write (ioInput, '(f4.1,1x,f5.1,1x,f4.1,1x,f4.1,1x,f4.1)')

:rg1,rg2,pow1,pow2,pow3

write (ioInput, '("!Q: Q0,eta, Qmin")')

write (ioInput, '(f5.1,1x,f5.3,1x,f6.1)') Q0,eta, Qmin

write (ioInput, '("!trilinear duration and properties

:(rmin,rd1,rd2,durmin,b1,b2,b3)"')')

write (ioInput, '(f4.1,1x,f5.1,1x,f5.1,1x,f5.2,1x,f4.2,1x,f4.2,1x, :f4.2)’)rmin,rd1,rd2,durmin,b1,b2,b3

write (ioInput, '("!Type of window: 1 for Saragoni-Hart :taper windows, 0 for tapered boxcar window")')

write (iolnput, '(i1)') iwind

write (ioInput, '("!low-cut filter comer(0- i no filter), 
:nslope")')

write (ioInput, '(f5.3,1x,i2)') flocut, nslope

write (ioInput, '("!

write (ioInput, '(f5.2)') damp

write (ioInput, '("!\# of $\mathrm{f}$ and Min and Max F for response spectra :(Max F should be less than Nyquist F from delta t)')')

write (ioInput, '(i3,1x,f4.1,1x,f5.1)') nfreq,freq1, freq2

write (ioInput, '("!no. of frequencies for summary output

:(10 max $): ") ')$

write (ioInput, '(i3)') nfout

write (ioInput, '("'frequencies in summary output (-1.0 for pgv

:and 99.0 for pga):")')

write (ioInput, '(f5.2,1x)') (freq_out(i),i=1,nfout)

write (ioInput, '("!Output file names stem:")')

write (ioInput, '(a)') f_stem

write (ioInput, '("!Name of crustal amplification file:")')

write (ioInput, '(a)') ampFile

write (ioInput, '("!No. of frequencies in the crustal

:amplification file")')

write (ioInput, '(i2)') numAmpFile

write (ioInput, '("'Name of site amplification file:")')

write (ioInput, '(a)') siteAmp

write (ioInput, '("'No. of frequencies in the site amplification

:file (0 to skip)")')

write (ioInput, '(i2)') numSiteAmp

write (ioInput, '("'Low frequencies taper( $0=$ Boores taper,

$: 1=$ Motazedians taper), low frequency Coef for Motazedians taper :(needs and entry)")')

write (ioInput, '(i1,1x,f7.4)')Low_frequencies_taper,

:D_Motazedian_Taper_coef

write (ioInput, '("'Pulsing Percent")')

write (ioInput, '(f5.1)')pulsingPercent 
write (ioInput, '('!deterministic flag(use 0!),gama,nu,t0, impulse : peak")')

write (ioInput, '(i1,1x,f3.1,1x,f5.1,1x,f3.1,1x,f4.1)')iPapaFlag, :PapaGama,PapaNu,PapaT0,PapaPeak

write (ioInput, '("!iseed, \# of trials")')

write (ioInput, '(i5,1x,i4)') iseed, nsims

write (ioInput, '("'Number of Sites, coord flag (1=lat,long;

:2=R,Az; 3=N,E)")')

write (ioInput, '(i2,1x,i1)')1, 1

write (ioInput, '("!Coordinates of each site")')

write (ioInput, '(f10.5,1x,f10.5)') siteLat,siteLon

write (ioInput, '("! islipweight $=-1-i$ unity slip for all subfaults

$:=0-i$ specify slips below, $=1-i$ random weights")')

write (ioInput, '(i2)') IslipWeight

write (ioInput, '("!Matrix of slip weights")')

write (ioInput, '(f3.1,1x)') ((slipWeights $(\mathrm{i}, \mathrm{j}), \mathrm{i}=1, \mathrm{nl}), \mathrm{j}=1, \mathrm{nw})$

return

end

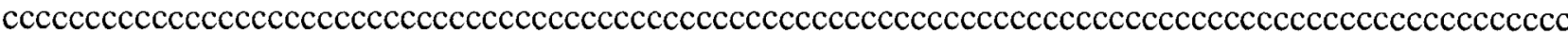

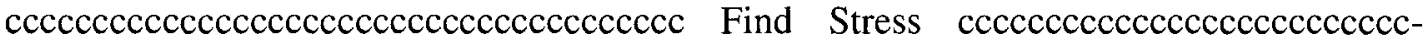

$\operatorname{cccccc}$

function bestStress(highStressLimit, lowStressLimit,

: lowFreqLimit, highFreqLimit, error,

: ioInput,FaultStrike,FaultDip,h,numberOfFaults,segFaultLat,

: segFaultLon,segFaultStrike, segFaultDip,segFaultLength,FaultLat,

: FaultLon,siteLat, siteLon,FaultLength, FaultWidth,dl_in, dw_in,

: nl,nw,nsubs,segnl, endOfFaultSegment,y,i0_in,j0_in,nHypocenters,

: dt, beta, rho, amag,iKapa,fmax,Q0,eta, Qmin,

: rg1,rg2,pow1,pow2,pow3,rmin,rd1,rd2,durmin,b1,b2,b3,iwind,

: flocut, nslope,nfreq,freq 1 ,freq2,nfout, freq_out,f_stem,

: namp1,fresp1, numSiteAmp, siteAmp,iseed, nsims,damp,

: Islipweight, slipWeights,iRow,jColumn,pulsingPercent,

: iPapaFlag,PapaGama,PapaNu,PapaT0,PapaPeak,Low_frequencies_taper, 
: lowFreqCoeff, psaResidual, ioSim,simpsa,psa,freq,stationNumber, : earthID)

character InputFileName*30,f_stem $* 30$,fresp $1 * 30$,fresp $2 * 30$

character ampFile*30, siteAmp*30, flfc*30

dimension slipWeights $(200,200)$, siteLocation $(300,2)$

dimension segFaultLat(10,2), segFaultLon(10,2), segFaultStrike(10), : segFaultDip(10),segFaultLength(10),psa(105),freq(105)

dimension dl(10), segnl(10), psaResidual(105,3), simpsa(105)

real highStress, lowStress, midStress, lowFreqLimit, highFreqLimit

real avgResLow, avgResHigh, avgResMid, StressDiff

integer error, freqCount, endOfFaultSegment(10)

real lowFreqCoeff, highStressLimit, lowStressLimit

real freq_out $(*)$

integer stationNumber, earthID, numAmpFile, numSiteAmp

highStress $=$ highStressLimit

lowStress $=$ lowStressLimit

error $=0$

c Create the name for the output files

f_stem = 'Output_000_000_00000_0000000'

write(f_stem(8:10),'(i3.3)') earthID

write(f_stem(12:14),'(i3.3)')stationNumber

write(f_stem(16:20),'(f5.1)') lowStress

write(f_stem(22:28),'(f7.4)') lowFreqCoeff

call trim_c(f_stem, nc_f_stem)

open (iolnput, file='EXSIM_MFIn.in',status='unknown')

call writeInputFile(ioInput,

: FaultStrike,FaultDip,h,

: numberOfFaults,

: segFaultLat, segFaultLon,

: segFaultStrike, segFaultDip,

: segFaultLength,

: FaultLat,FaultLon,

: siteLat, siteLon,

: FaultLength, FaultWidth,

: dl_in, dw_in, nl, nw, nsubs,

: segnl, endOfFaultSegment,

: $y$,

: i0_in,j0_in,nHypocenters, 
: dt, beta, rho, amag,

: lowStress,iKapa,fmax,

: Q0,eta, Qmin,

: rg1,rg2,pow1,pow2,pow3,

: rmin,rd1,rd2,durmin,b1,b2,b3, ! trilinear duration parameters

: iwind,

: flocut, nslope,

: nfreq,freq1,freq2,

: nfout, freq_out,

: f_stem,

: namp1,fresp1, numSiteAmp, siteAmp,

: iseed, nsims,damp,

: Islipweight, slipWeights,

: iRow,jColumn,pulsingPercent,

: iPapaFlag,PapaGama,PapaNu,PapaT0,PapaPeak,

: Low_frequencies_taper,

: lowFreqCoeff)

close(ioInput)

call system('EXSIM_Steve2.exe')

open(ioSim, file='simulatedParameters.out', status='unknown')

read $\left(\mathrm{ioSim},{ }^{*}\right)(\operatorname{simpsa}(\mathrm{j}), \mathrm{j}=1,105)$

close(ioSim)

do $\mathrm{i}=1,105$

$\operatorname{simpsa}(\mathrm{i})=\operatorname{simpsa}(\mathrm{i}) / 981.0$

end do

do $i=1,105$

psaResidual $(\mathrm{i}, 1)=\log (\mathrm{psa}(\mathrm{i}))-\log (\operatorname{simpsa}(\mathrm{i}))$

end do

avgResLow $=0$

freqCount $=0$

do $i=1,105$

if (freq(i).ge.lowFreqLimit.and.freq(i).le.highFreqLimit) then

avgResLow $=\operatorname{avgResLow~}+$ psaResidual $(i, 1)$

freqCount $=$ freqCount +1

end if

end do

avgResLow $=$ avgResLow/float(freqCount) 
if (avgResLow.le.0) then

print *, 'stress needed is below low Stress limit for

:station number:',stationNumber

error $=1$

bestStress $=$ lowStress

end if

if (error.eq.0) then

$c$ Create the name for the output files

f_stem = 'Output_000_000_00000_0000000'

write(f_stem( $8: 10)$,'(i3.3)') earthID

write(f_stem(12:14),'(i3.3)')stationNumber

write(f_stem(16:20),'(f5.1)') highStress

write(f_stem(22:28),'(f7.4)') lowFreqCoeff

call trim_c(f_stem, nc_f_stem)

open (ioInput, file='EXSIM_MFIn.in',status='unknown')

call writeInputFile(ioInput,

: FaultStrike,FaultDip,h,

: numberOfFaults,

: segFaultLat, segFaultLon,

: segFaultStrike, segFaultDip,

: segFaultLength,

: FaultLat,FaultLon,

: siteLat, siteLon,

: FaultLength, FaultWidth,

: dl_in, dw_in, nl, nw, nsubs,

: segnl, endOfFaultSegment,

: $y$,

: i0_in,j0_in, nHypocenters,

: dt, beta, rho, amag,

: highStress,iKapa,fmax,

: Q0,eta, Qmin,

: rg1,rg2,pow1,pow2,pow3,

: rmin,rd1,rd2,durmin,b1,b2,b3, ! trilinear duration parameters

: iwind,

: flocut, nslope,

: nfreq,freq1,freq2,

: nfout, freq_out,

: f_stem,

: namp1,fresp1, numSiteAmp, siteAmp,

: iseed, nsims,damp, 


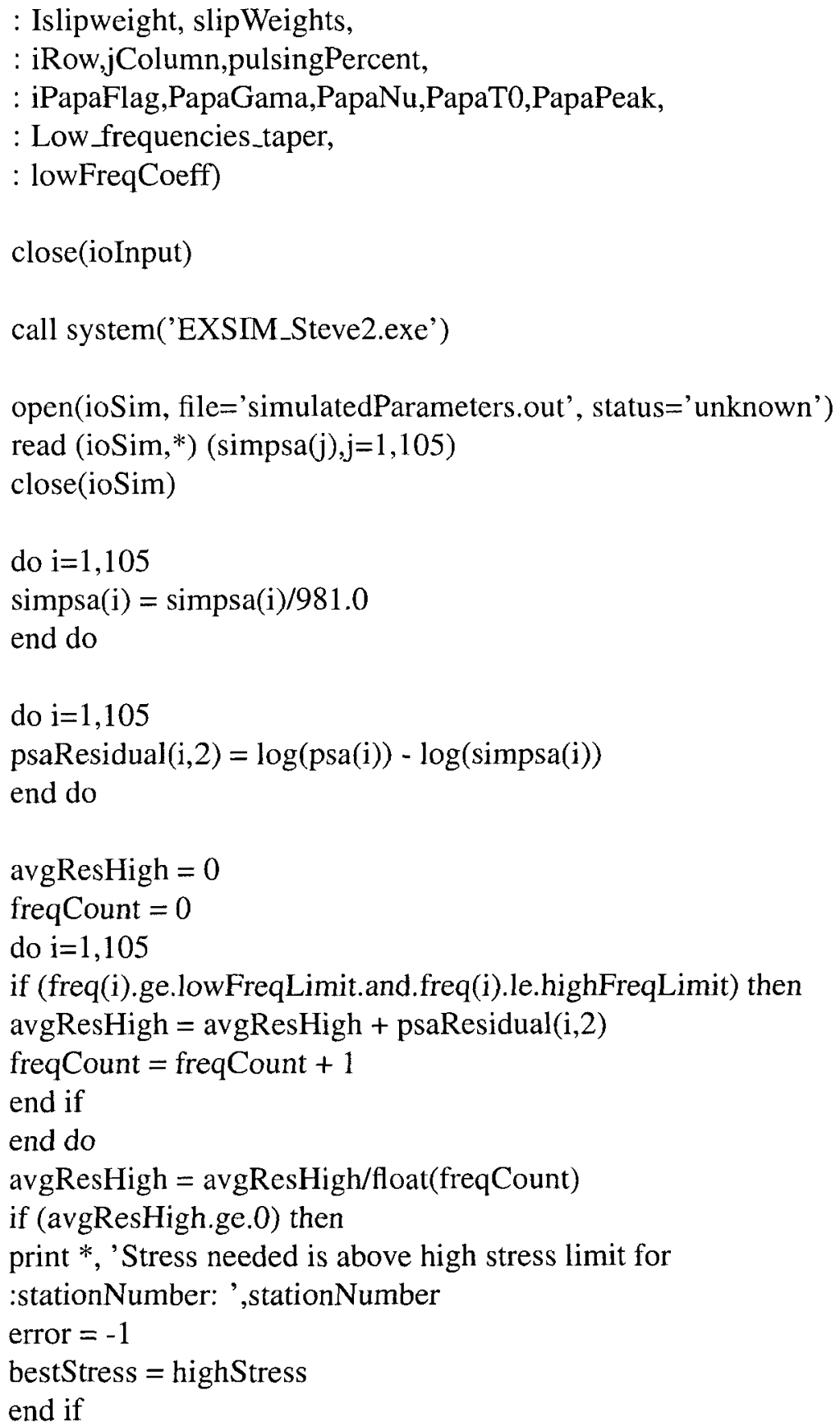

end if !skip this if stress needed is below 1 bar c Now use the bisection method to find the stress drop (limit Residual within 0.001) avgResMid $=1$ stressDiff $=1$ pastStress $=1$ 
if (error.ne.0) avgResMid $=0.0$

do while ((avgResMid.lt.-0.001.or.avgResMid.gt.0.001).and.(

:stressDiff.lt.-0.1.or.stressDiff.gt.0.1))

midStress $=($ highStress + lowStress $) / 2.0$

$c$ Create the name for the output files

f_stem = 'Output_000_000_00000_0000000'

write(f_stem (8:10),'(i3.3)') earthID

write(f_stem(12:14),'(i3.3)')stationNumber

write(f_stem(16:20),'(f5.1)') midStress

write(f_stem(22:28),'(f7.4)') lowFreqCoeff

call trim_c(f_stem, nc_f_stem)

open (ioInput, file='EXSIM_MFIn.in',status='unknown')

call writeInputFile(ioInput,

: FaultStrike,FaultDip,h,

: numberOfFaults,

: segFaultLat, segFaultLon,

: segFaultStrike, segFaultDip,

: segFaultLength,

: FaultLat,FaultLon,

: siteLat, siteLon,

: FaultLength, FaultWidth,

: dl_in, dw_in, nl, nw, nsubs,

: segnl, endOfFaultSegment,

$: y$,

: i0_in,j0_in,nHypocenters,

: dt, beta, rho, amag,

: midStress,iKapa,fmax,

: Q0,eta, Qmin,

: rg1,rg2,pow1,pow2,pow3,

: rmin,rd1,rd2,durmin,b1,b2,b3, ! trilinear duration parameters

: iwind,

: flocut, nslope,

: nfreq,freq1,freq2,

: nfout, freq_out,

: f_stem,

: namp1,fresp1, numSiteAmp, siteAmp,

: iseed, nsims,damp,

: Islipweight, slipWeights,

: iRow,jColumn,pulsingPercent, 
: iPapaFlag,PapaGama,PapaNu,PapaT0,PapaPeak,

: Low_frequencies_taper,

: lowFreqCoeff)

close(ioInput)

call system('EXSIM_Steve2.exe')

open(ioSim, file='simulatedParameters.out', status='unknown')

read (ioSim, $\left.{ }^{*}\right)(\operatorname{simpsa}(\mathrm{j}), \mathrm{j}=1,105)$

close(ioSim)

do $\mathrm{i}=1,105$

$\operatorname{simpsa}(\mathrm{i})=\operatorname{simpsa}(\mathrm{i}) / 981.0$

end do

do $\mathrm{i}=1,105$

psaResidual $(\mathrm{i}, 3)=\log (\mathrm{psa}(\mathrm{i}))-\log (\operatorname{simpsa}(\mathrm{i}))$

end do

$\operatorname{avgResMid}=0$

freqCount $=0$

do $\mathrm{i}=1,105$

if (freq(i).ge.lowFreqLimit.and.freq(i).le.highFreqLimit) then

avgResMid $=$ avgResMid + psaResidual $(i, 3)$

freqCount $=$ freqCount +1

end if

end do

$\operatorname{avgResMid}=\operatorname{avgResMid} /$ float(freqCount $)$

if (avgResMid.gt.0) then

lowStress $=$ midStress

do $\mathrm{i}=1,105$

$\operatorname{psaResidual}(\mathrm{i}, 1)=\operatorname{psaResidual}(\mathrm{i}, 3)$

end do

else if (avgResMid.lt.0) then

highStress $=$ midStress

do $\mathrm{i}=1,105$

psaResidual $(i, 2)=\operatorname{psaResidual}(\mathrm{i}, 3)$

end do

stressDiff $=$ midStress - pastStress

pastStress $=$ midStress

end if 
end do !bisection method loop

if (error.eq.0) then

bestStress $=$ midStress

end if

return

end

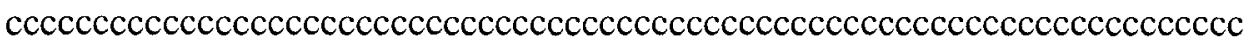

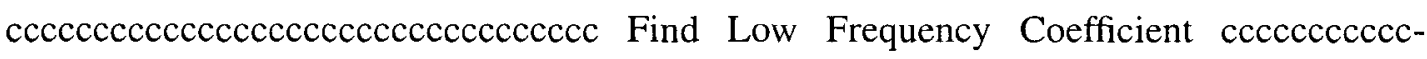
$\operatorname{ccccccccccccccc}$

function bestLFC(highLFCLimit, lowLFCLimit, lowFreqCoeff,

: lowFreqLimit, error,

: ioInput,FaultStrike,FaultDip,h,numberOfFaults,segFaultLat,

: segFaultLon,segFaultStrike, segFaultDip,segFaultLength,FaultLat,

: FaultLon,siteLat, siteLon,FaultLength, FaultWidth,dl_in, dw_in,

: nl,nw,nsubs,segnl, endOfFaultSegment,y,i0_in,j0_in,nHypocenters,

: dt, beta, rho, amag,stress,iKapa,fmax,Q0,eta, Qmin,

: rg1,rg2,pow 1,pow2,pow3,rmin,rd1,rd2,durmin,b1,b2,b3,iwind,

: flocut, nslope,nfreq,freq1,freq2,nfout, freq_out,f_stem,

: namp1,fresp1, numSiteAmp, siteAmp,iseed, nsims,damp,

: Islipweight, slipWeights,iRow,jColumn,pulsingPercent,

: iPapaFlag,PapaGama,PapaNu,PapaT0,PapaPeak,Low_frequencies_taper,

: psaResidual,ioSim,simpsa,psa,freq,stationNumber,earthID)

character InputFileName*30,f_stem $* 30$,fresp $1 * 30$,fresp $2 * 30$

character ampFile*30, siteAmp*30, flfc*30

dimension slipWeights $(200,200)$, siteLocation $(300,2)$

dimension segFaultLat(10,2), segFaultLon(10,2),segFaultStrike(10),

: segFaultDip(10),segFaultLength(10),psa(105),freq(105)

dimension dl(10), segnl(10), psaResidual(105,3), simpsa(105)

real highLFC, lowLFC, midLFC, lowFreqLimit

real avgResLow, avgResHigh, avgResMid, LFCDiff

integer error, freqCount, endOfFaultSegment(10)

real lowFreqCoeff, highLFCLimit, lowLFCLimit

real freq_out $(*)$

integer stationNumber, earthID, numAmpFile, numSiteAmp

highLFC $=$ highLFCLimit

lowLFC = lowLFCLimit 
error $=0$

c Create the name for the output files

f_stem = 'Output_000_000_00000_0000000'

write(f_stem(8:10),'(i3.3)') earthID

write(f_stem(12:14),'(i3.3)') stationNumber

write(f_stem(16:20),'(f5.1)') stress

write(f_stem(22:28),'(f7.4)') lowLFC

call trim_c(f_stem, nc_f_stem)

open (ioInput, file='EXSIM_MFIn.in',status='unknown')

call writeInputFile(ioInput,

: FaultStrike,FaultDip,h,

: numberOfFaults,

: segFaultLat, segFaultLon,

: segFaultStrike, segFaultDip,

: segFaultLength,

: FaultLat,FaultLon,

: siteLat, siteLon,

: FaultLength, FaultWidth,

: dl_in, dw_in, nl, nw, nsubs,

: segnl, endOfFaultSegment,

: $y$,

: i0_in,j0_in,nHypocenters,

: dt, beta, rho, amag,

: stress,iKapa,fmax,

: Q0,eta, Qmin,

: rg1,rg2,pow1,pow2,pow3,

: rmin,rd1,rd2,durmin,b1,b2,b3, ! trilinear duration parameters

: iwind,

: flocut, nslope,

: nfreq,freq1,freq2,

: nfout, freq_out,

: f_stem,

: namp1,fresp1, numSiteAmp, siteAmp,

: iseed, nsims,damp,

: Islipweight, slipWeights,

: iRow,jColumn,pulsingPercent,

: iPapaFlag,PapaGama,PapaNu,PapaT0,PapaPeak,

: Low_frequencies_taper,

: lowLFC) 


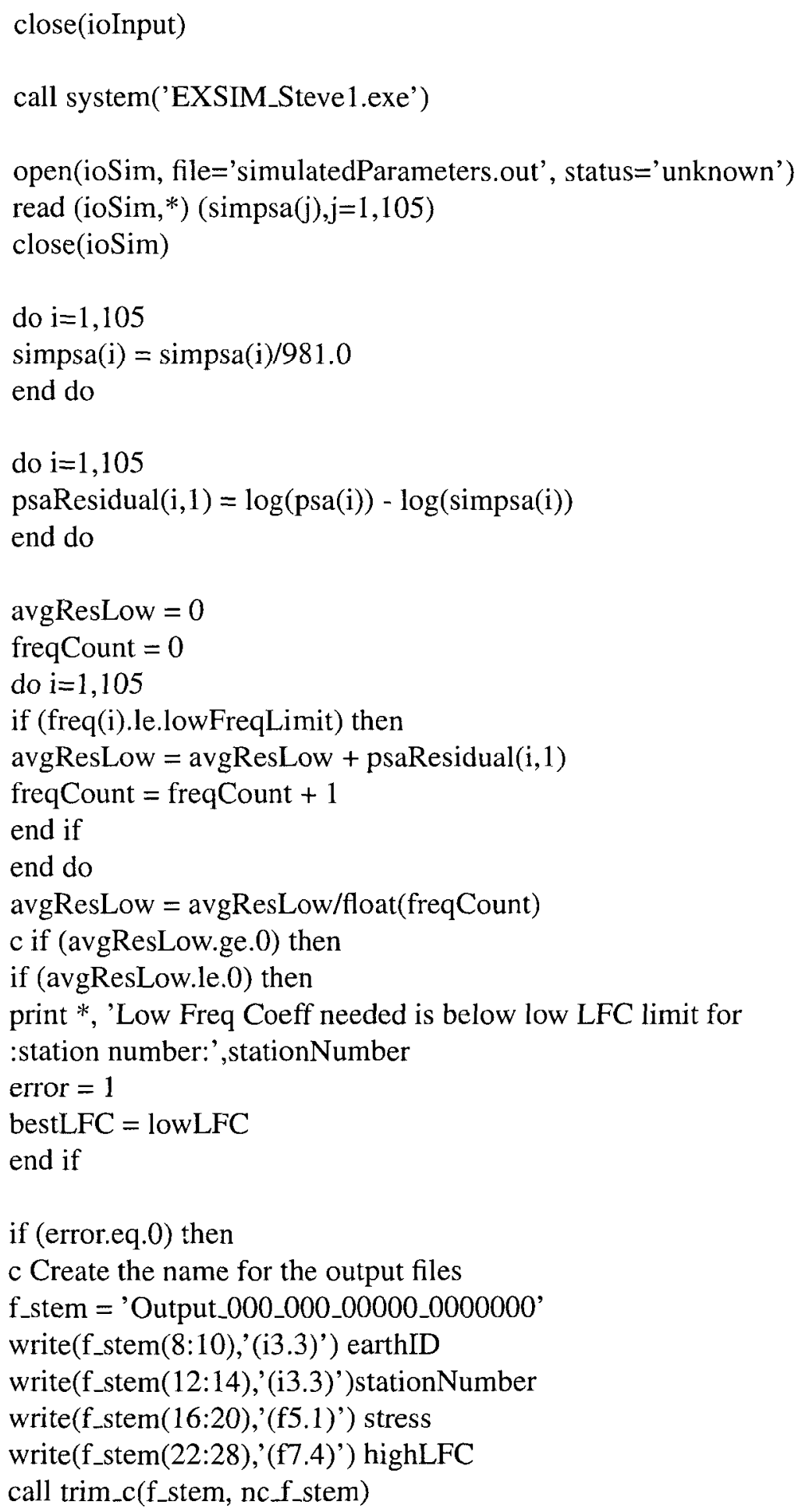


open (ioInput, file='EXSIM_MFIn.in',status='unknown')

call writeInputFile(ioInput,

: FaultStrike,FaultDip,h,

: numberOfFaults,

: segFaultLat, segFaultLon,

: segFaultStrike, segFaultDip,

: segFaultLength,

: FaultLat,FaultLon,

: siteLat, siteLon,

: FaultLength, FaultWidth,

: dl_in, dw_in, nl, nw, nsubs,

: segnl, endOfFaultSegment,

: $y$,

: i0_in,j0_in, nHypocenters,

: dt, beta, rho, amag,

: stress,iKapa,fmax,

: Q0,eta, Qmin,

: rg1,rg2,pow1,pow2,pow3,

: rmin,rd1,rd2,durmin,b1,b2,b3, ! trilinear duration parameters

: iwind,

: flocut, nslope,

: nfreq,freq1,freq2,

: nfout, freq_out,

: f_stem,

: namp1,fresp1, numSiteAmp, siteAmp,

: iseed, nsims,damp,

: Islipweight, slipWeights,

: iRow,jColumn,pulsingPercent,

: iPapaFlag,PapaGama,PapaNu,PapaT0,PapaPeak,

: Low_frequencies_taper,

: highLFC)

close(ioInput)

call system('EXSIM_Steve1.exe')

open(ioSim, file='simulatedParameters.out', status='unknown')

read $\left(\operatorname{ioSim},{ }^{*}\right)(\operatorname{simpsa}(j), j=1,105)$

close(ioSim)

do $\mathrm{i}=1,105$

$\operatorname{simpsa}(\mathrm{i})=\operatorname{simpsa}(\mathrm{i}) / 981.0$

end do 


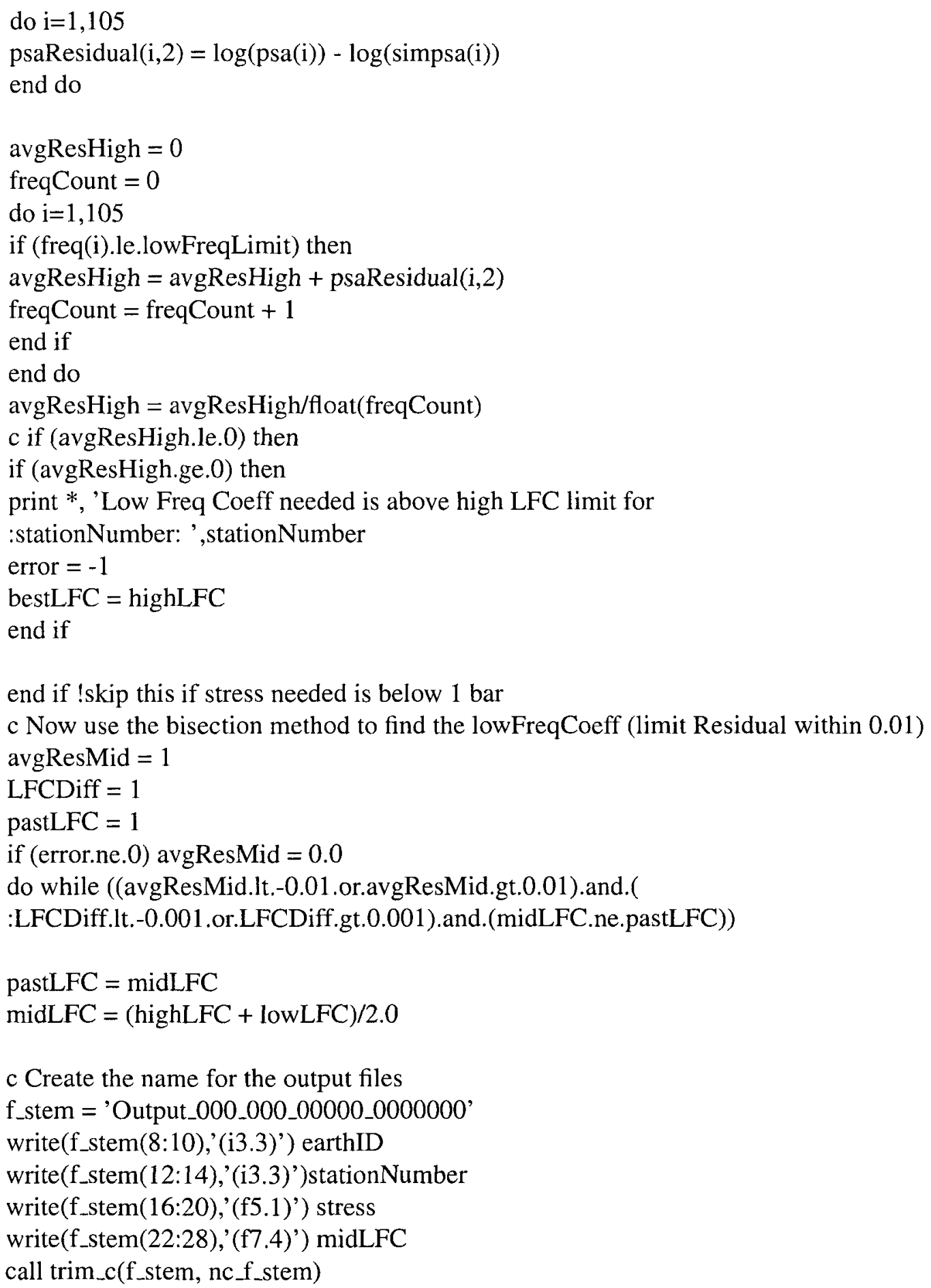


open (ioInput, file='EXSIM_MFIn.in',status='unknown')

call writeInputFile(ioInput,

: FaultStrike,FaultDip,h,

: numberOfFaults,

: segFaultLat, segFaultLon,

: segFaultStrike, segFaultDip,

: segFaultLength,

: FaultLat,FaultLon,

: siteLat, siteLon,

: FaultLength, FaultWidth,

: dl_in, dw_in, nl, nw, nsubs,

: segnl, endOfFaultSegment,

: $\mathrm{y}$

: iO_in,j0_in,nHypocenters,

: dt, beta, rho, amag,

: stress,iKapa,fmax,

: Q0,eta, Qmin,

: $r$ 1,rg2,pow1,pow2,pow3,

: rmin,rd1,rd2,durmin,b1,b2,b3, ! trilinear duration parameters

: iwind,

: flocut, nslope,

: nfreq,freq1,freq2,

: nfout, freq_out,

: f_stem,

: namp1,fresp1, numSiteAmp, siteAmp,

: iseed, nsims,damp,

: Islipweight, slipWeights,

: iRow,jColumn,pulsingPercent,

: iPapaFlag,PapaGama,PapaNu,PapaT0,PapaPeak,

: Low_frequencies_taper,

$:$ midLFC)

close(ioInput)

call system('EXSIM_Steve1.exe')

open(ioSim, file='simulatedParameters.out', status='unknown')

read (ioSim, $\left.{ }^{*}\right)$ (simpsa(j),j=1,105)

close(ioSim)

do $\mathrm{i}=1,105$

$\operatorname{simpsa}(\mathrm{i})=\operatorname{simpsa}(\mathrm{i}) / 981.0$

end do 


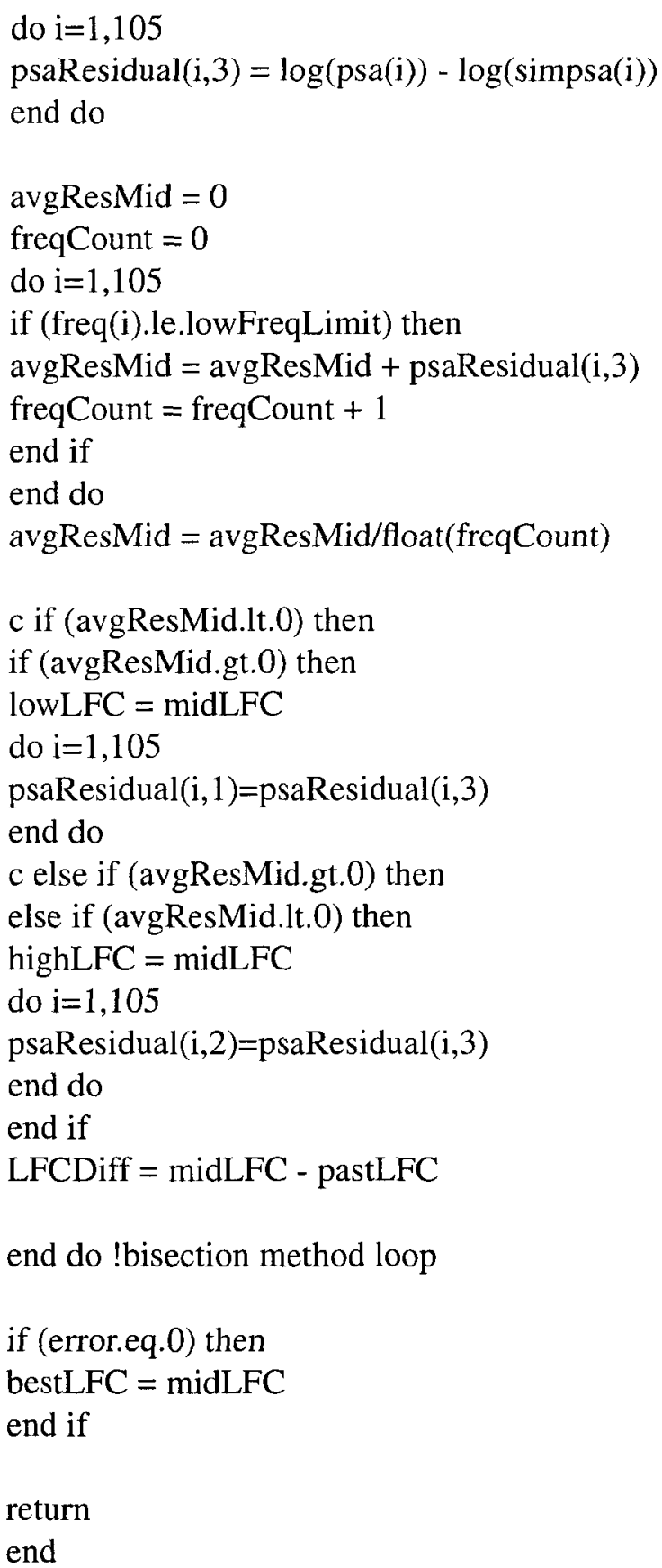

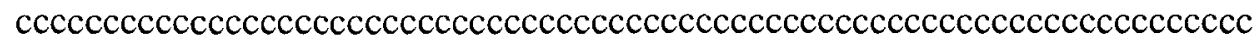

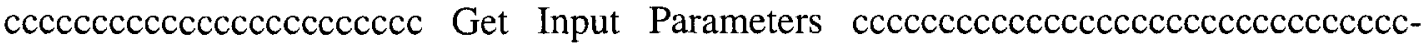
$\operatorname{cccccccccccccccccc}$ 
subroutine getInputParameters(nu_ctl,

: FaultStrike,FaultDip,h,

: numberOfFaults,

: segFaultLat, segFaultLon,

: segFaultStrike, segFaultDip,

: segFaultLength,

: FaultLat,FaultLon,

: siteLocation,numberOfSites,isitecoordflag,

: FaultLength, FaultWidth,

: dl_in, dw_in, nl, nw, nsubs,

: segnl, endOfFaultSegment,

: $y$

: i0_in,j0_in,nHypocenters,

: dt, beta, rho, amag,

: stress,iKapa,fmax,

: Q0,eta, Qmin,

: rg1,rg2,pow1,pow2,pow3,

: rmin,rd1,rd2,durmin,b1,b2,b3, ! trilinear duration parameters

: iwind,

: flocut, nslope,

: nfreq,freq1,freq2,

: nfout, freq_out,

: f_stem,

: namp1,fresp1, namp2,fresp2,

: iseed, nsims,damp,

: Islipweight, slipWeights,

: iRow,jColumn,pulsingPercent,

: iPapaFlag,PapaGama,PapaNu,PapaT0,PapaPeak,

: Low_frequencies_taper,

: D_Motazedian_Taper_coef) !Motazedian Feb, 2009

real freq_out $(*)$

real dl_in, dw_in

integer segmentNL

dimension slipWeights $(200,200)$, siteLocation $(300,2)$

dimension segFaultLat(10,2), segFaultLon(10,2), segFaultStrike(10), : segFaultDip(10),segFaultLength(10)

dimension dl(10), segnl(10)

integer endOfFaultSegment(10) 
character f_stem $*(*)$,

: fresp $1 * 30$, fresp $2 * 30$, aline $* 60$,

: version_ct $1 * 8$, version_in*30

character cmnts $2 \operatorname{skip}(50) * 80$, buf_in*10

logical f_exist

$\mathrm{pi}=4.0^{*} \operatorname{atan}(1.0)$

$\mathrm{d} 2 \mathrm{r}=\mathrm{pi} / 180.0$

version_ctl $=$, ,

version_ctl $=$ '02/10/10'

call trim_c(version_ctl,nc_version_ctl)

call skipcmnt(nu_ctl,cmnts2skip,nc_cmnts2skip)

version_in $=$, ,

read(nu_ctl,'(a)') version_in

call trim_c(version_in,nc_version_in)

if (version_ctl(1:nc_version_ctl) .ne.

: version_in(1:nc_version_in)) then

write $\left.{ }^{*}, '(a)^{\prime}\right)$

:' The control file has the wrong version number; STOP!'

close(nu_ctl)

stop

end if

call skipcmnt(nu_ctl,cmnts2skip,nc_cmnts2skip)

aline $=$, ,

read (nu_ctl,'(a)')aline

call trim_c(aline, nc_aline)

call skipcmnt(nu_ctl,cmnts2skip,nc_cmnts2skip)

read (nu_ctl,*) amag,stress,iKapa,fmax

CCCCCCCCCCCCCCCCCCCCCCCCCCCCCCCCCCCCCCCCCCCCCCCCCCCCCCCCCCCCCCCI

$\mathrm{C}$ This part reads in and calculates the things associated with the faults

call skipcmnt(nu_ctl,cmnts2skip,nc_cmnts2skip)

read (nu_ctl, ${ }^{*}$ ) numberOfFaults, FaultWidth, h

if (numberOfFaults.gt.10) then

print *, "Too many Fault Segments, must use less than 10."

stop 
end if

call skipcmnt(nu_ctl,cmnts2skip,nc_cmnts2skip)

do $\mathrm{i}=1$, numberOfFaults

read (nu_ctl, ${ }^{*}$ ) segFaultLat(i,1),segFaultLon(i,1),

: segFaultLat(i,2), segFaultLon(i,2),

: segFaultDip(i)

end do

FaultLat $=$ segFaultLat $(1,1)$

FaultLon = segFaultLon $(1,1)$

FaultDip $=$ segFaultDip $(1)$

C Calculate the fault segment lengths, the total length of the fault and the strike angles

FaultLength $=0$

FaultStrike $=$ segFaultStrike $(1)$

call skipcmnt(nu_ctl,cmnts2skip,nc_cmnts2skip)

read (nu_ctl,*) dl_in, dw_in

C Treat all fault segments as having the same width, but different lengths $\mathrm{nw}=$ FaultWidth/dw_in

if (nw .lt. 1) nw = 1

$\mathrm{dw}=$ FaultWidth/float(nw) $!$ Need to reset $\mathrm{dw}$ because nw is an integer

$\mathrm{nl}=0$

do $i=1$, numberOfFaults

segnl(i)=segFaultLength(i)/dl_in

if (segnl(i) .lt. 1) segnl(i) $=1$

$\mathrm{nl}=\mathrm{nl}+\operatorname{segnl}(\mathrm{i})$

endOfFaultSegment $(\mathrm{i})=\mathrm{nl}$

segmentNL = segnl(i) !needed dummy integer value for next line to work

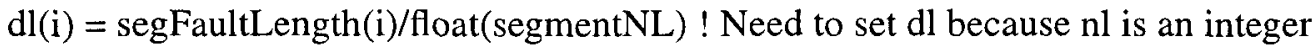
end do

C NOTE: dl is an array because it will be different for each segment!

nsubs $=\mathrm{nw} * \mathrm{nl}$ 
if(nl.gt.200.or.nw.gt.200) then

print *,"Burp!, you exceeded the

: the array dimentions, $(200,200) "$

stop

end if

call skipcmnt(nu_ctl,cmnts2skip,nc_cmnts2skip)

read (nu_ctl,*) i0_in,j0_in,nHypocenters

if (nl .eq. 1 and. nw .eq. 1 ) then ! a single subsource

i0_in $=1$

j0_in $=1$

nHypocenters $=1$

end if

call skipcmnt(nu_ctl,cmnts2skip,nc_cmnts2skip)

read (nu_ctl, ${ }^{*}$ ) beta,rho, y, dt

gsprd:

call skipcmnt(nu_ctl,cmnts2skip,nc_cmnts2skip)

read (nu_ctl, $\left.{ }^{*}\right) \operatorname{rg} 1$, rg2,pow1,pow2,pow3

Q:

call skipcmnt(nu_ctl,cmnts2skip,nc_cmnts2skip)

read (nu_ctl,*) Q0,eta, Qmin

Duration:

call skipcmnt(nu_ctl,cmnts2skip,nc_cmnts2skip)

read (nu_ctl, ${ }^{*}$ ) rmin,rd1,rd2,durmin,b1,b2,b3

call skipcmnt(nu_ctl,cmnts2skip,nc_cmnts2skip)

read (nu_ctl,*) iwind

if (iwind.eq.0) write $\left({ }^{*},{ }^{*}\right)$ "Windowing info :

* tapered boxcar"

if (iwind.eq.1) write (*,*)'Windowing info :

* Saragoni-Hart"

call skipcmnt(nu_ctl,cmnts2skip,nc_cmnts2skip)

$\operatorname{read}($ nu_ctl,*) flocut, nslope

call skipcmnt(nu_ctl,cmnts2skip,nc_cmnts2skip) 
read (nu_ctl,*) damp

call skipcmnt(nu_ctl,cmnts2skip,nc_cmnts2skip)

read $\left(n u_{-}\right.$ctl, $\left.{ }^{*}\right)$ nfreq, freq1, freq2

nfreq_max $=500$

if (iabs(nfreq).gt.nfreq_max) then

print $*$,',

print $*$, ' nfreq $=$ ', nfreq,

:' but it cannot exceed', nfreq_max

print *, STOPPING!!!'

stop

endif

call skipcmnt(nu_ctl,cmnts2skip,nc_cmnts2skip)

read $\left(\right.$ nu_ctl, $\left.{ }^{*}\right)$ nfout

call skipcmnt(nu_ctl,cmnts2skip,nc_cmnts2skip)

read(nu_ctl, ${ }^{*}$ ) (freq_out(i),i=1,nfout)

call skipcmnt(nu_ctl,cmnts2skip,nc_cmnts2skip)

$\mathrm{f}_{\mathrm{w}}$ stem $=$,

read (nu_ctl,'(a)') f_stem

call trim_c(f_stem, nc_f_stem)

call skipcmnt(nu_ctl,cmnts2skip,nc_cmnts2skip)

f_exist $=$.false.

frespl $=$, ,

read (nu_ctl,'(a)') fresp1

call trim_c(fresp1,nc_fresp1)

inquire(file=fresp $1\left(1: n c \_f r e s p 1\right)$, exist=f_exist)

if (.not. f_exist) then

print *', file '//fresp1(1:nc_fresp1)//

: ' does not exist; QUITTING!!!'

stop

else

! write(*,'(2a)') ' Input file name for crustal'//

!:' amplification :'//fresp1(1:nc_fresp1)

end if

call skipcmnt(nu_ctl,cmnts2skip,nc_cmnts2skip)

read (nu_ctl,*) namp1

! write( $\left.{ }^{*},(a, i 5)^{\prime}\right)$ ' No. of lines in the file for crustal'// 
! : ' amplification:', namp1

call skipcmnt(nu_ctl,cmnts2skip,nc_cmnts2skip)

f_exist $=$.false.

fresp2 $=$, ,

read (nu_ctl,'(a)') fresp2

call trim_c(fresp2,nc_fresp2)

inquire(file $=$ fresp2(1:nc_fresp2), exist=f_exist)

if (.not. f_exist) then

print *,' file '//fresp2(1:nc_fresp2)//

:' does not exist; QUITTING!!!'

stop

else

! write $\left.{ }^{*},(2 a)^{\prime}\right)$ ' Input file name for'//

!:' site amplification :'//fresp2(1:nc_fresp2)

end if

call skipcmnt(nu_ctl,cmnts2skip,nc_cmnts2skip)

read (nu_ctl, ${ }^{*}$ ) namp2

! write (*,'(a,i5)') ' No. of lines in the file for site'//

! : ' amplification:', namp2

call skipcmnt(nu_ctl,cmnts2skip,nc_cmnts2skip)

read(nu_ctl,*) Low_frequencies_taper, D_Motazedian_Taper_coef !Motazedian Feb,2009

if(Low_frequencies_taper.ne.0.and.Low_frequencies_taper.ne.1)then

print *, Low frequencies taper should be 0 or 1 ; QUITTING!!!'

stop

endif

c if(Low_frequencies_taper.eq.1)then !Motazedian Feb,2009

c if (D_Motazedian_Taper_coef.ge.1)then

c print *,'D_Motazedian_Taper_coef Coef should be ¡1; QUITTING!!!'

c stop

$c$ endif

$c$ endif

call skipcmnt(nu_ctl,cmnts2skip,nc_cmnts2skip)

read (nu_ctl,*)pulsingPercent

Get types of averages for FAS and PSA 
call skipcmnt(nu_ctl,cmnts2skip,nc_cmnts2skip) read (nu_ctl,*) iPapaFlag,PapaGama,PapaNu,PapaT0,PapaPeak write(*,'(a,i2,4f8.3)')'Analytical flag and its Gama,Nu,T0,peak * :", iPapaFlag,PapaGama,PapaNu,PapaT0,PapaPeak

call skipcmnt(nu_ctl,cmnts2skip,nc_cmnts2skip) read (nu_ctl, ${ }^{\text {) }}$ iseed, nsims write $\left.{ }^{*},(a, 1 x, 16,1 x, 14)^{\prime}\right)$

! : 'Iseed, No. of trials for PSA calculation: ',

$!$ : iseed, nsims

call skipcmnt(nu_ctl,cmnts2skip,nc_cmnts2skip) read (nu_ctl,*)numberOfSites, isitecoordflag ! write(*,*)"Number of Sites Around the Fault :", $! *$ numberOfSites print *, 'Type of coordinates (1=lat,long; $2=\mathrm{R}, \mathrm{Az} ; 3=\mathrm{N}, \mathrm{E})$ :', : isitecoordflag

call skipcmnt(nu_ctl,cmnts2skip,nc_cmnts2skip) do $\mathrm{i}=1$, numberOfSites read $\left(\right.$ nu_ctl, $\left.{ }^{*}\right)$ siteLocation(i,1), siteLocation(i,2) print *, SiteLat,SiteLon for Site \#', : i,' = ', siteLocation(i,1), siteLocation(i,2) enddo

call skipcmnt(nu_ctl,cmnts2skip,nc_cmnts2skip) read (nu_ctl,*) Islipweight write( ${ }^{*},(a, i 8)$ ')'SSlip flag $1=$ Random, $0=$ yours * :",Islipweight

if (Islipweight.eq.-1) then ! assign unity to all cells do $\mathrm{i}=1, \mathrm{nl}$ do $\mathrm{j}=1$, nw $\operatorname{slipWeights}(\mathrm{i}, \mathrm{j})=1.0$

end do end do else if (Islipweight.eq.0) then call skipcmnt(nu_ctl,cmnts2skip,nc_cmnts2skip) read(nu_ctl, $\left.{ }^{*}\right)((\operatorname{slip} W e i g h t s(i, j), i=1, n l), j=1, n w)$ write(*,'(/a)')" *** read slip distribution ***" else if (Islipweight.eq.1) then 
call createRandomWeight(nl,nw,slipWeights)

else

print *,' Islipweight = ', Islipweight

print *, 'NOT A LEGAL VALUE; STOPPING'

stop

endif

return

end

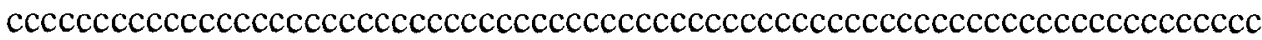

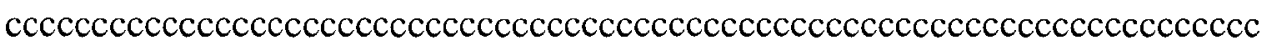

subroutine readEarthquakeStationParameters (ioStation, pga, pgv,

: pgd, NEHRP, siteLat, siteLon, lowF, psaT,

: earthID, siteAmp, moreStations, numSiteAmp,

: ioAmpFile, ioSiteAmp, stationNumber)

logical f_exist

integer moreStations

dimension psaT $(105,500)$

character NEHRP, ampFile*30, siteAmp*30

real lowF, siteLat, siteLon

integer nfreq

real freq

integer ampFileStat, siteAmpStat

integer earthID, stationNumber

dimension pga(500), pgv(500), pgd(500)

f_exist $=$ false

read (ioStation, ${ }^{*}$, iostat=moreStations) earthID, NEHRP, siteLat, :siteLon, pga(stationNumber),pgv(stationNumber),

:pgd(stationNumber), (psaT(i,stationNumber), i=1,105)

if (NEHRP.eq.' $C$ ') then

siteAmp = 'NEHRP_site_C.txt'

else if (NEHRP .eq. ' $D$ ') then

siteAmp = 'NEHRP_site_D.txt'

else if (NEHRP.eq.'B'.or.NEHRP.eq.'A') then

siteAmp = 'hard_rock.txt'

else 
siteAmp = 'siteAmplification.txt'

end if

call trim_c(siteAmp,nc_siteAmp)

inquire(file=siteAmp(1:nc_siteAmp), exist=f_exist)

if (.not. f_exist) then

print *, file '//siteAmp(1:nc_siteAmp)//

:' does not exist; QUITTING!!!'

call system('pause')

stop

end if

open (ioSiteAmp,file=siteAmp,status='unknown')

count $=0$

site AmpStat $=0$

do while (siteAmpStat.eq.0)

count $=$ count +1

read (ioSiteAmp, *, iostat=siteAmpStat) nfreq,freq

end do

numSiteAmp $=$ count -1

close (iositeAmp)

return

end

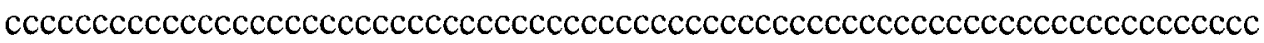

ccccccccccccccccccccccccccccccccccccccccccccccccccccccccccccccccccccc

subroutine createRandomWeight(nl,nw,slipWeights)

common $/$ seed $/$ iseed

dimension slipWeights $(200,200)$

do $\mathrm{i}=1, \mathrm{nl}$

do $\mathrm{j}=1, \mathrm{nw}$

$\mathrm{c}^{* *}$ draw random number 0 to 1 . This was changed from

$\mathrm{c}$ using $=$ ggnqf(iseed) +1 . Also impose nonzero weight.

slipWeights $(i, j)=\operatorname{Ran} 1$ (iseed)

if (slipWeights(i,j).le.0.) slipWeights $(i, j)=0.001$

enddo

enddo

return

end

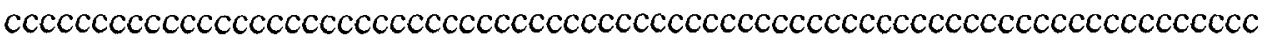


FUNCTION ran1(idum)

INTEGER idum,IA,IM,IQ,IR,NTAB,NDIV

REAL ran 1,AM,EPS, RNMX

PARAMETER $(\mathrm{IA}=16807, \mathrm{IM}=2147483647, \mathrm{AM}=1 . / \mathrm{IM}, \mathrm{IQ}=127773, \mathrm{IR}=2836$,

*NTAB=32,NDIV=1+(IM-1)/NTAB,EPS=1.2e-7,RNMX=1.-EPS)

INTEGER j,k,iv(NTAB),iy

SAVE iv,iy

DATA iv /NTAB $* 0 /$, iy /0/

if (idum.le.0.or.iy.eq.0) then

idum $=\max ($-idum, 1$)$

do $11 \mathrm{j}=\mathrm{NTAB}+8,1,-1$

$\mathrm{k}=\mathrm{idum} / \mathrm{IQ}$

idum $=I A *($ idum $-k * I Q)-I R * k$

if (idum.lt.0) idum=idum+IM

if $(\mathrm{j} . l \mathrm{l} . \mathrm{NTAB}) \mathrm{iv}(\mathrm{j})=\mathrm{idum}$

11 continue

$i y=i v(1)$

endif

$\mathrm{k}=\mathrm{idum} / \mathrm{IQ}$

idum $=I A *$ (idum- $k * I Q)-I R * k$

if (idum.It.0) idum=idum+IM

$\mathrm{j}=1+\mathrm{iy} / \mathrm{NDIV}$

$i y=i v(j)$

iv $(j)=i d u m$

$\operatorname{ran} 1=\min \left(A^{*} * \mathrm{iy}, \mathrm{RNMX}\right)$

return

END

C (C) Copr. 1986-92 Numerical Recipes Software !6)-"11j.

END RAN1

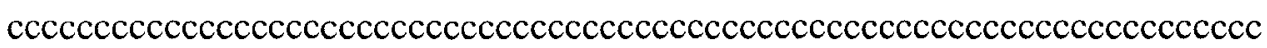

subroutine trim_c(cstr, nchar)

strips leading and trailing blanks from cstr, returning the

result in cstr, which is now nchar characters in length

Here is a sample use in constructing a column header, filled out with periods: 
* Read idtag:

idtag $=$, ,

read(nu_in, '(1x,a)') idtag

call trim_c(idtag, nc_id)

* Set up the column headings:

colhead $=$, '

colhead $=\operatorname{idtag}(1:$ nc_id $) / /$ '.....' $!$ nc_id $+6 i$ length of colhead

Dates: $12 / 23 / 97^{\prime}$ - written by D. Boore

$12 / 08 / 00$ - pad with trailing blanks. Otherwise some comparisons

of the trimmed character string with other strings

can be in error because the original characters are left

behind after shifting. For example, here is a string

before and after shifting, using the old version:

col:12345

MTWH before

MTWHH after (but nc $=4$ ).

03/21/01 - Check for a zero length input string

$11 / 09 / 01$ - Change check for zero length string to check for all blanks

character $\left.\operatorname{cstr} *^{*}\right)$

if(cstr .eq. " ') then

nchar $=0$

return

end if

nend $=\operatorname{len}(\mathrm{cstr})$

if(nend .eq. 0) then

nchar $=0$

return

end if

do $\mathrm{i}=$ nend, $1,-1$

if (cstr(i:i) .ne. ',) then

nchar $2=\mathrm{i}$

goto 10

end if

end do

10 continue 
do $\mathrm{j}=1$, nchar 2

if $(\operatorname{cstr}(j: j)$.ne. ', $)$ then

nchar $1=\mathrm{j}$

goto 20

end if

end do

20 continue

nchar $=$ nchar $2-$ nchar $1+1$

$\operatorname{cstr}(1:$ nchar $)=\operatorname{cstr}($ nchar $1:$ nchar 2$)$

if (nchar .lt. nend) then

do $i=$ nchar +1 , nend

$\operatorname{cstr}(\mathrm{i}: \mathrm{i})=$, ,

end do

end if

return

end

skipcmnt

subroutine skipcmnt(nu, comment, ncomments)

Skip text comments in the file attached to unit nu, but save skipped comments in character array comment. Skip at least one line, and more as long as the lines are preceded by "_-" or "!".

Dates: 04/16/01 - Written by D. Boore

12/07/01 - Added check for eof

$11 / 04 / 03$ - Use trim_c to trim possible leading blank

02/03/07 - Initialize comments to blank

character comment $(*)^{*}(*)$, buf*80

ncomments $=0$

100 buf $=$, ,

read (nu,'(a)',end=999) buf

call trim_c(buf,nc_buf)

if (buf(1:1) .eq.'!' .or. buf(1:1) .eq.'-' .or.

: ncomments +1 .eq. 1$)$ then

ncomments $=$ ncomments +1 


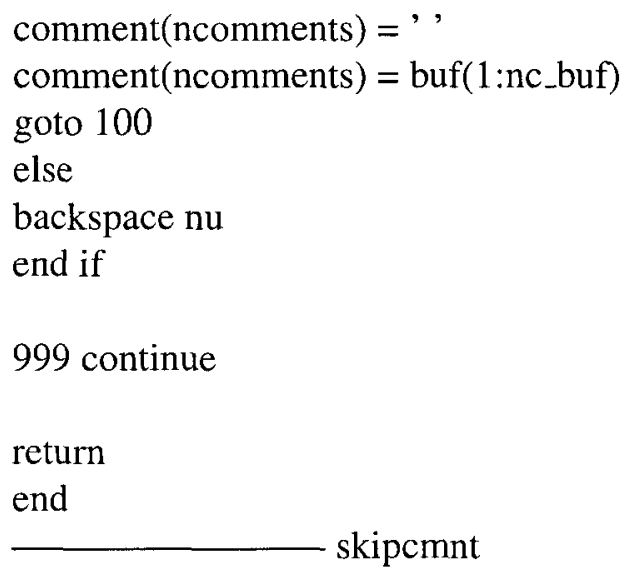

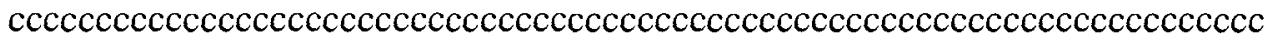
real function round ( $\mathrm{x}$, places)

real $x$

integer places

real tempx, power

if (places .ge. 0 ) then

power $=10^{* *}$ places

tempx $=\mathrm{x}^{*}$ power

round $=\operatorname{nint}($ tempx $) /$ power

else

print *, 'negative arguement for places - no rounding done'

round $=\mathrm{x}$

end if

return

end

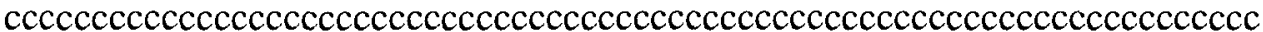

BEGIN TIME_DIFF

subroutine time_diff(time_start, time_stop, time_elapsed) 
Dates: 02/18/09 - Written by D.M. Boore

To be used with

Standard Fortran 90 intrinsic Subroutine DATE_AND_TIME

character dat $x * 8$, timx $* 10$

call DATE_AND_TIME( datx, timx )

Date is returned as 'CCYYMMDD'

Time is returned as 'hhmmss.sss'

character time_start*(*), time_stop* $\left(^{*}\right)$

read(time_start(1:10),'(i2,i2,f6.3)')

: ihb, imb, secb

read(time_stop(1:10),'(i2,i2,f6.3)')

: ihe, ime, sece

time_elapsed $=$

$: 3600.0 *$ float $($ ihe-ihb $)+60.0 *$ float $($ ime-imb $)+$ sece-secb

return

end

END TIME_DIFF

cccccccccccccccccccccccccccccccccccccccccccccccccccccccccccccccccccccc subroutine getFreq(nfreq,freq)

$\mathrm{c}$ this subroutine gets the NGA frequencies for interpetation

dimension freq(500)

nfreq $=105$

freq $(1)=1 . / 10.0$

freq $(2)=1.19 .5$

freq $(3)=1.19 .0$

freq $(4)=1 . / 8.5$

freq $(5)=1 . / 8.0$

freq $(6)=1 . / 7.5$

freq $(7)=1 . / 7.0$

freq $(8)=1 . / 6.5$

freq $(9)=1 . / 6.0$

freq $(10)=1 . / 5.5$

freq $(11)=1 . / 5.0$

freq $(12)=1 . / 4.8$

freq $(13)=1 . / 4.6$

freq $(14)=1 . / 4.4$ 


$$
\begin{aligned}
& \text { freq }(15)=1 . / 4.2 \\
& \text { freq }(16)=1 . / 4.0 \\
& \text { freq }(17)=1 . / 3.8 \\
& \text { freq }(18)=1 . / 3.6 \\
& \text { freq }(19)=1 . / 3.5 \\
& \text { freq }(20)=1 . / 3.4 \\
& \text { freq }(21)=1 . / 3.2 \\
& \text { freq }(22)=1 . / 3.0 \\
& \text { freq }(23)=1 . / 2.8 \\
& \text { freq }(24)=1 . / 2.6 \\
& \operatorname{freq}(25)=1 . / 2.5 \\
& \text { freq }(26)=1 . / 2.4 \\
& \text { freq }(27)=1 . / 2.2 \\
& \text { freq }(28)=1 . / 2.0 \\
& \text { freq }(29)=1 . / 1.9 \\
& \text { freq }(30)=1 . / 1.8 \\
& \text { freq }(31)=1 . / 1.7 \\
& \text { freq }(32)=1 . / 1.6 \\
& \text { freq }(33)=1 . / 1.5 \\
& \text { freq }(34)=1 . / 1.4 \\
& \text { freq }(35)=1 . / 1.3 \\
& \text { freq }(36)=1 . / 1.2 \\
& \text { freq }(37)=1 . / 1.1 \\
& \text { freq }(38)=1 . / 1.0 \\
& \operatorname{freq}(39)=1 . / 0.95 \\
& \text { freq }(40)=1 . / 0.9 \\
& \text { freq }(41)=1 . / 0.85 \\
& \text { freq }(42)=1 . / 0.8 \\
& \text { freq }(43)=1 . / 0.75 \\
& \text { freq }(44)=1 . / 0.7 \\
& \text { freq }(45)=1 . / 0.667 \\
& \text { freq }(46)=1 . / 0.65 \\
& \text { freq }(47)=1 . / 0.6 \\
& \text { freq }(48)=1 . / 0.55 \\
& \text { freq }(49)=1 . / 0.5 \\
& \text { freq }(50)=1 . / 0.48 \\
& \text { freq }(51)=1 . / 0.46 \\
& \text { freq }(52)=1 . / 0.45 \\
& \operatorname{freq}(53)=1 . / 0.44 \\
& \operatorname{freq}(54)=1 . / 0.42 \\
& \text { freq }(55)=1 . / 0.4 \\
& \text { freq }(56)=1 . / 0.38 \\
& \text { freq }(57)=1 . / 0.36
\end{aligned}
$$




$$
\begin{aligned}
& \text { freq }(58)=1 . / 0.35 \\
& \operatorname{freq}(59)=1 . / 0.34 \\
& \text { freq }(60)=1 . / 0.32 \\
& \text { freq }(61)=1 . / 0.3 \\
& \text { freq }(62)=1 . / 0.29 \\
& \text { freq }(63)=1 . / 0.28 \\
& \text { freq }(64)=1 . / 0.26 \\
& \text { freq }(65)=1 . / 0.25 \\
& \text { freq }(66)=1 . / 0.24 \\
& \text { freq }(67)=1 . / 0.22 \\
& \text { freq }(68)=1 . / 0.2 \\
& \text { freq }(69)=1 . / 0.19 \\
& \text { freq }(70)=1 . / 0.18 \\
& \text { freq }(71)=1 . / 0.17 \\
& \text { freq }(72)=1 . / 0.16 \\
& \text { freq }(73)=1 . / 0.15 \\
& \text { freq }(74)=1 . / 0.14 \\
& \text { freq }(75)=1 . / 0.133 \\
& \text { freq }(76)=1 . / 0.13 \\
& \text { freq }(77)=1 . / 0.12 \\
& \text { freq }(78)=1 . / 0.11 \\
& \text { freq }(79)=1 . / 0.1 \\
& \text { freq }(80)=1 . / 0.095 \\
& \text { freq }(81)=1 . / 0.09 \\
& \text { freq }(82)=1 . / 0.085 \\
& \text { freq }(83)=1 . / 0.08 \\
& \text { freq }(84)=1 . / 0.075 \\
& \text { freq }(85)=1 . / 0.07 \\
& \text { freq }(86)=1 . / 0.067 \\
& \text { freq }(87)=1 . / 0.065 \\
& \text { freq }(88)=1 . / 0.06 \\
& \text { freq }(89)=1 . / 0.055 \\
& \text { freq }(90)=1 . / 0.05 \\
& \text { freq }(91)=1 . / 0.048 \\
& \text { freq }(92)=1 . / 0.046 \\
& \text { freq }(93)=1 . / 0.045 \\
& \text { freq }(94)=1 . / 0.044 \\
& \text { freq }(95)=1 . / 0.042 \\
& \text { freq }(96)=1 . / 0.040 \\
& \text { freq }(97)=1 . / 0.036 \\
& \text { freq }(98)=1 . / 0.035 \\
& \text { freq }(99)=1 . / 0.032 \\
& \text { freq }(100)=1 . / 0.03
\end{aligned}
$$




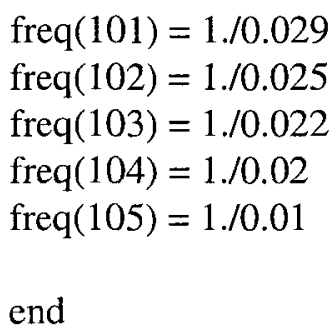

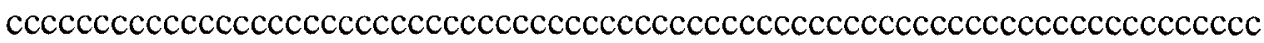

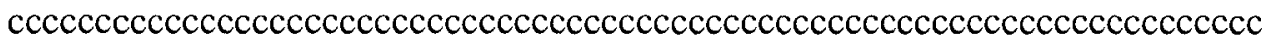
subroutine getFreqPSA(psaT,psa,j)

$\mathrm{c}$ this converts the psa from period to frequency dimension psa(105),psaT(105,500)

do $\mathrm{i}=1,105$

$\operatorname{psa}(i)=\operatorname{psaT}((106-i), j)$ end do

end

ccccccccccccccccccccccccccccccccccccccccccccccccccccccccccccccccccccccc 


\section{List of References}

[1] Aki, K. (1967), Scaling law of seismic spectrum, J. Geophys. Res., 72, 1217-1231.

[2] Anderson, J. G., and S. E. Hough (1984), A model for the shape of the fourier amplitude spectrum of acceleration at high frequencies, Bull. Seism. Soc. Am., 74, 19691993.

[3] Atkinson, G. M. (1995), Attenuation and source parameters of earthquakes in the Cascadia region, Bull. Seism. Soc. Am., 85, 1327-1342.

[4] Atkinson, G. M., and D. M. Boore (1995), Ground-motion relations for eastern North America, Bull. Seism. Soc. Am., 85, 17-30.

[5] Atkinson, G. M., and D. M. Boore (1998), Evaluation of models for earthquake source spectra in eastern North America, Bull. Seism. Soc. Am., 88, 917-934.

[6] Atkinson, G. M., and D. M. Boore (2003), Empirical ground-motion relations for subduction-zone earthquakes and their application to the Cascadia and other regions, Bull. Seism. Soc. Am., 93, 1703-1729.

[7] Atkinson, G. M., and D. M. Boore (2006), Earthquake ground-motion prediction equations for eastern North America, Bull. Seism. Soc. Am., 96, 2181-2205.

[8] Atkinson, G. M., and W. Silva (1997), An empirical study of earthquake source spectra for California earthquakes, Bull. Seism. Soc. Am., 87, 97-113.

[9] Atkinson, G. M., and W. Silva (2000), Stochastic modeling of California ground motions, Bull. Seism. Soc. Am., 90, 255-274.

[10] Atkinson, G. M., K. Assatourians, D. M. Boore, K. Campbell, and D. Motazedian (2009), A guide to differences between stochastic point-source and stochastic finitefault simulations, Bull. Seism. Soc. Am., 99, 3192-3201.

[11] Benz, H. M., A. Frankel, and D. M. Boore (1997), Regional Lg attenuation for the continental United States, Bull. Seism. Soc. Am., 87, 606-619. 
[12] Beresnev, I. A., and G. M. Atkinson (1997), Modeling finite-fault radiation from the $\omega^{n}$ spectrum, Bull. Seism. Soc. Am., 87, 67-84.

[13] Beresnev, I. A., and G. M. Atkinson (1998a), Finsim: A fortran program for simulating stochastic acceleration time histories from finite faults, Seism. Res. Lett., 69, $27-32$.

[14] Beresnev, I. A., and G. M. Atkinson (1998b), Stochastic finite-fault modeling of ground motions from the 1994 Northridge, California, earthquake. I. Validation on rock sites, Bull. Seism. Soc. Am., 88, 1392-1401.

[15] Beresnev, I. A., and G. M. Atkinson (2002), Source parameters of earthquakes in eastern and western North America based on finite-fault modeling, Bull. Seism. Soc. Am., 92, 695-710.

[16] Bernard, P., A. Herrero, and C. Berge (1996), Modeling directivity of heterogeneous earthquake ruptures, Bull. Seism. Soc. Am., 86, 1149-1160.

[17] Boatwright, J. (1982), A dynamic model for far-field acceleration, Bull. Seism. Soc. Am., 72, 1049-1068.

[18] Boore, D. M. (1983), Stochastic simulation of high-frequency ground motions based on seismological models of the radiated spectra, Bull. Seism. Soc. Am., 73, 18651894.

[19] Boore, D. M. (1996), SMSIM: Fortran programs for simulating ground motions from earthquakes, U.S. Geol. Surv. Open-File Rept., 96-80-A.

[20] Boore, D. M. (2003), Simulation of ground motion using the stochastic method, Pure and Applied Geophysics, 160, 635-676.

[21] Boore, D. M. (2009), Comparing stochastic point-source and finite-source groundmotion simulations: SMSIM and EXSIM, Bull. Seism. Soc. Am., 99, 3202-3216.

[22] Boore, D. M., and J. Boatwright (1984), Average body-wave radiation coefficients, Bull. Seism. Soc. Am., 74, 1615-1621.

[23] Boore, D. M., and W. B. Joyner (1997), Site amplifications for generic rock sites, Bull. Seism. Soc. Am., 87, 327-341.

[24] Boore, D. M., W. B. Joyner, and L. Wennerberg (1992), Fitting the stochastic $\omega^{-2}$ source model to the observed response spectra in western North America: trade-offs between $\Delta \sigma$ and $\kappa$, Bull. Seism. Soc. Am., 82, 1956-1963. 
[25] Boore, D. M., W. B. Joyner, and T. E. Fumal (1993), Estimation of response spectra and peak accelerations from western North American earthquakes: An interim report, U.S. Geol. Surv. Open-File Rept., 93-509.

[26] Boore, D. M., W. B. Joyner, and T. E. Fumal (1997), Equations for estimating horizontal response spectra and peak acceleration from western North American earthquakes: a summary of recent work., Seism. Res. Lett., 68, 128-153.

[27] Boore, D. M., J. F. Gibbs, W. B. Joyner, J. C. Tinsley, and D. J. Ponti (2003), Estimated ground motion from the 1994 Northridge, California, earthquake at the site of the Interstate 10 and La Cienega Boulevard Bridge collaspe, West Los Angeles, California, Bull. Seism. Soc. Am., 93, 2737-2751.

[28] Boore, D. M., K. W. Campbell, and G. M. Atkinson (2010), Determination of stress parameters for eight well-recorded earthquakes in eastern North America, Bull. Seism. Soc. Am., 100, 1632-1645.

[29] Brace, W. F., and T. D. Byerlee (1966), Stick-slip as a mechanism for earthquakes, Science, 153, 990-992.

[30] Brune, J. (1970), Tectonic stress and the spectra of seismic shear waves from earthquakes, J. Geophys. Res., 75, 4997-5009.

[31] Brune, J. (1971), Correction, J. Geophys. Res., 76, 5002.

[32] Fatehi, A., and R. B. Herrmann (2008), High-frequency ground-motion scaling in the Pacific Northwest and in northern and central California, Bull. Seism. Soc. Am., 98, 709-721.

[33] Frankel, A. (1995a), Mapping seismic hazard in the central and eastern United States, Seism. Res. Lett., 66, 8-21.

[34] Frankel, A. (1995b), Simulating strong motions of large earthquakes using recordings of small earthquakes: the Loma Prieta mainshock as a test case, Bull. Seism. Soc. Am., $85,1144-1160$.

[35] Frankel, A. (2009), A constant stress-drop model for producing broadband synthetic seismograms: Comparison with the next generation attenuation relations, Bull. Seism. Soc. Am., 99, 664-680.

[36] Frankel, A. D., C. S. Mueller, T. Barnhard, D. Perkins, E. LeyenDecker, N. Dickman, S. Hanson, and M. Hopper (1996), National seismic hazard maps: Documation june 1996, U.S. Geol. Surv. Open-File Rept., 96-532. 
[37] Frankel, A. D., et al. (2002), Documation for the 2002 update of the National Seismic Hazard Maps, U.S. Geol. Surv. Open-File Rept., 02-420.

[38] Haddon, R. (1996), Earthquake source spectra in eastern North America, Bull. Seism. Soc. Am., 86, 1300-1313.

[39] Hanks, T. C. (1979), $b$ values and $\omega^{-\gamma}$ seismic source models: implications for tectonic stress variations along active crustal fault zones and the estimation of highfrequency strong ground motion, J. Geophys. Res., 84, 2235-2242.

[40] Hanks, T. C. (1982), $f_{\max }$, Bull. Seism. Soc. Am., 72, 1867-1879.

[41] Hanks, T. C., and H. Kanamori (1981), A moment magnitude scale, J. Geophys. Res., $84,2348-2350$.

[42] Hanks, T. C., and R. K. McGuire (1981), The character of high-frequency strong ground motion, Bull. Seism. Soc. Am., 71, 2071-2095.

[43] Harmsen, S. (1997), Estimating the dimunution of shear-wave amplitude with distance: application to Los Angeles, california, urban area, Bull. Seism. Soc. Am., 87, 888-903.

[44] Joyner, W. B. (1984), A scaling law for the spectra of large earthquakes, Bull. Seism. Soc. Am., 74, 1167-1188.

[45] Joyner, W. B., and D. M. Boore (1986), On simulating large earthquakes by green's functions addition of smaller earthquakes, in Earthquake Source Mechanics, American Geophysical Monograph, 37, 269-274.

[46] Kamae, K., K. Irikura, and A. Pitarka (1998), A technique for simulating strong ground motion using hybrid green's function, Bull. Seism. Soc. Am., 88, 357-367.

[47] Kanamori, H. (1977), The energy release in great earthquakes, J. Geophys. Res., 82, 2981-2987.

[48] Kanamori, H., and D. L. Anderson (1975), Theoretical basis of some empirical relations in seismology, Bull. Seism. Soc. Am., 65, 1073-1095.

[49] King, C.-Y., and L. Knopoff (1968), Stress drop in earthquakes, Bull. Seism. Soc. Am., $58,249-257$.

[50] McGuire, R. K., and T. C. Hanks (1980), RMS accelerations and spectral amplitudes of strong ground motion motion during the San Fernando, California earthquake, Bull. Seism. Soc. Am., 70, 1907-1919. 
[51] Moghaddam, H., N. Fanaie, and D. Motazedian (2010), Estimation of stress drop for some large shallow earthquakes using stochastic point source and finite fault modeling, Scientia Iranica Journal, 17, 217-235.

[52] Motazedian, D. (2006), Region-specific key seismic parameters for earthquakes in northern Iran, Bull. Seism. Soc. Am., 99, 1383-1395.

[53] Motazedian, D., and G. M. Atkinson (2005), Stochastic finite-fault modeling based on a dynamic corner frequency, Bull. Seism. Soc. Am., 95, 995-1010.

[54] Motazedian, D., and A. Monifar (2006), Hybrid stochastic finite fault modelling of 2003, M6.5, Bam earthquake, Journal of Seismology, 10, 91-103.

[55] Raoof, M., R. B. Herrmann, and L. Malagnini (1999), Attenuation and excitation of three-component ground motion in southern California, Bull. Seism. Soc. Am., 89, $888-902$.

[56] Sadigh, K., C. Y. Chang, J. A. Egan, F. Makdisi, and R. R. Youngs (1997), Attenuation relationships for shallow crustal earthquakes based on California strong motion data, Seism. Res. Lett., 68, 180-189.

[57] Safak, E., and D. M. Boore (1988), On low-frequency errors of uniformaly modulated filtered white-noise models for ground motions, Earthq. Eng. Strruct. Dyn., 16, 381388.

[58] Saragoni, R., and G. C. Hart (1974), Simulation of artifical earthquakes, Earthquake Engineering and Structial Dynamics, 2, 249-267.

[59] Sommerville, P., N. Smith, R. Graves, and N. Abrahamson (1997), Modification of empirical strong ground motion attenuation relations to include the amplitude and duration effects of rupture directivity, Seism. Res. Lett., 68, 199-222.

[60] Wells, D., and K. Coppersmith (1994), New empirical relationships among magnitude, rupture lenght, rupture width, rupture area, and surface displacement, Bull. Seism. Soc. Am., 84, 974-1002. 